\title{
Regioselectivity of Birch Reductive Alkylation of Biaryls
}

\author{
Raphaël LEBEUF, Frédéric ROBERT and Yannick LANDAIS*
}

Université Bordeaux I, Laboratoire de Chimie Organique et Organométallique, 351, Cours de la Libération, F-33405 Talence cedex, France y.landais@lcoo.u-bordeaux1.fr

\section{Supporting information}

\section{List of contents}

A) General $\quad$ S2

B) Experimental procedures for Biaryls synthesis $\quad$ S2

C) General procedure for Birch Reductive Alkylation S6

D) Experimental procedures for crinine analog synthesis $\quad \mathrm{S11}$

$\begin{array}{lr}\text { E) NMR Spectra } & \text { S14 }\end{array}$ 


\section{A) General:}

${ }^{1} \mathrm{H}$ NMR and ${ }^{13} \mathrm{C}$ NMR spectra were recorded on a Brüker Avance $300\left({ }^{1} \mathrm{H}: 300 \mathrm{MHz},{ }^{13} \mathrm{C}: 75.5\right.$ $\mathrm{MHz})$ or a Brüker AC-250 FT $\left({ }^{1} \mathrm{H}: 250 \mathrm{MHz},{ }^{13} \mathrm{C}\right.$ : $\left.62.9 \mathrm{MHz}\right)$, using $\mathrm{CDCl}_{3}$ as internal reference. The chemical shifts $(\delta)$ and coupling constants $(J)$ are expressed in ppm and hertz respectively. IR spectra were recorded on a Perkin-Elmer Paragon 1000 FT-IR spectrophotometer. HRMS were recorded on a Varian MAT 311 aparatus (for EI) or a Micromass ZABSpec TOF aparatus (for ESI). Melting points were determined by using a Büchi Totolli apparatus and are uncorrected. Merk silica gel $(0.043-0.063 \mathrm{~mm})$ was used for flash chromatography. All reactions were carried out under nitrogen. $\mathrm{CH}_{2} \mathrm{Cl}_{2}$ was distilled over $\mathrm{CaH}_{2}$. THF and $\mathrm{Et}_{2} \mathrm{O}$ were distilled from sodium and benzophenone. Acetone was distilled over calcium sulfate. $\mathrm{MeOH}$ was distilled over magnesium. $\mathrm{NH}_{3}$ gas was dried by passing through potassium hydroxide pellets. Methyl chloroacetate and chloroacetonitrile were distilled before use.

3-Hydroxy-biphenyl (5), 3-methoxy-biphenyl (7) and all other reagents and starting materials were directly used as obtained commercially. 3,5-Iododimethoxybenzene was prepared from commercial 3,5-dimethoxyaniline by a Sandmeyer reaction. ${ }^{1}$ 3,5-Dimethoxy-biphenyl (9) ${ }^{2}$, 4-(2-hydroxyphenyl)-3-buten-2-one ${ }^{3}$, 4-(3-hydroxy-phenyl)-3-buten-2-one. ${ }^{4}$ were prepared according to published procedures. 4-(4-Hydroxy-3-methoxy-phenyl)-3-buten-2-one (Vanillylidine acetone), 4(2-hydroxy-3-methoxy-phenyl)-3-buten-2-one were prepared according to a published procedure ${ }^{5}$.

\section{B) Experimental procedures for Biaryls synthesis:}

\section{General procedure for 3,5-dimethoxy substituted Biaryls: $^{6}$}

To a dry $250 \mathrm{~mL}$ two-necked flask equipped with a condenser and filled with dry methanol (60 mL) was added, at $0{ }^{\circ} \mathrm{C}$, sodium wire (690 mg, $\left.30 \mathrm{mmol}\right)$. After all sodium has been dissolved, dimethyl malonate ( $3.96 \mathrm{~g}, 30 \mathrm{mmol}$ ) was introduced and a novel portion of sodium wire (690 mg, $30 \mathrm{mmol}$ ) was added. After its dissolution, a substituted 4-(aryl)-3-buten-2-one (30 mmol) was added and the solution was refluxed for 4 hours. The mixture was diluted with water $(60 \mathrm{~mL})$ and the total volume was reduced to half on evaporator. An aqueous solution of sodium hydroxide (4.8 g, 120 mmol in $60 \mathrm{~mL} \mathrm{H}_{2} \mathrm{O}$ ) was then added and the solution was refluxed again for one hour. Concentrated $\mathrm{HCl}$ was added dropwise until the violent $\mathrm{CO}_{2}$ release stopped and discoloration of the media occurred. The flask was then cooled to $0^{\circ} \mathrm{C}$ and an insoluble brown paste was recovered by decantation, washed with cold water and dissolved in ethanol. After removal of the solvent, the crude mixture was refluxed in methanol $(66 \mathrm{~mL})$ with iodine $(16.7 \mathrm{~g}, 66 \mathrm{mmol})$ for three hours. An aqueous sodium thiosulfate solution was added and the aqueous layer was extracted with ether. The organic layer was washed with brine, dried over sodium sulfate and evaporated. Pure compounds

\footnotetext{
1 a) M. R. Pavia, M. P. Cohen, G. J. Dilley, G. R. Dubuc, T. L. Durgin, F. W. Forman, M. E. Hediger, G. Milot, T. S. Powers, I. Sucholeiki, S. L. Zhou, and D. G. Hangauer, Bioorg. Med. Chem. 1996, 4, 659-666. b) Deboves, H. J. C.; Montalbetti, C. A. G. N.; Jackson, R. F. W. J. Chem. Soc., Perkin Trans. 1 2001, 1876-1884.

2 Dol, G. C.; Kamer, C. J.; Piet van Leeuwen, W. N. M. Eur. J. Org. Chem. 1998, 359-364. Percec, V.; Golding, G. M.; Smidrkal, J.; Weichold O. J. Org. Chem. 2004, 69, 3447-3452.

${ }^{3}$ Finkelstein, B. L.; Benner, E. A.; Hendrixson, M. C.; Kranis, K. T.; Rauh, J. J.; Sethuraman, M. R.; McCann, S. F. Bioorg. Med. Chem. 2002, 10, 599-613.

4 Uchiyama, K.; Ono, A.; Hayashi, Y.; Narasaka, K. Bull. Chem. Soc. Jap. 1998, 71, 2945-2955.

5 Ducki, S.; Hadfield, J. A.; Hepworth, L. A.; Lawrence, N. J.; Liu, C. Y.; McGown, A. T. Bioorg. Med. Chem. Lett. 1997, 7, 3091-3094.

6 Dienone were prepared following a modified procedure: Tamura, Y.; Yoshimoto, Y.; Kunimoto, K.; Tada, S.; Tomita, T.; Wada, T.; Seto, E.; Murayama, M.; Shibata, Y.; Nomura, A.; Ohata, K. J. Med. Chem. 1977, 20, 709-714. 3,5-dimethoxy-phenyl were prepared following a modified procedure: A. S. Kotnis Tetrahedron Letters 1991, 32, 3441-3444. (trusting text but not the misdrawn structures in table 1)
} 
were obtained after purification by silica gel flash chromatography (petroleum ether/EtOAc mixtures).

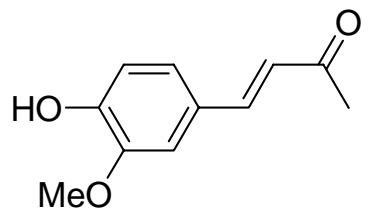

1) Dimethylmalonate, $\mathrm{Na}, \mathrm{MeOH}$ reflux, $4 \mathrm{~h}$

2) aq $\mathrm{NaOH}$, reflux, $1 \mathrm{~h}$

3) conc. $\mathrm{HCl}, 0^{\circ} \mathrm{C}$

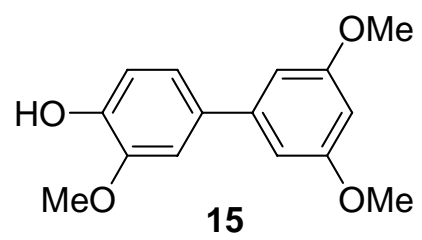

3,3',5'-Trimethoxy-biphenyl-4-ol (15). This product was obtained as a pale yellow solid (3.45 g, $13.26 \mathrm{mmol}, 44 \%)$ from 4-(4-hydroxy-3-methoxy-phenyl)-3-buten-2-one. This product could be recrystallized in DCM/petroleum ether. - m.p. 73.2-74.4 ${ }^{\circ} \mathrm{C}$. - IR (solid, $\mathrm{KBr}$ ): $v=3398 \mathrm{~cm}^{-1}, 2941$, 1603, 1523, 1445, 1251, 1209, 1150, 1064, 1022, 820, 617. - ${ }^{1} \mathrm{H}$ NMR $\left(\mathrm{CDCl}_{3}, 250 \mathrm{MHz}\right): \delta=$ 7.11-7.06 (m, 2H, aromatic H), $6.98(\mathrm{~d}, J=7.9 \mathrm{~Hz}, 1 \mathrm{H}$, aromatic $\mathrm{H}), 6.68(\mathrm{~d}, J=2.1 \mathrm{~Hz}, 2 \mathrm{H}, 2 \mathrm{x}$ aromatic H), 6.44 (t, $J=2.1 \mathrm{~Hz}, 1 \mathrm{H}$, aromatic $\mathrm{H}), 5.69$ (s, $1 \mathrm{H}, \mathrm{OH}), 3.95$ (s, 3H, $\mathrm{OCH}_{3}$ ), 3.85 (s, $\left.6 \mathrm{H}, 2 \times \mathrm{OCH}_{3}\right) .-{ }^{13} \mathrm{C} \mathrm{NMR}\left(\mathrm{CDCl}_{3}, 62.9 \mathrm{MHz}\right): \delta=161.1$ (s, 2 x aromatic C), 146.7 (s, aromatic C), 145.6 (s, aromatic C), 143.6 (s, aromatic C), 120.3 (d, aromatic $\mathrm{CH}$ ), 114.7 (d, aromatic $\mathrm{CH}$ ), 109.9 (d, aromatic $\mathrm{CH}), 105.4$ (d, $2 \mathrm{x}$ aromatic $\mathrm{CH}$ ), 98.7 (d, aromatic $\mathrm{CH}), 56.1$ (q, $\left.\mathrm{OCH}_{3}\right), 55.6$ (q, $2 \times \mathrm{OCH}_{3}$ ). - MS (EI) m/z (\%): 260 (100), 217 (60). - HRMS [M] ${ }^{+\bullet} \mathrm{C}_{15} \mathrm{H}_{16} \mathrm{O}_{4}$ : calcd. 260.10486; found: 260.1050 .

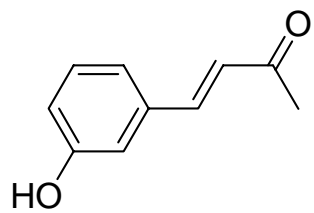

1) Dimethylmalonate, $\mathrm{Na}, \mathrm{MeOH}$ reflux, $4 \mathrm{~h}$

2) aq $\mathrm{NaOH}$, reflux, $1 \mathrm{~h}$

3) conc. $\mathrm{HCl}, 0^{\circ} \mathrm{C}$

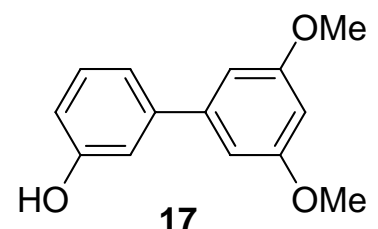

3',5'-Dimethoxy-biphenyl-3-ol (17). This product was obtained as a yellow oil (45\%) from 4-(3hydroxy-phenyl)-3-buten-2-one. - IR (film, NaCl): $v=3395 \mathrm{~cm}^{-1}, 2939,2838,1588,1418,1262$, 1204, 1154, 1064, 793, 692. ${ }^{1} \mathrm{H}$ NMR $\left(\mathrm{CDCl}_{3}, 300 \mathrm{MHz}\right): \delta=7.29(\mathrm{t}, J=7.9 \mathrm{~Hz}, 1 \mathrm{H}$, aromatic H), 7.15 (d, $J=6.3 \mathrm{~Hz}, 1 \mathrm{H}$, aromatic H), 7.05 (d, $J=0.9 \mathrm{~Hz}, 1 \mathrm{H}$, aromatic H), 6.84 (dd, $J=0.9$ and 6.6 $\mathrm{Hz}, 1 \mathrm{H}$, aromatic $\mathrm{H}), 6.72(\mathrm{~d}, J=6.6 \mathrm{~Hz}, 2 \mathrm{H}, 2 \mathrm{x}$ aromatic $\mathrm{H}), 6.49(\mathrm{~m}, 1 \mathrm{H}$, aromatic $\mathrm{H}), 5.60$ (broad s, 1H, OH), 3.84 (s, 6H, 2 x OCH $) .-{ }^{13} \mathrm{C} \mathrm{NMR}\left(\mathrm{CDCl}_{3}, 75.5 \mathrm{MHz}\right): \delta=161.0(\mathrm{~s}, 2 \mathrm{x}$ aromatic C), 156.0 (s, aromatic C), 143.2 (s, aromatic C), 143.0 (s, aromatic C), 130.1 (d, aromatic $\mathrm{CH}), 119.8$ (d, aromatic $\mathrm{CH}), 114.7$ (d, aromatic $\mathrm{CH}), 114.3(\mathrm{~d}$, aromatic $\mathrm{CH}), 105.6(\mathrm{~d}, 2 \mathrm{x}$ aromatic CH), 99.7 (d, aromatic CH), 55.6 (t, 2 x $\mathrm{OCH}_{3}$ ). - MS (EI) m/z (\%): 230 (20), 115 (100).

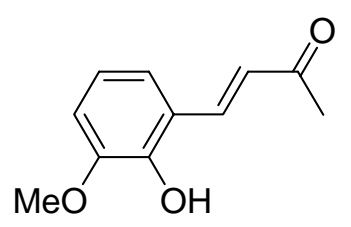

1) Dimethylmalonate, $\mathrm{Na}, \mathrm{MeOH}$ reflux, $4 \mathrm{~h}$

2) aq $\mathrm{NaOH}$, reflux, $1 \mathrm{~h}$

3) conc. $\mathrm{HCl}, 0^{\circ} \mathrm{C}$

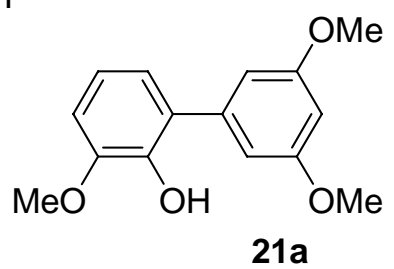

3,3',5'-Trimethoxy-biphenyl-2-ol (21a). This product was obtained as an oil (41\%) from 4-(2hydroxy-3-methoxy-phenyl)-3-buten-2-one. - IR (film, $\mathrm{NaCl}$ ): $v=3498 \mathrm{~cm}^{-1}, 2989,2840,1597$, 1461, 1417, 1281, 1233, 1204, 1154, 1063, 1033, 781, 761, 735. - ${ }^{1} \mathrm{H}$ NMR $\left(\mathrm{CDCl}_{3}, 250 \mathrm{MHz}\right): \delta=$ 7.02-6.86 (m, 3H, aromatic H), $6.79(\mathrm{~d}, J=2.1 \mathrm{~Hz}, 2 \mathrm{H}$, aromatic $\mathrm{H}), 6.49(\mathrm{t}, J=2.1 \mathrm{~Hz}, 1 \mathrm{H}$, aromatic $\mathrm{H}), 5.93(\mathrm{~s}, 1 \mathrm{H}, \mathrm{OH}), 3.94\left(\mathrm{~s}, 3 \mathrm{H}, \mathrm{OCH}_{3}\right), 3.84\left(\mathrm{~s}, 6 \mathrm{H}, 2 \times \mathrm{OCH}_{3}\right) .{ }^{13} \mathrm{C} \mathrm{NMR} \mathrm{CDCl}_{3}$, 
$62.9 \mathrm{MHz}$ ): $\delta=160.6$ (s, $2 \mathrm{x}$ aromatic C), 146.9 (s, aromatic C), 142.8 (s, aromatic C), 139.7 (s, aromatic C), 127.7 (s, aromatic C), 122.6 (d, aromatic CH), 119.8 (d, aromatic CH), 109.9 (d, aromatic $\mathrm{CH}$ ), 107.4 (d, 2 x aromatic $\mathrm{CH}$ ), 99.6 (d, aromatic $\mathrm{CH}), 56.3$ (q, $\left.\mathrm{OCH}_{3}\right), 55.4$ (q, 2 x $\left.\mathrm{OCH}_{3}\right)$. - HRMS [M] ${ }^{+\bullet} \mathrm{C}_{15} \mathrm{H}_{16} \mathrm{O}_{4}$ : calcd. 260.10486; found: 260.1050.

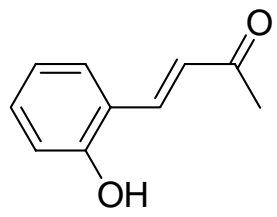

1) Dimethylmalonate, $\mathrm{Na}, \mathrm{MeOH}$ reflux, $4 \mathrm{~h}$

2) aq $\mathrm{NaOH}$, reflux, $1 \mathrm{~h}$

3) conc. $\mathrm{HCl}, 0^{\circ} \mathrm{C}$

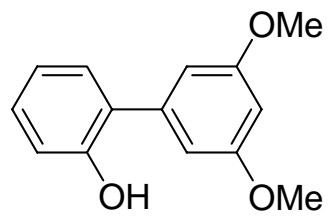

21b

3',5'-Dimethoxy-biphenyl-2-ol (21b). This product was obtained as a yellow oil (38\%) from 4-(2hydroxy-phenyl)-3-buten-2-one. - IR (film, NaCl): $v=3444 \mathrm{~cm}^{-1}, 2939,2838,1594,1418,1204$, 1154, 1063, 756. - ${ }^{1} \mathrm{H} \mathrm{NMR}\left(\mathrm{CDCl}_{3}, 250 \mathrm{MHz}\right): \delta=7.69-7.63$ (m, 2H, aromatic H), 7.42-7.36 (m, $2 \mathrm{H}$, aromatic $\mathrm{H}$ ), $7.02(\mathrm{~d}, J=2.1 \mathrm{~Hz}, 1 \mathrm{H}$, aromatic $\mathrm{H}$ ), $6.89(\mathrm{t}, J=2.1 \mathrm{~Hz}$, aromatic $\mathrm{H}$ ), 6.12 (broad s, $1 \mathrm{H}, \mathrm{OH}), 4.20$ (s, $\left.6 \mathrm{H}, 2 \times \mathrm{OCH}_{3}\right) .{ }^{13} \mathrm{C} \mathrm{NMR}\left(\mathrm{CDCl}_{3}, 62.9 \mathrm{MHz}\right): \delta=161.4$ (s, 2 x aromatic C), 152.5 (s, C-OH), 139.1 (s, aromatic C), 130.0 (d, aromatic CH), 129.3 (d, aromatic CH), 128.0 (s, aromatic C), 120.7 (d, aromatic $\mathrm{CH}), 115.9$ (d, aromatic $\mathrm{CH}), 107.0$ (d, 2 x aromatic $\mathrm{CH}), 99.9$ (d, aromatic CH), 55.4 (q, $2 \times \mathrm{OCH}_{3}$ ). - MS (ESI) m/z (\%): $231[\mathrm{M}+\mathrm{H}]^{+}$(100) $216\left[\mathrm{M}+\mathrm{H}-\mathrm{CH}_{3}\right]^{+}$(18). HRMS [M+H] ${ }^{+} \mathrm{C}_{14} \mathrm{H}_{15} \mathrm{O}_{3}$ : calcd. 231.1021; found: 231.1021.

\section{Other Biaryls:}
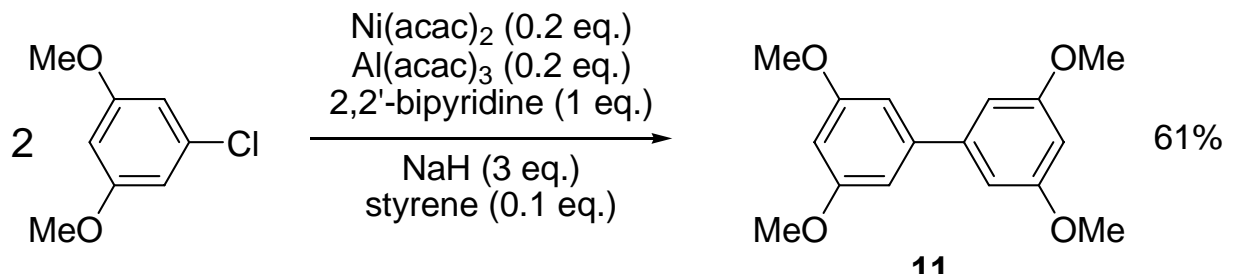

3,5,3',5'-Tetramethoxy-biphenyl (11). ${ }^{7} \quad$ In a dry three necked flask equipped with a reflux condenser and a dropping funnel, nickel(II) acetylacetonate (0.642 g, $2.5 \mathrm{mmol})$, aluminium(III) acetylacetonate $(0.811 \mathrm{~g}, 2.5 \mathrm{mmol})$ and 2,2'-bipyridine (1.952 g, $12.5 \mathrm{mmol})$ were added to a suspension of sodium hydride $(0.900 \mathrm{~g}, 37.5 \mathrm{mmol})$ in THF $(30 \mathrm{~mL})$ and the mixture was stirred further at $65{ }^{\circ} \mathrm{C}$ for 8 hours. 5-Chloro-1,3-dimethoxybenzene (4.315 g, $25 \mathrm{mmol}$ ) and styrene (143 $\mu \mathrm{L}, 1.25 \mathrm{mmol})$ in THF (10 mL) were then added to the black suspension and the mixture was stirred further at reflux for 4 hours. After cooling to room temperature, water (1 mL) and DCM (50 $\mathrm{mL}$ ) were added and the reaction mixture was filtered. After drying over sodium sulfate and evaporation of the solvents, the crude product was purified by silica gel chromatography (Petroleum ether/EtOAc, 9:1) to give 3,5,3',5'-tetramethoxy-biphenyl as a white solid (2.106 g, 7.69 mmol, 61 $\%$ ), which could be recrystallized in DCM/hexane. - m.p. 106.2-107.1 ${ }^{\circ} \mathrm{C}$. - IR (solid, $\mathrm{KBr}$ ): $v=$ $2937 \mathrm{~cm}^{-1}, 1606,1579,1471,1410,1293,1197,1162,1031,847 .-{ }^{1} \mathrm{H}$ NMR $\left(\mathrm{CDCl}_{3}, 250 \mathrm{MHz}\right): \delta$ $=6.72(\mathrm{~d}, J=2.2 \mathrm{~Hz}, 4 \mathrm{H}, 4 \mathrm{x}$ aromatic $\mathrm{CH}), 6.48(\mathrm{t}, J=2.2 \mathrm{~Hz}, 2 \mathrm{H}, 2 \mathrm{x}$ aromatic $\mathrm{CH}), 3.85(\mathrm{~s}$, $\left.12 \mathrm{H}, 4 \times \mathrm{OCH}_{3}\right)$. - ${ }^{13} \mathrm{C} \mathrm{NMR}\left(\mathrm{CDCl}_{3}, 62.9 \mathrm{MHz}\right): \delta(\mathrm{ppm})=161.1$ (s, $4 \mathrm{x}$ aromatic $\left.\mathrm{CH}\right), 143.6(\mathrm{~s}, 2$ $\mathrm{x}$ aromatic $\mathrm{CH}), 105.7$ (d, $4 \mathrm{x}$ aromatic $\mathrm{CH}), 99.6$ (d, $2 \mathrm{x}$ aromatic $\mathrm{CH}), 55.6$ (q, $4 \mathrm{x} \mathrm{OCH}_{3}$ ). HRMS [M+H] $]^{+} \mathrm{C}_{16} \mathrm{H}_{19} \mathrm{O}_{4}$ : calcd. 275.12833; found: 275.12867. - Anal. Calcd for $\mathrm{C}_{16} \mathrm{H}_{18} \mathrm{O}_{4}$ (274.12): C 70.06, H 6.61; found: C 69.99, H 6.65.

\footnotetext{
${ }^{7}$ Following a previously published procedure: Massicot, F.; Schneider, R.; Fort, Y.; Illy-Cherrey, S.; Tillement, O.
} Tetrahedron 2001, 57, 531-536. 


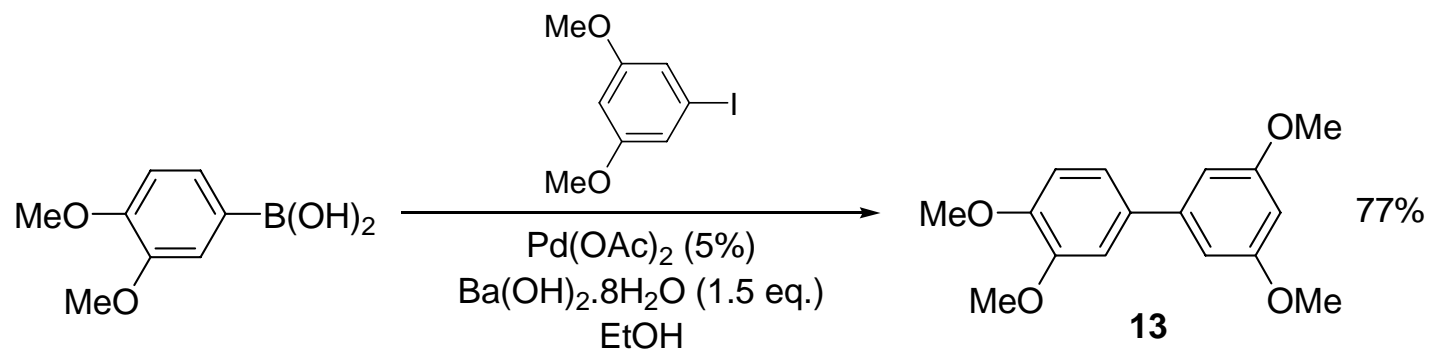

3,4,3',5'-Tetramethoxy-biphenyl (13). ${ }^{8}$ In a $100 \mathrm{~mL}$ two-necked flask surrounded with a reflux condenser was added 3,4-dimethoxybenzene boronic acid (0.728 g, $4 \mathrm{mmol}, 1$ eq.), iodo-3,5dimethoxybenzene (1.162 g, $4.4 \mathrm{mmol}, 1.1 \mathrm{eq}$.), barium hydroxide octahydrate (1.893 g, $6 \mathrm{mmol}$, 1.5 eq.) in ethanol $(40 \mathrm{~mL})$. The flask was submitted to 3 freeze-pump-thaw cycles and palladium acetate $(0.045 \mathrm{~g}, 0.2 \mathrm{mmol}, 0.05$ eq.) was added under nitrogen. After five minutes, the mixture turned black. After stirring 16 hours at room temperature, the solution was filtrated on celite, washed with ethyl acetate, dried over $\mathrm{Na}_{2} \mathrm{SO}_{4}$. Evaporation of the solvents gave a brown paste, which was purified by silica gel chromatography (Petroleum ether/EtOAc, 4:1). 3,4,3',5'Tetramethoxybiphenyl was obtained as a white solid (0.839 g, $3.06 \mathrm{mmol}, 77 \%$ ) which could be recrystallized with DCM/hexane. - m.p. 99.7-100.5 ${ }^{\circ} \mathrm{C}$. - IR (solid, KBr): $v=3000 \mathrm{~cm}^{-1}$, 2833, 1598, 1579, 1517, 1261, 1209, 1158, 1072, 1028, 829, 808, 762. - ${ }^{1} \mathrm{H}$ NMR $\left(\mathrm{CDCl}_{3}, 300 \mathrm{MHz}\right): \delta=$ 7.16-7.10 (m, 2H, aromatic H), $6.93(\mathrm{~d}, J=8.3 \mathrm{~Hz}, 1 \mathrm{H}$, aromatic H), 6.70 (d, $J=2.3 \mathrm{~Hz}$, aromatic $\mathrm{H}), 6.45$ (t, $J=2.3 \mathrm{~Hz}, 1 \mathrm{H}$, aromatic $\mathrm{CH}), 3.94$ (s, 3H, $\left.\mathrm{OCH}_{3}\right), 3.91$ (s, 3H, $\left.\mathrm{OCH}_{3}\right), 3.84(\mathrm{~s}, 6 \mathrm{H}, 2 \mathrm{x}$ $\left.\mathrm{OCH}_{3}\right) .-{ }^{13} \mathrm{C} \mathrm{NMR}\left(\mathrm{CDCl}_{3}, 75.5 \mathrm{MHz}\right): \delta=161.1$ (s, 2 x aromatic C), 149.1 (s, aromatic C), 148.8 (s, aromatic C), 143.3 (s, aromatic C), 134.2 (s, aromatic C), 119.4 (d, aromatic CH), 111.3 (d, aromatic $\mathrm{CH}$ ), 110.5 (d, aromatic $\mathrm{CH}), 105.3$ (d, 2 x aromatic $\mathrm{CH}), 98.6$ (d, aromatic $\mathrm{CH}), 56.0$ (q, $2 \mathrm{x} \mathrm{OCH}_{3}$ ), 55.4 (q, $\left.2 \times \mathrm{OCH}_{3}\right)$. - MS (SIMS) m/z (\%): $274[\mathrm{M}]^{+\bullet}(100)$. - HRMS [M] ${ }^{+\bullet} \mathrm{C}_{16} \mathrm{H}_{18} \mathrm{O}_{4}$ : calcd. 274.1205; found: 274.1213. - Anal. Calcd for $\mathrm{C}_{16} \mathrm{H}_{18} \mathrm{O}_{4}$ (274.12): C 70.06, H 6.61; found: C 70.31, H 6.62 .

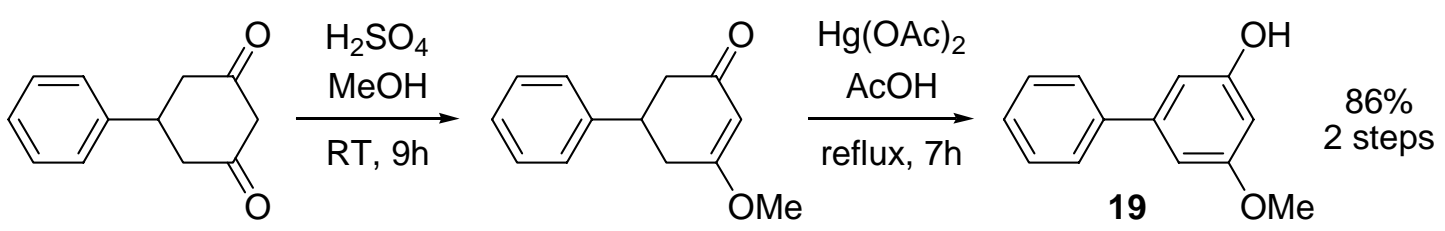

5-Methoxy-biphenyl-3-ol (19). ${ }^{9}$ Phenylcyclohexadienone (4.70 g, $25 \mathrm{mmol}$ ) was dissolved in methanol $(200 \mathrm{~mL})$. Sulfuric acid $(0.50 \mathrm{~g})$ was added and the solution was allowed to stir at room temperature until thin layer chromatography (pentane/THF, 1:1) showed the disappearance of the starting material (9 hours). Methanol was evaporated. DCM was then added and the organic layer was washed with a saturated $\mathrm{NaHCO}_{3}$ solution, brine, and dried with sodium sulfate. Evaporation of the solvent gave $4.68 \mathrm{~g}$ of a yellow oil which could be used without purification in the next step.

The resulting 3-methoxy-5-phenyl-cyclohexenone (3.12 g, $15.4 \mathrm{mmol})$ was diluted in glacial acetic acid $(43 \mathrm{~mL})$ and mercury acetate $(9.01 \mathrm{~g}, 28.3 \mathrm{mmol})$ was added. The solution was refluxed for $7 \mathrm{~h}$, cooled and filtrated. The salts were washed with ether and the filtrate was combined with the acetic acid phase. An aqueous sodium hydroxide solution was added until neutral $\mathrm{pH}$. The aqueous phase was extracted with ether. The organic layer was washed with brine and dried over sodium sulfate. Evaporation of the solvent gave a brown paste which was purified by chromatography through flash silica gel (Petroleum ether/EtOAc, 9:1) to give 5-methoxy-biphenyl-3-ol as a yellow oil (2.87 g, 14 mmol, $86 \%$ calculated from phenylcyclohexadienone). - IR (film, NaCl): $v=3404 \mathrm{~cm}^{-1}, 3060$, 2940, 2841, 1596, 1478, 1422, 1349, 1152, 1050, 970, 842, 762, 697. - ${ }^{1} \mathrm{H}$ NMR $\left(\mathrm{CDCl}_{3}, 250\right.$

\footnotetext{
${ }^{8}$ Following a previously published procedure : Campi, E. M.; Jackson, W. R.; Marcuccio, S. M.; Naeslund, C. M. J. Chem. Soc. Chem. Comm. 1994, 2395.

9 Itoh, Y.; Brossi, A.; Hamel, E.; Lin, C. M. Helv. Chim. Acta 1988, 71, 1199-1209.
} 
MHz): $\delta=7.58-7.54(\mathrm{~m}, 2 \mathrm{H}$, aromatic $\mathrm{H}), 7.46-7.32(\mathrm{~m}, 3 \mathrm{H}$, aromatic $\mathrm{H}), 6.74-6.73(\mathrm{~m}, 1 \mathrm{H}$, aromatic H), 6.68-6.66 m, 1H, aromatic $\mathrm{H}$ ), $6.43(\mathrm{t}, J=2.1 \mathrm{~Hz}, 1 \mathrm{H}$, aromatic $\mathrm{H}), 4.42$ (broad s, $1 \mathrm{H}$, $\mathrm{OH}), 3.84\left(\mathrm{~s}, 3 \mathrm{H}, \mathrm{OCH}_{3}\right) .{ }^{13} \mathrm{C} \mathrm{NMR}\left(\mathrm{CDCl}_{3}, 62.9 \mathrm{MHz}\right): \delta=161.1$ (s, aromatic C), 152.9 (s, aromatic C), 143.8 (s, aromatic C), 140.7 (aromatic C), 128.8, (d, 2 x aromatic CH), 127.7 (d, aromatic $\mathrm{CH}$ ), 127.1 (d, 2 x aromatic $\mathrm{CH}$ ), 107.2 (d, aromatic $\mathrm{CH}), 105.9$ (d, aromatic $\mathrm{CH}), 100.6$ (d, aromatic CH), 55.5 (q, $\mathrm{OCH}_{3}$ ). - MS (EI) m/z (\%): 200 [M] ${ }^{+\bullet}$ (100), 171 (35), 128 (33).

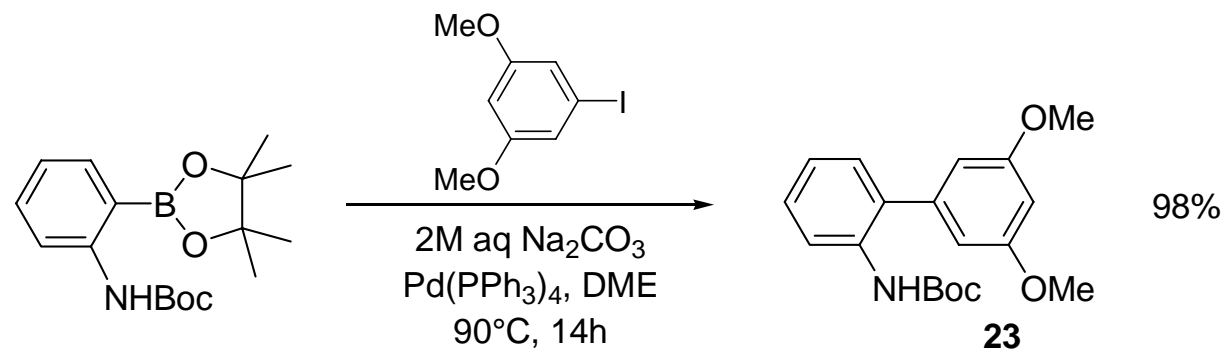

3',5'-Dimethoxybiphenyl-2-carbamic acid tert-butyl ester (23). ${ }^{10}$ [2-(4,4,5,5-Tetramethyl[1,3,2]dioxaborolan-2-yl)-phenyl]-carbamic acid tert-butyl ester (0.638 g, $2 \mathrm{mmol})$ and iodo-3,5dimethoxybenzene $(0.634 \mathrm{~g}, 2.2 \mathrm{mmol})$ were added in a $2 \mathrm{M}$ aqueous solution of $\mathrm{Na}_{2} \mathrm{CO}_{3}(2 \mathrm{~mL})$ and DME $(5 \mathrm{~mL})$ in a $25 \mathrm{~mL}$ two-necked flask equipped with a condenser. Dioxygene was extruded by three freeze-pump-thaw cycles. Palladium(tetrakistriphenylphosphine) (0.063g, 0.06 mmol) was then added under nitrogen. The mixture was warmed to $90{ }^{\circ} \mathrm{C}$ for 14 hours. Filtration over celite, washing with ethyl acetate, drying with $\mathrm{Na}_{2} \mathrm{SO}_{4}$ and evaporation of solvents gave a brown paste which was purified by silica gel chromatography (Petroleum ether/EtOAc, 95:5). 3',5'-Dimethoxy-biphenyl-2-carbamic acid tert-butyl ester (0.650 g, $1.98 \mathrm{mmol}, 98 \%)$ was obtained as a white solid, which could be recrystallised in DCM/hexane. - m.p. 88.0-88.8 ${ }^{\circ} \mathrm{C}$. - IR (solid, $\mathrm{KBr}): v=3422 \mathrm{~cm}^{-1}$,3351, 2976, 1728, 1697, 1589, 1518, 1456, 1247, 1205, 1159, 1061, 848, 754. - ${ }^{1} \mathrm{H}$ NMR $\left(\mathrm{CDCl}_{3}, 250 \mathrm{MHz}\right): \delta=8.13(\mathrm{~d}, J=8.2 \mathrm{~Hz}, 1 \mathrm{H}$, aromatic H), 7.34 (td, $J=1.5$ and 7.8 $\mathrm{Hz}, 1 \mathrm{H}$, aromatic $\mathrm{H}), 7.24-7.20$ (m, $1 \mathrm{H}$, aromatic $\mathrm{H}), 7.12-7.05(\mathrm{~m}, 1 \mathrm{H}$, aromatic $\mathrm{H}), 6.65$ (broad s, $1 \mathrm{H}, \mathrm{NH}), 6.51(\mathrm{~s}, 3 \mathrm{H}$, aromatic $\mathrm{H}), 3.82\left(\mathrm{~s}, 6 \mathrm{H}, 2 \times \mathrm{OCH}_{3}\right), 1.49(\mathrm{~s}, 9 \mathrm{H}, t-\mathrm{Bu}) .-{ }^{13} \mathrm{C} \mathrm{NMR}\left(\mathrm{CDCl}_{3}\right.$, $62.9 \mathrm{MHz}$ ): $\delta=161.0$ (s, 2 x Cq-OMe), 152.6 (s, C=O), 140.1 (s, Cq-NH), 135.1 (s, aromatic C), 131.2 (s, aromatic C), 129.6 (d, aromatic $\mathrm{CH}), 128.1$ (d, aromatic $\mathrm{CH}), 122.8$ (d, aromatic $\mathrm{CH})$, 119.7 (d, aromatic CH), 106.9 (d, 2 x aromatic $\mathrm{CH}$ ), 99.7 (d, aromatic $\mathrm{CH}$ ), 80.0 (s, aliphatic C), 55.0 (q, $2 \times \mathrm{OCH}_{3}$ ), 28.0 (q, $3 \times \mathrm{CH}_{3}$ ). - Anal. Calcd for $\mathrm{C}_{19} \mathrm{H}_{23} \mathrm{NO}_{4}$ (329.39): C 69.28, H 7.04, N 4.25; found: C 69.18, H 7.28, N 4.18. - MS (EI) m/z (\%): $329[\mathrm{M}]^{+\bullet}$ (11), $273[\mathrm{M}+\mathrm{H}-\mathrm{tBu}]^{+\bullet}(42)$, $229\left[\mathrm{M}+\mathrm{H}-\mathrm{tBu}-\mathrm{CO}_{2}\right]^{+\bullet}(100)$.

\section{D) General procedure for Birch reductive alkylation:}

In a dry three-necked flask equipped with a dry-ice condenser was introduced, under nitrogen, a biaryl $(8 \mathrm{mmol})$ in THF $(40 \mathrm{~mL})$. In case the starting material is a phenol, $n$-BuLi $(2.5 \mathrm{M}$ sol. in hexane, 1.1 eq.) was added dropwise at $-78{ }^{\circ} \mathrm{C}$ and the solution was stirred for 10 minutes.

The flask was then cooled to $-78{ }^{\circ} \mathrm{C}$ and ammonia (approx. $80 \mathrm{~mL}$ ) was condensed. Lithium wire ( $0.133 \mathrm{~g}, 19 \mathrm{mmol}, 2.3$ eq.) was added. The solution was stirred either at $-78{ }^{\circ} \mathrm{C}$ for 30 minutes in case of "activated" biaryls (e.g. containing a 3,5-dimethoxyphenyl ring) or stirred at $-33{ }^{\circ} \mathrm{C}$ (refluxing $\mathrm{NH}_{3}$ ) for $1 \mathrm{~h}$ for the others. The red mixture was then cooled to $-78{ }^{\circ} \mathrm{C}$ and chloroacetonitrile (1.6 mL, $24 \mathrm{mmol}, 3$ eq.) in THF $(10 \mathrm{~mL})$ was added in one portion (Methyl chloroacetate was added for 10a). The mixture turned immediately brown. After 5 minutes,

\footnotetext{
${ }^{10}$ Following a previously published procedure : Lamba, J. J. S.; Tour, J. M. J. Am. Chem. Soc. 1994, 116, $11723-11736$.
} 
ammonia was let to evaporate and a half-saturated aqueous ammonium chloride solution was added. Extraction with ether, washing with brine, drying with sodium sulfate and evaporation of the solvents gave a brown paste, which was purified by silica gel chromatography (Petroleum ether/EtOAc mixtures)

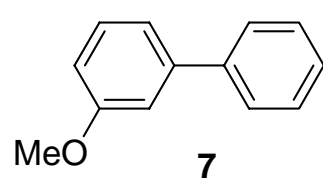

7

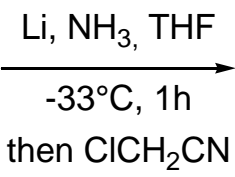

then $\mathrm{ClCH}_{2} \mathrm{CN}$

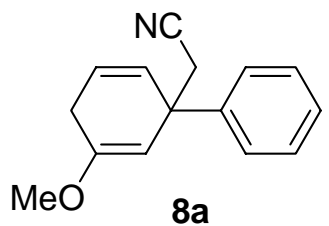

$8 \mathbf{a}$

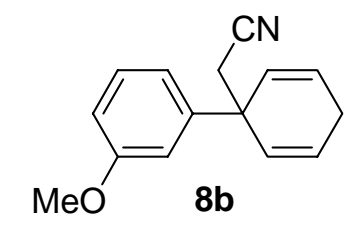

8a : 8 b

$77: 23(72 \%)$

The 2 compounds have too close $\mathrm{Rf}$ to be separated by silica gel flash chromatography. See below the copy of ${ }^{1} \mathrm{H}$ and ${ }^{13} \mathrm{C}$ spectra of the purified mixture. (the 77:23 ratio was determined on the crude reaction mixture).

Mixture: IR (solid, KBr): $v=3443 \mathrm{~cm}^{-1}$, 3029, 2957, 2247 (CN), 1684, 1644, 1599, 1446, 1380, 1220, 1172, 698. - MS (SIMS) m/z (\%): $248[\mathrm{M}+\mathrm{Na}]^{+}$(63), $\left.226[\mathrm{M}+\mathrm{H}]^{+}(100) .-\mathrm{HRMS}^{\mathrm{M}}+\mathrm{Na}\right]^{+}$ $\mathrm{C}_{15} \mathrm{H}_{15} \mathrm{NNaO}$ : Calcd 248.1051; found: 248.1049.

(3-Methoxy-1-phenyl-cyclohexa-2,5-dienyl)-acetonitrile (8a). ${ }^{1} \mathrm{H}$ NMR $\left(\mathrm{CDCl}_{3}, 250 \mathrm{MHz}\right): \delta=$ 7.40-7.20 (m, 4H, aromatic H), 5.98-5.85 (td, $J=3.4,10.1 \mathrm{~Hz}, 1 \mathrm{H}$, vinylic $\mathrm{H}), 5.75-5.68(\mathrm{~m}, 1 \mathrm{H}$, vinylic $\mathrm{H}$ ), 4.72 (s, $1 \mathrm{H}$, vinylic $\mathrm{H}$ ), 3.61 (s, $\left.3 \mathrm{H}, \mathrm{OCH}_{3}\right), 3.0-2.70(\mathrm{~m}, 2 \mathrm{H}$, allylic $\mathrm{CH} a \mathrm{H} b), 2.90$ (s, $\left.2 \mathrm{H}, \mathrm{CH}_{2} \mathrm{CN}\right)$.

[1-(3-Methoxy-phenyl)-cyclohexa-2,5-dienyl]-acetonitrile (8b). ${ }^{1} \mathrm{H} \mathrm{NMR}\left(\mathrm{CDCl}_{3}, 250 \mathrm{MHz}\right): \delta$ $=7.30-7.20(\mathrm{~m}, 1 \mathrm{H}$, aromatic $\mathrm{H}), 6.95-6.75(\mathrm{~m}, 3 \mathrm{H}$, aromatic $\mathrm{H}), 6.05-5.98(\mathrm{~m}, 2 \mathrm{H}$, vinylic $\mathrm{H})$, 5.76-5.67 (m, 2H, vinylic $\mathrm{H}), 3.80\left(\mathrm{~s}, 3 \mathrm{H}, \mathrm{OCH}_{3}\right), 2.88-2.65(\mathrm{~m}, 2 \mathrm{H}, \mathrm{CH} a \mathrm{Hb}), 2.82(\mathrm{~s}, 2 \mathrm{H}$, $\left.\mathrm{CH}_{2} \mathrm{CN}\right)$.
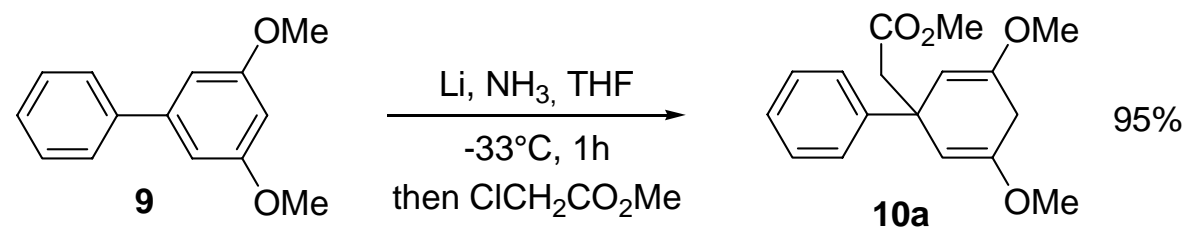

(3,5-Dimethoxy-1-phenyl-cyclohexa-2,5-dienyl)-acetic acid methyl ester (10a). This product was obtained as a yellow oil (95\%). - IR (film, $\mathrm{NaCl}$ ): $v=3000 \mathrm{~cm}^{-1}, 2951,2904,2828,1738$, 1694, 1444, 1400, 1236, 1202, 1151, 1021, 764, 700. - ${ }^{1} \mathrm{H}$ NMR $\left(\mathrm{CDCl}_{3}, 300 \mathrm{MHz}\right): \delta=7.40-7.29$ (m, 4H, aromatic $\mathrm{CH}), 7.17-7.12(\mathrm{~m}, 1 \mathrm{H}$, aromatic $\mathrm{CH}), 4.86(\mathrm{~s}, 2 \mathrm{H}, 2 \mathrm{x}$ vinylic $\mathrm{CH}), 3.60$ (s, $6 \mathrm{H}$, $\left.2 \mathrm{x} \mathrm{OCH}_{3}\right), 3.58\left(\mathrm{~s}, 3 \mathrm{H}, \mathrm{CO}_{2} \mathrm{H}_{3}\right), 2.95\left(\mathrm{~s}, 2 \mathrm{H}, \mathrm{CH}_{2} \mathrm{CO}_{2} \mathrm{Me}\right), 2.83(\mathrm{~s}, 2 \mathrm{H}, \mathrm{CHaH} b) .-{ }^{13} \mathrm{C} \mathrm{NMR}$ $\left(\mathrm{CDCl}_{3}, 75.5 \mathrm{MHz}\right): \delta=171.5$ (s, C=O), 151.6 (s, 2 x aromatic C), 148.3 (s, aromatic C), 128.4 (d, $2 \mathrm{x}$ aromatic $\mathrm{CH}$ ), 126.1 (d, aromatic $\mathrm{CH}$ ), 126.0 (2 x aromatic $\mathrm{CH}), 100.2$ (d, 2 x vinylic $\mathrm{CH}), 54.4$ (q, 2 x $\mathrm{OCH}_{3}$ ), 51.3 (q, $\mathrm{CO}_{2} \underline{\mathrm{CH}} 3$ ), 47.2 (t, $\mathrm{CH}_{2} \mathrm{CO}_{2} \mathrm{Me}$ ), 45.0 (s, aliphatic C), 31.0 (t, $\mathrm{CHaHb}$ ). - MS

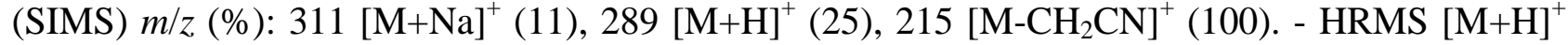
$\mathrm{C}_{17} \mathrm{H}_{21} \mathrm{O}_{4}$ : calcd 289.1440; found: 289.1441. 

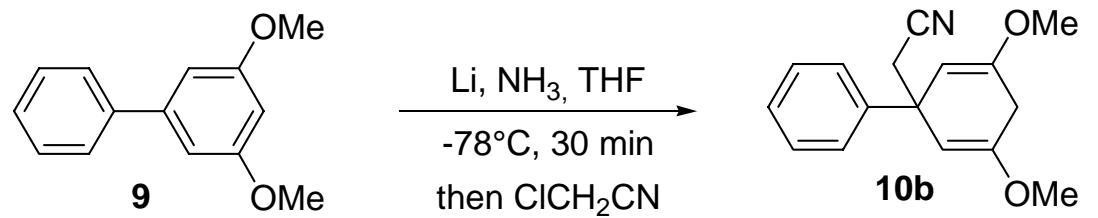

$88 \%$

(3,5-Dimethoxy-1-phenyl-cyclohexa-2,5-dienyl)-acetonitrile (10b). This product was obtained as a white solid (88\%) which could be recrystallized in DCM/hexane. - m.p. 102.5-103.5 ${ }^{\circ} \mathrm{C}$. - IR (solid, KBr): $v=2929 \mathrm{~cm}^{-1}$, 2252, 1695, 1406, 1210, 1196, 1165, 1148, 1024, 870, 767, 704. - ${ }^{1} \mathrm{H}$ NMR $\left(\mathrm{CDCl}_{3}, 250 \mathrm{MHz}\right): \delta=7.36-7.33(\mathrm{~m}, 4 \mathrm{H}$, aromatic $\mathrm{H}), 7.29-7.22$ (m, 1H, aromatic $\left.\mathrm{H}\right), 4.70$ (s, 2H, 2 x vinylic H), 3.60 (s, 6H, 2 x $\mathrm{OCH}_{3}$ ), 2.93 (s, 2H, $\mathrm{CH}_{2} \mathrm{CN}$ ) 2.91 (AB system, $J_{a b}=17.7$ $\mathrm{Hz}, 2 \mathrm{H}, \mathrm{CH} a \mathrm{Hb}$ ). $-{ }^{13} \mathrm{C} \mathrm{NMR}\left(\mathrm{CDCl}_{3}, 62.9 \mathrm{MHz}\right): \delta=153.2$ (s, $2 \mathrm{x}$ aromatic C), 146.3 (s, aromatic C), 128.6 (d, aromatic CH), 126.7 (d, aromatic CH), 125.8 (d; aromatic CH), 118.1 (s, CN), 98.4 (d, 2 x vinylic $\mathrm{CH}$ ), 54.4 (q, $\left.2 \times \mathrm{OCH}_{3}\right), 44.9$ (s, aliphatic C), 32.1 (t, $\mathrm{CH}_{2} \mathrm{CN}$ ), 31.1 (t, $\mathrm{CH} a \mathrm{Hb}$ ). - MS

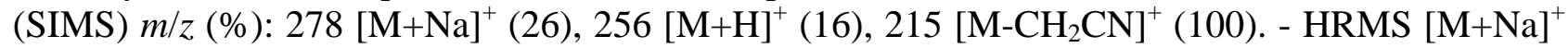
$\mathrm{C}_{16} \mathrm{H}_{17} \mathrm{NNaO}_{2}$ : Calcd 278.1157; found: 278.1160. - Anal. Calcd for $\mathrm{C}_{16} \mathrm{H}_{17} \mathrm{NO}_{2}$ (255.31): C 75.25, H 6.71, N 5.49; found: C 74.70, H 6.72, N, 5.41.<smiles>COc1cc(OC)cc(-c2cc(OC)cc(OC)c2)c1</smiles>

11

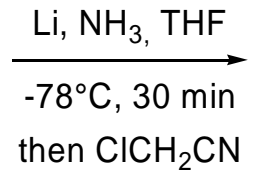

then $\mathrm{ClCH}_{2} \mathrm{CN}$<smiles>COC1=CC(CC#N)(c2cc(OC)cc(OC)c2)C=C(OC)C1</smiles>

12

$91 \%$

[1-(3,5-Dimethoxy-phenyl)-3,5-dimethoxy-cyclohexa-2,5-dienyl]-acetonitrile (12). This product was obtained as a white solid (91\%) and could be recrystallized in hexane/DCM. - m.p. 120.6-121.8 ${ }^{\circ} \mathrm{C}$. - IR (solid, $\mathrm{KBr}$ ): $v=2967 \mathrm{~cm}^{-1}, 2251(\mathrm{CN}), 1690,1604,1586,1311,1207,1160,1067,832$, 208, 196. $-{ }^{1} \mathrm{H} \mathrm{NMR}\left(\mathrm{CDCl}_{3}, 250 \mathrm{MHz}\right): \delta=6.48(\mathrm{~d}, J=2.1 \mathrm{~Hz}, 2 \mathrm{H}, 2 \mathrm{x}$ aromatic $\mathrm{CH}), 6.34(\mathrm{t}, J=$ $2.1 \mathrm{~Hz}, 1 \mathrm{H}$, aromatic $\mathrm{H}$ ), 4.67 (s, $2 \mathrm{H}, 2 \mathrm{x}$ vinylic $\mathrm{H}$ ), 3.79 (s, $6 \mathrm{H}, 2 \mathrm{x}$ aromatic $\left.\mathrm{OCH}_{3}\right), 3.59$ (s, 6H, 2 x $\left.\mathrm{OCH}_{3}\right), 2.89\left(\mathrm{AB}\right.$ system, $\left.J_{a b}=18.9 \mathrm{~Hz}, 2 \mathrm{H}, \mathrm{CHaHb}\right), 2.87\left(\mathrm{~s}, 2 \mathrm{H}, \mathrm{CH}_{2} \mathrm{CN}\right) .-{ }^{13} \mathrm{C} \mathrm{NMR}\left(\mathrm{CDCl}_{3}\right.$, $62.9 \mathrm{MHz}$ ): $\delta=161.0$ (s, 2 x aromatic C), 153.5 (s, 2 x vinylic C), 149.0 (s, aromatic C), 118.1 (s, $\mathrm{CN}$ ), 104.7 (d, $2 \mathrm{x}$ aromatic $\mathrm{CH}$ ), 98.2 (d, $2 \mathrm{x}$ vinylic $\mathrm{CH}) 97.7$ (d, aromatic $\mathrm{CH}$ ), 55.4 (d, $2 \mathrm{x}$ OCH3), 54.6 (d, $2 \times \mathrm{OCH}_{3}$ ), 45.1 (s, aliphatic C), 32.3 (t, $\mathrm{CH}_{2} \mathrm{CN}$ ), 31.1 (t, $\mathrm{CH} a \mathrm{Hb}$ ). - MS (SIMS) $\mathrm{m} / \mathrm{z}(\%): 338[\mathrm{M}+\mathrm{Na}]^{+}$(31), $316[\mathrm{M}+\mathrm{H}]^{+}(24), 275\left[\mathrm{M}-\mathrm{CH}_{2} \mathrm{CN}\right]^{+}$(100). - HRMS [M+Na] $\mathrm{C}_{18} \mathrm{H}_{21} \mathrm{NO}_{4} \mathrm{Na}$ : calcd 338.1368; found: 338.1370. - Anal. Calcd for $\mathrm{C}_{18} \mathrm{H}_{21} \mathrm{NO}_{4}$ (315.36): C 68.55, $\mathrm{H}$ 6.71, N 4.44; found: C 68.22, H 6.70, N 4.37.
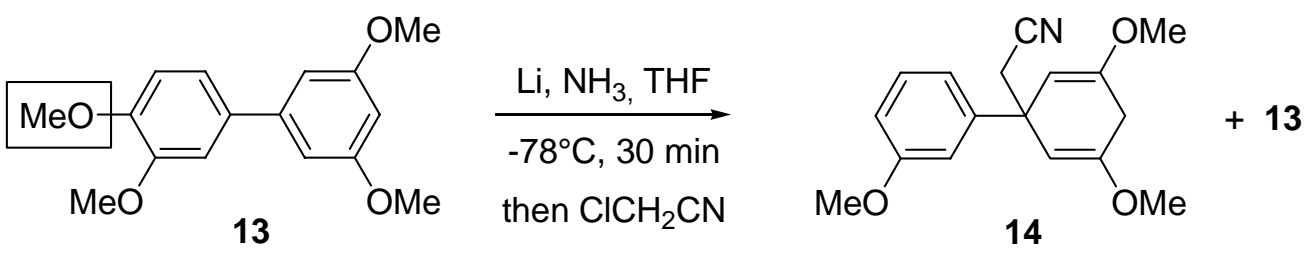

$14: 13$

$1: 1$

This reaction has no preparative value. Compound $\mathbf{1 4}$ is better prepared in 2 steps by methylation $\left(\mathrm{Me}_{2} \mathrm{SO}_{4}, \mathrm{~K}_{2} \mathrm{CO}_{3}\right.$, acetone, 90\% yield) of compound 18 (the following description of compound 14 comes from this reaction). 
[3,5-Dimethoxy-1-(3-methoxy-phenyl)-cyclohexa-2,5-dienyl]-acetonitrile (14). White solid. m.p. 102.8-103.5 ${ }^{\circ} \mathrm{C}$. - IR (solid, KBr): $v=2966 \mathrm{~cm}^{-1}$, 2247 (CN), 1695, 1605, 1404, 1244, 1210, 1156, 1044, 838, 786, 701. - ${ }^{1} \mathrm{H}$ NMR $\left(\mathrm{CDCl}_{3}, 300 \mathrm{MHz}\right): \delta=7.27$ (t, $J=7.9 \mathrm{~Hz}, 1 \mathrm{H}$, aromatic H), 6.95-6.88 (m, 2H, aromatic $\mathrm{H}), 6.80-6.77(\mathrm{~m}, 1 \mathrm{H}$, aromatic $\mathrm{H}), 4.70$ (s, $2 \mathrm{H}, 2$ x vinylic $\mathrm{H}), 3.81$ (s, $3 \mathrm{H}, \mathrm{OCH}_{3}$ ), 3.60 (s, 6H, 2 x $\mathrm{OCH}_{3}$ ), 2.91 (AB system, $J_{a b}=22.9 \mathrm{~Hz}, 2 \mathrm{H}, \mathrm{CHaHb}$ ), 2.90 (s, 2H, $\left.\mathrm{CH}_{2} \mathrm{CN}\right) .-{ }^{13} \mathrm{C} \mathrm{NMR}\left(\mathrm{CDCl}_{3}, 75.5 \mathrm{MHz}\right): \delta=159.8$ (s, aromatic C), 153.5 (s, 2 x vinylic C), 148.2 (s, aromatic C), 129.7 (d, aromatic CH), 118.1 (s, CN), 118.0 (d, aromatic $\mathrm{CH}$ ), 98.4 (d, 2 x vinylic $\mathrm{CH}$ ), 55.3 (q, $\mathrm{OCH}_{3}$ ), 54.6 (q, 2 x $\mathrm{OCH}_{3}$ ), 45.0 (s, aliphatic C), 32.3 (t, $\mathrm{CH}_{2} \mathrm{CN}$ ), 31.2 (t, $\mathrm{CHaHb}$ ). HRMS [M-CH (315.36): C, 71.56; H, 6.71; N, 4.91; found: C 71.74, H 6.78, N 4.82.
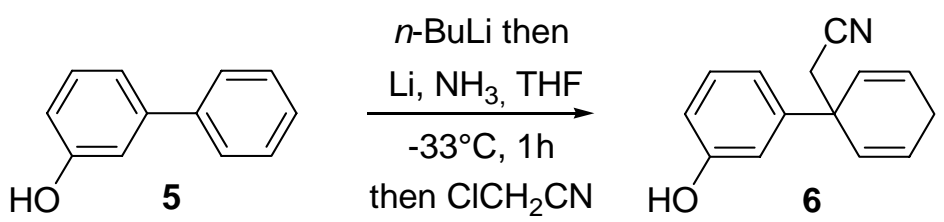

$68 \%$

[1-(3-Hydroxy-phenyl)-cyclohexa-2,5-dienyl]-acetonitrile (6). This product was obtained as a white solid (68 \%) and could be recrystallized in DCM/hexane. - m.p. 106.4-108.6 ${ }^{\circ} \mathrm{C}$. - IR (solid, $\mathrm{KBr}): v=3343 \mathrm{~cm}^{-1}, 2269(\mathrm{CN}), 1612,1586,1483,1330,1274,1230,840,789,738,700 .-{ }^{1} \mathrm{H}$ NMR $\left(\mathrm{CDCl}_{3}, 250 \mathrm{MHz}\right): \delta(\mathrm{ppm})=7.21(\mathrm{t}, J=8.2 \mathrm{~Hz}, 1 \mathrm{H}$, aromatic H), 6.86-6.69 (m, 3H aromatic H), 6.05-5.98 (m, 2H, vinylic H), 5.76-5.67 (m, 2H, vinylic H), 5.50 (broad s, OH), 2.88$2.65(\mathrm{~m}, 2 \mathrm{H}, \mathrm{CH} a \mathrm{Hb}), 2.82\left(\mathrm{~s}, 2 \mathrm{H}, \mathrm{CH}_{2} \mathrm{CN}\right) .{ }^{13} \mathrm{C} \mathrm{NMR}\left(\mathrm{CDCl}_{3}, 62.9 \mathrm{MHz}\right): \delta(\mathrm{ppm})=156.1(\mathrm{~s}$, aromatic C), 146.4 (s, aromatic H), 130.0 (d, aromatic $\mathrm{CH}$ ), 129.4 (d, 2 x vinylic $\mathrm{CH}$ ), 126.3 (d, 2 x vinylic $\mathrm{CH}$ ), 118.2 (d, aromatic $\mathrm{CH}), 117.9$ (s, CN), 114.2 (d, aromatic $\mathrm{CH}), 113.7$ (d, aromatic $\mathrm{CH}$ ), 42.3 (s, aliphatic C), 30.1 (t, $\underline{\mathrm{C}}_{2} \mathrm{CN}$ ), 26.1 (t, $\mathrm{CHaHb}$ ). - MS (SIMS) m/z (\%): 234 [M+Na] (100), $212[\mathrm{M}+\mathrm{H}]^{+}(50)$. - HRMS $[\mathrm{M}+\mathrm{Na}]^{+} \mathrm{C}_{14} \mathrm{H}_{13} \mathrm{NONa}$ : Calcd 234.0895; found: 234.0896. Anal. Calcd for $\mathrm{C}_{14} \mathrm{H}_{13} \mathrm{NO}$ (211.26): C 79.59, H 6.20, N 6.63; found: C 79.26, H 6.26, N 6.45.
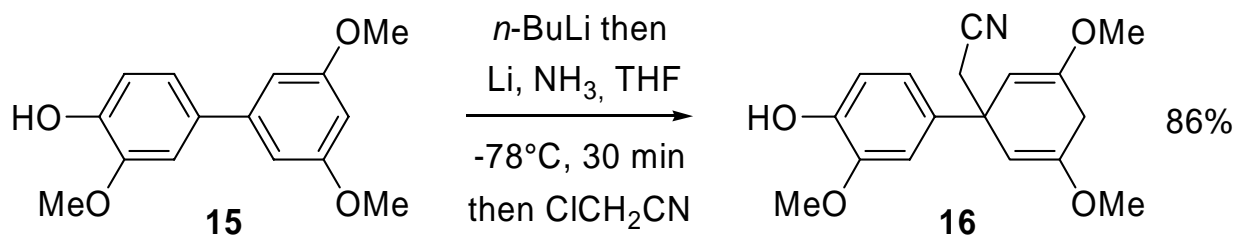

[1-(4-Hydroxy-3-methoxy-phenyl)-3,5-dimethoxy-cyclohexa-2,5-dienyl]-acetonitrile (16). This product was obtained as a viscous yellow oil (86\%). - IR (film, $\mathrm{NaCl}): v=3426 \mathrm{~cm}^{-1}, 2938,2250$ (CN), 1694, 1515, 1263, 1206, 1146, 1022, 806, 736. - ${ }^{1} \mathrm{H} \mathrm{NMR}\left(\mathrm{CDCl}_{3}, 300 \mathrm{MHz}\right): \delta=6.90-6.76$ (m, 3H, aromatic H), 5.73 (broad s, $1 \mathrm{H}, \mathrm{OH}), 4.69$ (s, 2H, 2 x vinylic $\mathrm{H}$ ), 3.87 (s, 3H, $\mathrm{OCH}_{3}$ ), 3.60 (s, 6H, $2 \times \mathrm{OCH}_{3}$ ), 2.91 (AB system, $\left.J_{a b}=24.5 \mathrm{~Hz}, 2 \mathrm{H}, \mathrm{CHaHb}\right), 2.89$ (s, 2H, $\mathrm{CH}_{2} \mathrm{CN}$ ). - ${ }^{13} \mathrm{C}$ NMR $\left(\mathrm{CDCl}_{3}, 62.9 \mathrm{MHz}\right): \delta=153.1$ (s, 2 x aromatic C), 146.5 (s, aromatic C), 144.5 (s, aromatic C), 138.5 (s, aromatic C), 118.3 (s, CN), 118,0 (d, aromatic CH), 114.2 (d, aromatic CH), 109.2 (d, aromatic $\mathrm{CH}$ ), 98.8 (d, $2 \mathrm{x}$ vinylic $\mathrm{CH}$ ), 56.0 (q, $\mathrm{OCH}_{3}$ ), 54.6 (q, $2 \times \mathrm{OCH}_{3}$ ), 44.7 (s, aliphatic C), 32.5 (t, $\mathrm{CH}_{2} \mathrm{CN}$ ), 31.1 (t, $\mathrm{CHaHb}$ ). - HRMS [M] ${ }^{+} \mathrm{C}_{17} \mathrm{H}_{19} \mathrm{NO}_{4}$ : calcd 301.1314; found 301.1310. 

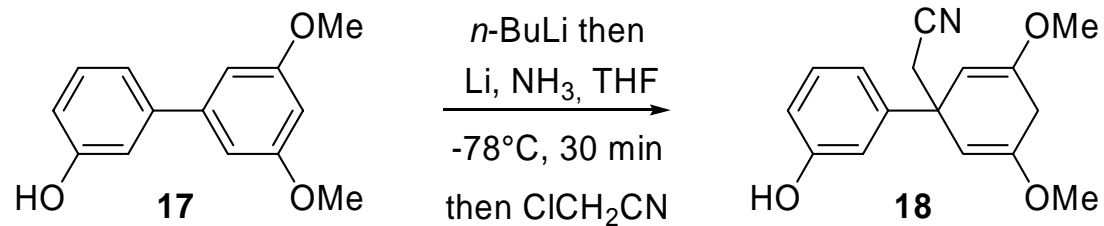

$75 \%$

[1-(3-Hydroxy-phenyl)-3,5-dimethoxy-cyclohexa-2,5-dienyl]-acetonitrile (18). This product was obtained as a white solid (75\%). - m.p. 154.6-155.3 ${ }^{\circ} \mathrm{C}$. - IR (solid, $\left.\mathrm{KBr}\right): v=3265 \mathrm{~cm}^{-1}(\mathrm{OH})$, 2946, 2272 (CN), 1694, 1586, 1403, 1245, 1208, 1156, 1022, 842, 790, 705. - ${ }^{1} \mathrm{H}$ NMR $\left(\mathrm{CDCl}_{3}\right.$, $250 \mathrm{MHz}): \delta=7.20(\mathrm{t}, J=7.9 \mathrm{~Hz}, 1 \mathrm{H}$, aromatic $\mathrm{H}), 6.88(\mathrm{~d}, J=7.6 \mathrm{~Hz}, 1 \mathrm{H}$, aromatic $\mathrm{H}), 6.81(\mathrm{t}, J$ $=1.5 \mathrm{~Hz}$, aromatic H), $6.69(\mathrm{dd}, J=1.5,7.9 \mathrm{~Hz}, 1 \mathrm{H}$, aromatic H), $5.43(\mathrm{~s}, 1 \mathrm{H}, \mathrm{OH}), 4.67(\mathrm{~s}, 2 \mathrm{H}, 2 \mathrm{x}$ vinylic H), 3.59 (s, 6H, $\left.2 \times \mathrm{OCH}_{3}\right), 2.99-2.80\left(\mathrm{~m}, 4 \mathrm{H}, \mathrm{CH}_{2} \mathrm{CN}\right.$ and $\left.\mathrm{CHaHb}\right) .{ }^{13} \mathrm{C} \mathrm{NMR}\left(\mathrm{CDCl}_{3}\right.$, $62.9 \mathrm{MHz}$ ): $\delta=156.0$ (s, aromatic C), 153.4 (s, 2 x aromatic C), 148.4 (s, aromatic C), 129.9 (d, aromatic $\mathrm{CH}$ ), 118.3 (s, CN), 117.8 (d, aromatic $\mathrm{CH}$ ), 113.9 (d, aromatic $\mathrm{CH}$ ), 113.5 (d, aromatic $\mathrm{CH}$ ), 98.4 (d, 2 x vinylic $\mathrm{CH}$ ), 54.7 (q, $2 \times \mathrm{OCH}_{3}$ ), 45.0 (s, aliphatic C), 32.3 (t, $\underline{\mathrm{CH}}_{2} \mathrm{CN}$ ), 31.2 (t, $\mathrm{CHaHb}$ ). - HRMS [M-CH$\left.{ }_{2} \mathrm{CN}\right]^{+} \mathrm{C}_{14} \mathrm{H}_{15} \mathrm{O}_{3}$ : calcd 231.10212; found 231.1023. - Anal. Calcd for $\mathrm{C}_{16} \mathrm{H}_{17} \mathrm{NO}_{3}$ (271.31): C 70.83, H 6.32, N 5.16; found: C 70.76, H 6.31, N 5.07.
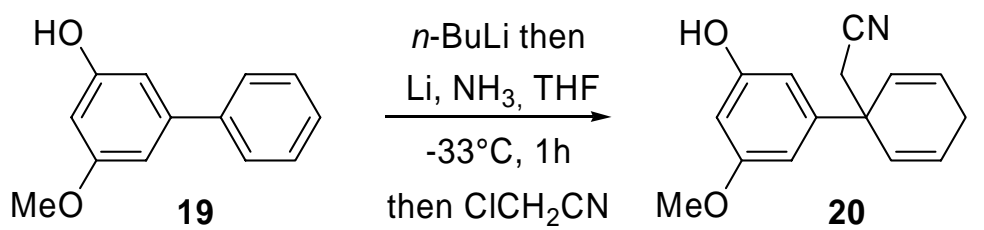

$48 \%$

[1-(3-Hydroxy-5-methoxy-phenyl)-cyclohexa-2,5-dienyl]-acetonitrile (20). This product was obtained as a white solid (48\%). - m.p. $=94.8-95.4^{\circ} \mathrm{C}$. - IR (solid, $\mathrm{KBr}$ ): $v=3419 \mathrm{~cm}^{-1}, 3302,2360$ (CN), 2342, 1598, 1163, 802, 668. - ${ }^{1} \mathrm{H} \mathrm{NMR}\left(\mathrm{CDCl}_{3}, 250 \mathrm{MHz}\right): \delta=6.41$ (s, $1 \mathrm{H}$, aromatic $\left.\mathrm{H}\right)$, 6.36 (s, $1 \mathrm{H}$, aromatic H), 6.29 (s, $1 \mathrm{H}$, aromatic $\mathrm{H}), 6.03-5.97$ (m, $2 \mathrm{H}, 2$ x vinylic $\mathrm{H}), 5.72-5.67$ (d, $J$ $=10.4 \mathrm{~Hz}, 2 \mathrm{H}, 2 \mathrm{x}$ vinylic $\mathrm{H}), 5.51$ (broad s, $1 \mathrm{H}, \mathrm{OH}), 3.75\left(\mathrm{~s}, 3 \mathrm{H}, \mathrm{OCH}_{3}\right), 2.87-2.40(\mathrm{~m}, 2 \mathrm{H}$, $\mathrm{CHaHb}), 2.78\left(\mathrm{~s}, 2 \mathrm{H}, \mathrm{CH}_{2} \mathrm{CN}\right) .-{ }^{13} \mathrm{C} \mathrm{NMR}\left(\mathrm{CDCl}_{3}, 62.9 \mathrm{MHz}\right): \delta=161.2$ (s, aromatic C), 157.2 (s, aromatic C), 147.3(s, aromatic C), 129.3(d, 2 x vinylic $\mathrm{CH}$ ), 126.3 (d, 2 x vinylic $\mathrm{CH}$ ), 117.9 (s, $\mathrm{CN}), 106.0$ (d, aromatic $\mathrm{CH}), 105.3$ (d, aromatic $\mathrm{CH}), 99.7$ (d, aromatic $\mathrm{CH}), 55.5$ (q, $\mathrm{OCH}_{3}$ ), 42.3 (s, aliphatic C), 30.1(t, $\underline{\mathrm{CH}}_{2} \mathrm{CN}$ ), 26.1 (t, $\mathrm{CHaHb}$ ). - MS (ESI) m/z (\%): $242[\mathrm{M}+\mathrm{H}]^{+}$(65), 201 [M$\left.\mathrm{CH}_{2} \mathrm{CN}\right]^{+}$(100). - HRMS [M] ${ }^{+\bullet} \mathrm{C}_{15} \mathrm{H}_{15} \mathrm{NO}_{2}$ : Calcd 241.11028; found: 241.1110.
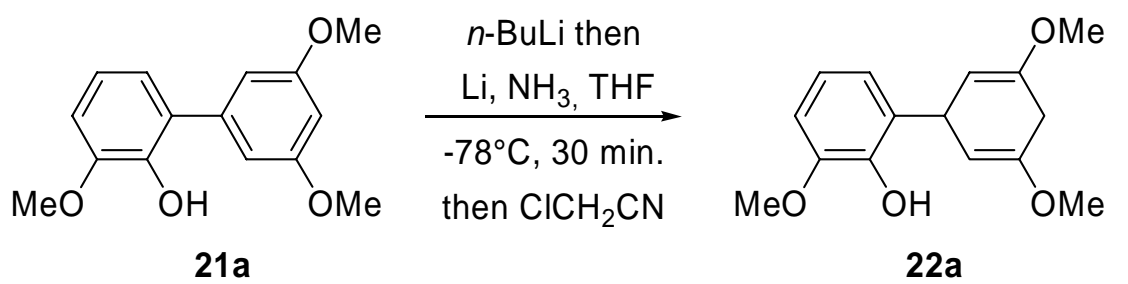

$82 \%$

2-(3,5-Dimethoxy-cyclohexa-2,5-dienyl)-6-methoxy-phenol (22a). This product was obtained as a white solid (82\%) but showed a strong tendency for rearomatization. - IR (solid, $\mathrm{KBr}$ ): $v=3377$ $\mathrm{cm}^{-1}, 2938,2837,1696,1480,1273,1228,1206,1145,1070,735 .{ }^{1}{ }^{\mathrm{H}} \mathrm{NMR}\left(\mathrm{CDCl}_{3}, 300 \mathrm{MHz}\right): \delta$ $=6.84-6.86(\mathrm{~m}, 2 \mathrm{H}$, aromatic $\mathrm{H}), 6.79-6.75(\mathrm{~m}, 1 \mathrm{H}$, aromatic $\mathrm{H}), 5.93(\mathrm{~s}, 1 \mathrm{H}, \mathrm{OH}), 4.80(\mathrm{~d}, J=3.8$ $\mathrm{Hz}, 2$ x vinylic $\mathrm{CH}$ ), 4.76-4.69 (m, 1H, CH), 3.91 (s, 3H, $\left.\mathrm{OCH}_{3}\right), 3.59$ (s, 6H, 2 x OCH $\mathrm{OCH}_{3}$, 3.00-2.83 (m, $2 \mathrm{H}, \mathrm{CHaHb}) .{ }^{13} \mathrm{C} \mathrm{NMR}\left(\mathrm{CDCl}_{3}, 75.5 \mathrm{MHz}\right): \delta=152.0$ (s, 2 x vinylic C), 146.4 (s, aromatic C), 142.8 (s, aromatic C), 132.1 (s, aromatic C), 120.8 (d, aromatic $\mathrm{CH}$ ), 119.6 (d, aromatic $\mathrm{CH}$ ), 108.5 (d, aromatic $\mathrm{CH}$ ), 95.3 (d, 2 x vinylic $\mathrm{CH}$ ), 56.1 (q, $\left.\mathrm{OCH}_{3}\right), 54.3$ (q, $\left.2 \mathrm{x} \mathrm{OCH}_{3}\right), 35.0(\mathrm{~d}, \mathrm{CH})$, 31.1 (t, $\mathrm{CH} a \mathrm{Hb}$ ). - HRMS [M] ${ }^{+} \mathrm{C}_{15} \mathrm{H}_{18} \mathrm{O}_{4}$ : calcd 262.12051; found 262.1201. 

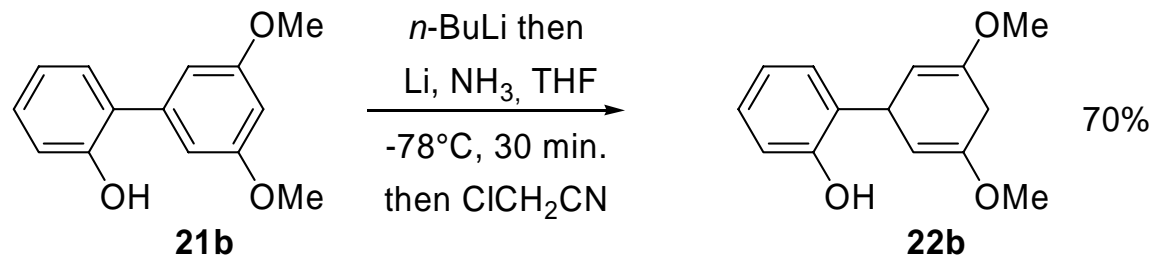

2-(3,5-Dimethoxy-cyclohexa-2,5-dienyl)-phenol (22b). This compound has been isolated by chromatography with degased solvents but is really sensitive to oxygen as it rearomatizes quickly. For that purpose, we were unable to get MS (or HRMS) analysis. - IR (solid, KBr): $v=3441 \mathrm{~cm}^{-1}$, 2960, 1694, 1452, 1400, 1271, 1210, 1143, 854, 748. - ${ }^{1} \mathrm{H} \mathrm{NMR}\left(\mathrm{CDCl}_{3}, 250 \mathrm{MHz}\right): \delta=7.16-7.13$ (m, 2H, aromatic H), 6.91-6.80 (m, 2H, aromatic H), 5.69 (broad s, $1 \mathrm{H}, \mathrm{OH}), 4.77$ (d, $J=2.5 \mathrm{~Hz}$, $2 \mathrm{H}, 2$ x vinylic $\mathrm{H}$ ), 3.78 (m, 1H, aliphatic $\mathrm{CH}$ ), 3.58 (s, 6H, 2 x $\mathrm{OCH}_{3}$ ), 3.01-2.83 (d, $J=7.6 \mathrm{~Hz}$, $2 \mathrm{H}, \mathrm{CH} a \mathrm{Hb}$ ). ${ }^{13} \mathrm{C} \mathrm{NMR}\left(\mathrm{CDCl}_{3}, 62.9 \mathrm{MHz}\right.$ ): $\delta=155.1$ (s, CqAr-OH), 153.0 (s, Cq-OMe), 130.2 (s, aromatic C), 129.4 (d, aromatic $\mathrm{C}$ meta $\mathrm{OH}), 128.2$ (d, aromatic $\mathrm{C}$ meta $\mathrm{OH}$ ), 120.6 (d, aromatic C para $\mathrm{OH}$ ), 116.6 (d, aromatic $\mathrm{C}$ ortho $\mathrm{OH}), 94.6$ (d, 2 x vinylic $\mathrm{CH}$ ), 54.6 (q, 2 x $\mathrm{OCH}_{3}$ ), 39.6 (d, benzylic $\mathrm{CH}), 31.1$ (t, $\mathrm{CH} a \mathrm{H} b$ ).

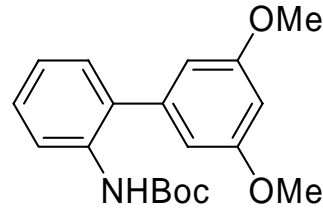

23

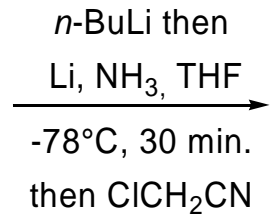

then $\mathrm{ClCH}_{2} \mathrm{CN}$

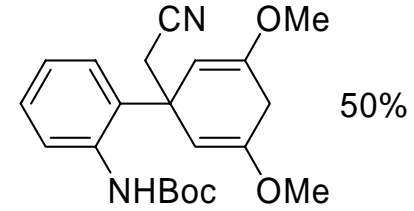

24

[2-(1-Cyanomethyl-3,5-dimethoxy-cyclohexa-2,5-dienyl)-phenyl]-carbamic acid tert-butyl ester (24). This product was obtained as a white solid (50\%). - m.p. 158.2-158.4 ${ }^{\circ} \mathrm{C}$. - IR (solid, $\mathrm{KBr}): v=3412 \mathrm{~cm}^{-1}, 2933,1722,1686,1510,1446,1208,1156,1022,876,771,760 .-{ }^{1} \mathrm{H}$ NMR $\left(\mathrm{CDCl}_{3}, 300 \mathrm{MHz}\right): \delta=7.67(\mathrm{dd}, J=1.1,7.9 \mathrm{~Hz}, 1 \mathrm{H}$, aromatic $\mathrm{H}), 7.34-7.28(\mathrm{~m}, 1 \mathrm{H}$, aromatic $\mathrm{H})$, 7.19-7.08 (m, 2H, aromatic H), 6.94 (broad s, 1H, NH), 4.55 (s, 2H, 2 x vinylic H ), 3.57 (s, 6H, 2 x $\left.\mathrm{OCH}_{3}\right), 3.12(\mathrm{AB}$ system, $J=21.1 \mathrm{~Hz}, 1 \mathrm{H}, \mathrm{CH} a \mathrm{CH} b), 2.99$ (AB system, $J=21.1 \mathrm{~Hz}, 1 \mathrm{H}$,

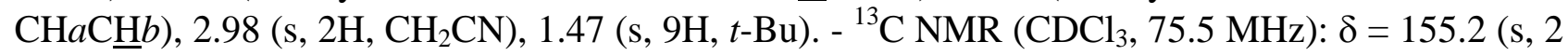
x vinylic C), 153.2 (s, aromatic C), 137.4 (s, aromatic C), 133.9 (s, aromatic C), 128.4 (d, aromatic $\mathrm{CH}), 125.3$ (d, aromatic $\mathrm{CH}), 124.1$ (d, aromatic $\mathrm{CH}), 124.0$ (d, aromatic $\mathrm{C}$ ), 117.8 (s, aromatic C), 96.7 (d, 2 x vinylic $\mathrm{CH}$ ), 80.2 (s, $\left.\underline{\mathrm{C}}\left(\mathrm{CH}_{3}\right)_{3}\right) .54 .9$ (q, 2 x $\left.\mathrm{OCH}_{3}\right), 43.8$ (s, aliphatic C), 33.2 (t, $\mathrm{CH}_{2} \mathrm{CN}$ ), 31.3 (t, $\mathrm{CHaHb}$ ), $28.3\left(\mathrm{q}, 3 \times \mathrm{CH}_{3}\right)$. - HRMS [M- $\left.\mathrm{CH}_{2} \mathrm{CN}-\mathrm{C}_{4} \mathrm{H}_{8}\right]^{+} \mathrm{C}_{15} \mathrm{H}_{16} \mathrm{NO}_{4}$ : calcd 274.10793; found 274.1078; [M-CH$\left.-\mathrm{CHN}_{2} \mathrm{C}_{4} \mathrm{H}_{8}-\mathrm{CO}_{2}\right]^{+} \mathrm{C}_{14} \mathrm{H}_{16} \mathrm{NO}_{2}$ : calcd 230.11810; found 230.1181.

\section{E) Experimental procedures for crinine analog synthesis:}<smiles>COc1cc(O)cc(C2(CC#N)C=CCC=C2)c1</smiles>
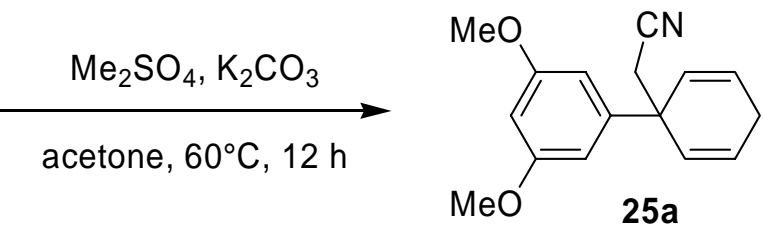

$95 \%$

[1-(3,5-Dimethoxyphenyl)-cyclohexa-2,5-dienyl]-acetonitrile (25a). In a dry two-necked flask was introduced [1-(3-Hydroxy-5-methoxy-phenyl)-cyclohexa-2,5-dienyl]-acetonitrile (1,144 g, 4.75 mmol, 1 eq.) and potassium carbonate $(9.83 \mathrm{~g}, 71 \mathrm{mmol}, 15$ eq.) in acetone (50 mL). Dimethyl 
sulfate ( $4.5 \mathrm{~mL}, 47 \mathrm{mmol}, 10$ eq.) was added via a syringe and the media was carried to reflux overnight. Acetone was evaporated and water was added. Extraction with ether, washing with brine, drying with sodium sulfate and evaporation of the solvent gave an oily product, which could be purified by silica gel chromatography (Petroleum ether/EtOAc, 9:1). The title compound was obtained as a yellow oil (1.07 g, $4.18 \mathrm{mmol}, 88 \%)$. - IR (film, $\mathrm{NaCl}): v=2961 \mathrm{~cm}^{-1}, 2873,2250$ (CN), 1594, 1457, 1425, 1311, 1205, 1157, 1064, 836, 730, 693. - ${ }^{1} \mathrm{H}$ NMR $\left(\mathrm{CDCl}_{3}, 250 \mathrm{MHz}\right): \delta=$ $6.44(\mathrm{~d}, J=2.2 \mathrm{~Hz}, 2 \mathrm{H}, 2 \mathrm{x}$ aromatic H), $6.34(\mathrm{t}, J=2.2 \mathrm{~Hz}, 1 \mathrm{H}$, aromatic $\mathrm{H}), 6.05-5.98(\mathrm{~m}, 2 \mathrm{H}, 2 \mathrm{x}$ vinylic $\mathrm{H}$ ), 5.75-5.69 (m, $2 \mathrm{H}, 2 \mathrm{x}$ vinylic $\mathrm{H}), 3.78$ (s, $\left.6 \mathrm{H}, 2 \times \mathrm{OCH}_{3}\right), 2.89-2.64(\mathrm{~m}, 2 \mathrm{H}, \mathrm{CH} a \mathrm{Hb})$, 2.81 (t, 2H, $\left.\mathrm{CH}_{2} \mathrm{CN}\right) .{ }^{13} \mathrm{C} \mathrm{NMR}\left(\mathrm{CDCl}_{3}, 62.9 \mathrm{MHz}\right): \delta=161.1$ (s, $2 \mathrm{x}$ aromatic C), 147.0 (s, aromatic C), 129.4 (d, 2 x vinylic $\mathrm{CH}$ ), 126.2 (d, 2 x vinylic $\mathrm{CH}$ ), 117.7 (s, CN), 104.9 (d, 2 x aromatic $\mathrm{CH}$ ), 98.2 (d, aromatic $\mathrm{CH}$ ), 55.4 (q, $2 \times \mathrm{OCH}_{3}$ ), 42.4 (s, aliphatic $\mathrm{C}$ ), 30.1 (t, $\mathrm{CH}_{2} \mathrm{CN}$ ), 26.0 (t, $\mathrm{CH} a \mathrm{H} b$ ). - HRMS [M] ${ }^{+} \mathrm{C}_{16} \mathrm{H}_{17} \mathrm{NO}_{2}$ : calcd 255.12593; found 255.1252.
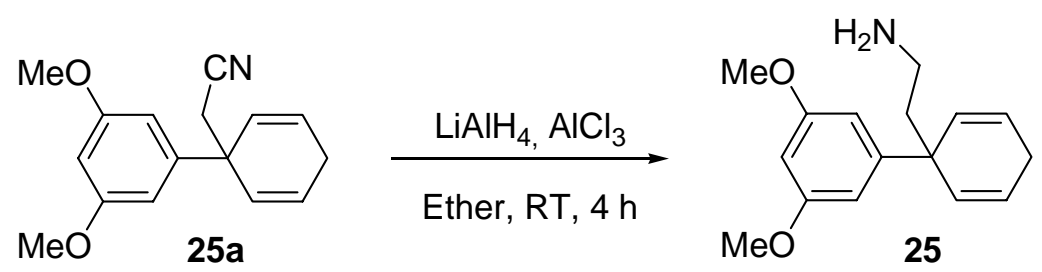

$78 \%$

2-[1-(3,5-Dimethoxyphenyl)-cyclohexa-2,5-dienyl]-ethylamine (25). In a two-necked flask was introduced $\mathrm{AlCl}_{3}(0.613 \mathrm{~g}, 4.60 \mathrm{mmol})$ in ether $(40 \mathrm{~mL})$ at $0{ }^{\circ} \mathrm{C}$. A solution of $\mathrm{LiAlH}_{4}(1 \mathrm{M}$ in ether, $4.6 \mathrm{~mL}, 4.60 \mathrm{mmol}$ ) was added dropwise and the mixture was allowed to stirr for 30 minutes at room temperature. [1-(3,5-Dimethoxy-phenyl)-cyclohexa-2,5-dienyl]-acetonitrile (1.066 g, 4.18 mmol) was diluted in ether $(40 \mathrm{~mL})$ and added dropwise at $0{ }^{\circ} \mathrm{C}$. The media was stirred at room temperature for 4 hours. Water $(2 \mathrm{~mL})$ was cautiously added followed by a $10 \%$ sodium hydroxide solution and the media was stirred for 15 minutes. Filtration of the solid residue over celite, washing generously with ether, separation of the two layers, drying of the organic layer over sodium sulfate and evaporation of the solvent gave an oily product. The product was in general sufficiently pure to be used in the next step without purification. An analytically pure sample was obtained after purification by silica gel (deactivated with $5 \% \mathrm{Et}_{3} \mathrm{~N}$ ) chromatography (DCM/MeOH, 98:2) to give a colorless oil $(0.840 \mathrm{~g}, 3.24 \mathrm{mmol}, 78 \%)$. IR (film, NaCl): $v=3366 \mathrm{~cm}^{-1}, 2936$, 1594, 1455, 1424, 1307, 1204, 1155, 1062, 836, 794, 739, 694. - ${ }^{1} \mathrm{H}$ NMR $\left(\mathrm{CDCl}_{3}, 300 \mathrm{MHz}\right): \delta=$ $6.52(\mathrm{~d}, J=2.3 \mathrm{~Hz}, 2 \mathrm{H}, 2 \mathrm{x}$ aromatic $\mathrm{H}), 6.29(\mathrm{t}, J=2.3 \mathrm{~Hz}, 1 \mathrm{H}$, aromatic H), 5.94-5.78 (m, $2 \mathrm{H}, 2 \mathrm{x}$ vinylic $\mathrm{H}$ ), 6.64-5.58 (m, $2 \mathrm{H}, 2 \mathrm{x}$ vinylic $\mathrm{H}$ ), 3.76 (s, $\left.6 \mathrm{H}, 2 \times \mathrm{OCH}_{3}\right), 2.74-2.65\left(\mathrm{~m}, 4 \mathrm{H}, \mathrm{C}_{2} \mathrm{NH}_{2}\right.$ and $\mathrm{CHaHb}$ ), 196-1.90 (m, 2H, $\underline{\mathrm{C}}_{2} \mathrm{CH}_{2} \mathrm{NH}_{2}$ ), 1.36 (broad s, $2 \mathrm{H}, \mathrm{NH}_{2}$ ). $-{ }^{13} \mathrm{C} \mathrm{NMR}\left(\mathrm{CDCl}_{3}, 75.5\right.$ $\mathrm{MHz}$ ): $\delta=160.8$ (s, $2 \mathrm{x}$ aromatic C), 150.7 (s, aromatic C), 132.3 (d, 2 x vinylic $\mathrm{CH}$ ), 123.8 (d, $2 \mathrm{x}$ vinylic $\mathrm{CH}$ ), 105.1 (d, $2 \mathrm{x}$ aromatic $\mathrm{CH}), 97.6$ (d, aromatic $\mathrm{CH}), 55.3(\mathrm{q}, 2 \mathrm{x} \mathrm{OCH}), 44.0$ (t, $\mathrm{CH}_{2} \mathrm{CH}_{2} \mathrm{NH}_{2}$ ), 43.4 (s, aliphatic C), $39.0\left(\mathrm{t}, \mathrm{CH}_{2} \mathrm{NH}_{2}\right), 26.1$ (t, $\left.\mathrm{CHaHb}\right)$. $-\mathrm{HRMS}[\mathrm{M}]^{+}{ }^{\circ} \mathrm{C}_{16} \mathrm{H}_{21} \mathrm{NO}_{2}$ : calcd 259.15723; found 259.1572.
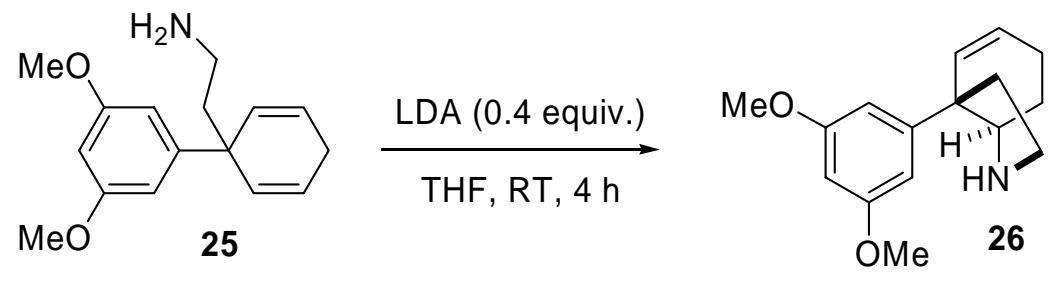

$73 \%$

3a-(3,5-Dimethoxy-phenyl)-2,3,3a,6,7,7a-hexahydro-1H-indole (26). To a stirred solution of 2[1-(3,5-dimethoxy-phenyl)-cyclohexa-2,5-dienyl]-ethylamine $(0.164 \mathrm{~g}, 0.63 \mathrm{mmol})$ diluted in THF (3 mL) was added dropwise a $0.5 \underline{\mathrm{M}}$ solution of lithium diisopropyl amine in THF $(0.50 \mathrm{~mL}, 0.25$ 
mmol) at room temperature. A dark red coloration appeared and the solution was strirred for 4 hours. A $10 \%$ sodium hydroxide solution was added and the aqueous layer was extracted with ether. The organic layer was directly dried over sodium sulfate to gave a paste which could be purified by silica gel (deactivated with $5 \% \mathrm{Et}_{3} \mathrm{~N}$ ) chromatography (DCM/MeOH, 98:2) to give 26 as an oily product (0.120 g, $0.46 \mathrm{mmol}, 73 \%$ ). - IR (film, $\mathrm{NaCl}): v=2934 \mathrm{~cm}^{-1}, 1595,1455,1422$, 1204, 1152, 1063, 835. - ${ }^{1} \mathrm{H}$ NMR $\left(\mathrm{CDCl}_{3}, 300 \mathrm{MHz}\right): \delta=6.52$ (d, $J=1.9 \mathrm{~Hz}, 2 \mathrm{H}$, aromatic H), $6.31(\mathrm{t}, J=1.9 \mathrm{~Hz}, 1 \mathrm{H}$, aromatic $\mathrm{H}), 5.81-5.74(\mathrm{~m}, 1 \mathrm{H}$, vinylic $\mathrm{H}), 5.65-5.59(\mathrm{~m}, 1 \mathrm{H}$, vinylic $\mathrm{H})$, 3.77 (s, 6H, $\left.2 \times \mathrm{OCH}_{3}\right), 3.24-3.07\left(\mathrm{~m}, 3 \mathrm{H}, \mathrm{CH}_{2} \mathrm{NH}\right.$ and $\left.\mathrm{CHNH}\right), 2.56-2.12(\mathrm{~m}, 7 \mathrm{H}$, aliphatic $\mathrm{H})$, 2.00-1.91 (m, $1 \mathrm{H}$, aliphatic H). ${ }^{13} \mathrm{C} \mathrm{NMR}\left(\mathrm{CDCl}_{3}, 75.5 \mathrm{MHz}\right): \delta=106.7$ (s, 2 x aromatic C), 148.6 (s, aromatic C), 125.3 (d, vinylic $\mathrm{CH}$ ), 124.0 (d, vinylic $\mathrm{CH}$ ), 105.0 (q, 2 x aromatic $\mathrm{CH}$ ), 97.3 (d, aromatic $\mathrm{CH}$ ), $61.8(\mathrm{~d}, \mathrm{CH}), 55.3\left(\mathrm{q}, 2 \times \mathrm{OCH}_{3}\right), 45.6$ (s, aliphatic $\left.\mathrm{C}\right), 43.1\left(\mathrm{t}, \mathrm{CH}_{2}\right), 40.6\left(\mathrm{CH}_{2}\right)$, 32.4 (t, $\mathrm{CH}_{2}$ ), 26.4 (t, $\mathrm{CH}_{2}$ ). - HRMS [M] ${ }^{+} \mathrm{C}_{16} \mathrm{H}_{21} \mathrm{NO}_{2}$ : calcd 259.15723; found 259.1577.
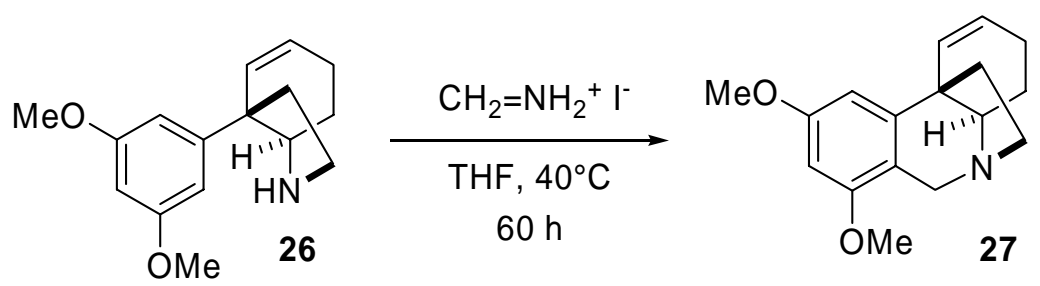

$82 \%$

Compound 27. In a two necked flask was introduced 3a-(3,5-dimethoxy-phenyl)-2,3,3a,6,7,7ahexahydro- $1 H$-indole 26 (0.085 g, $0.33 \mathrm{mmol})$, in THF $(16 \mathrm{~mL})$ and Eschenmoser's salt $(0.091 \mathrm{~g}$, $0.49 \mathrm{mmol}$ ). The solution was refluxed for 60 hours. After evaporation of THF, a $10 \%$ sodium hydroxide solution was added and the aqueous layer was extracted with ether. The organic layer was washed with brine, dried over sodium sulfate and evaporated. The crude paste was purified by silica gel (deactivated with $5 \% \mathrm{Et}_{3} \mathrm{~N}$ ) chromatography (Petroleum ether/THF, 3:1) to give the crinine squeletton 27 (0.071, $0.26 \mathrm{mmol}, 81 \%)$.

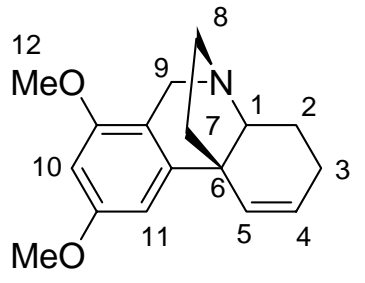

13 - IR (solid, KBr): $v=2924 \mathrm{~cm}^{-1}, 2879,1594,1463,1430,1307,1200,1144$, 1056, 840, 735, 680. - ${ }^{1} \mathrm{H}$ NMR $\left(\mathrm{CDCl}_{3}, 300 \mathrm{MHz}\right): \delta=6.37$ (d, $J=2.2 \mathrm{~Hz}$, $1 \mathrm{H}$, aromatic $\mathrm{H}), 6.28(\mathrm{~d}, J=2.2 \mathrm{~Hz}, 1 \mathrm{H}$, aromatic $\mathrm{H}), 5.72-5.64(\mathrm{~m}, 2 \mathrm{H}$, vinylic H), 4.14 (AB system, $J=17.3 \mathrm{~Hz}, 1 \mathrm{H}, \mathrm{Ar}-\mathrm{C} \underline{\mathrm{H}} a \mathrm{H} b-\mathrm{N} \mathrm{H9}$ ), 3.80 (s, $3 \mathrm{H}, \mathrm{OCH}_{3}$ ), $3.78(\mathrm{AB}$ system, $J=17.3 \mathrm{~Hz}, 1 \mathrm{H}, \mathrm{Ar}-\mathrm{CH} a \underline{\mathrm{H}} b-\mathrm{N} \mathrm{H9}$ ), 3.76 (s, $3 \mathrm{H}, \mathrm{OCH}_{3}$ ), 3.42-3.33 (m, $\left.1 \mathrm{H}, \mathrm{C} \underline{\mathrm{H}} a \mathrm{H} b \mathrm{~N} \mathrm{H8}\right), 3.11$ (t, $J=8.3 \mathrm{~Hz}, 1 \mathrm{H}, \mathrm{CHN}$ $\mathrm{H} 1$ ), 2.82-2.67 (m, 2H, allylic $\mathrm{CH}$ and $\mathrm{Ch} a \underline{\mathrm{H}} b \mathrm{~N} \mathrm{H3}$ and $\mathrm{H} 8$ ), 2.55-2.48 (m, 1H, allylic CH H3), 2.40-2.31 (m, 1H, Cㅍ $a \mathrm{H} b \mathrm{CHN} \mathrm{H} 2), 2.14-2.04$ (m, 1H, $\left.\mathrm{CH} a \mathrm{H} b-\mathrm{CH}_{2} \mathrm{~N} \mathrm{H7}\right), 1.99-1.88$ (m, $2 \mathrm{H}, \mathrm{CH} a \underline{\mathrm{H}} b \mathrm{CHN}$ and $\mathrm{CH} a \underline{\mathrm{H}} b-\mathrm{CH}_{2} \mathrm{~N} \mathrm{H} 2$ and H7). - ${ }^{13} \mathrm{C}$ NMR $\left(\mathrm{CDCl}_{3}, 75.5 \mathrm{MHz}\right.$ ): $\delta=159.5$ (s, aromatic C), 157.7 (s, aromatic C), 149.7 (s, aromatic C), 124.8 (d, vinylic $\mathrm{CH}$ ), 124.3 (d, vinylic $\mathrm{CH}$ ), 113.6 (s, aromatic $\mathrm{C}$ ), 100.0 (d, aromatic $\mathrm{CH}$ ), 95.4 (d, aromatic C), $62.3(\mathrm{~d}, \mathrm{CHN}), 57.4\left(\mathrm{t}, \mathrm{ArCH}_{2} \mathrm{~N} \mathrm{C9}\right), 55.4$ (q, $\left.\mathrm{OCH}_{3}\right), 55.2$ (q, $\left.\mathrm{OCH}_{3}\right), 51.6$ (t, $\mathrm{CH}_{2} \mathrm{CH}_{2} \mathrm{~N}$ ), 41.5 (s, aliphatic C C6), $40.6\left(\mathrm{t}, \underline{\mathrm{CH}}_{2} \mathrm{CH}_{2} \mathrm{~N}\right), 30.2$ (t, allylic $\mathrm{CH}_{2} \mathrm{C} 3$ ), 27.8 (t, $\mathrm{CH}_{2} \mathrm{CHN}$ ). - HRMS [M] ${ }^{+} \mathrm{C}_{17} \mathrm{H}_{21} \mathrm{NO}_{2}$ : calcd 271.15723; found 271.1584. 


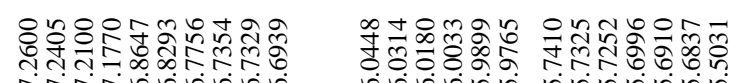
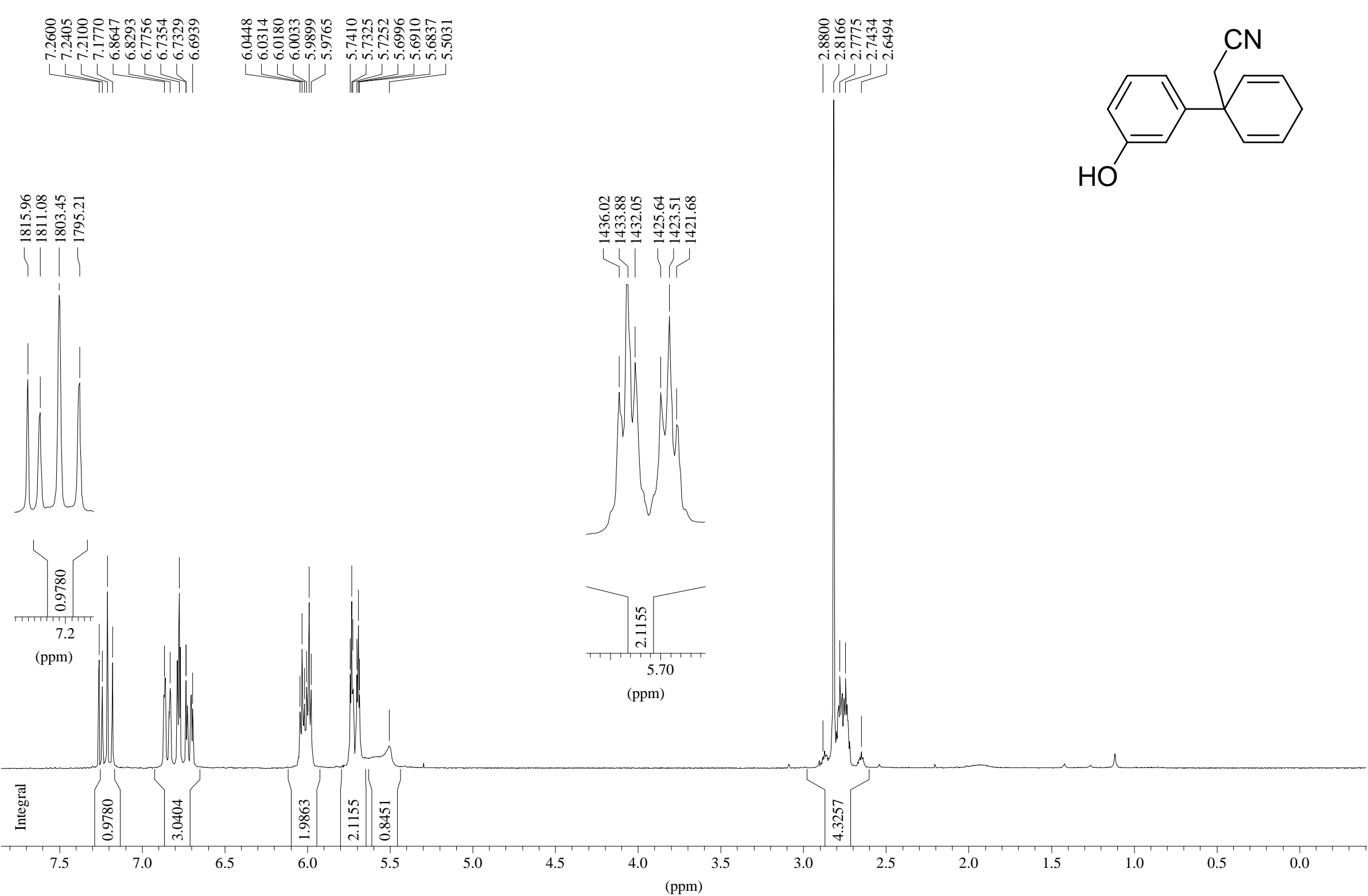


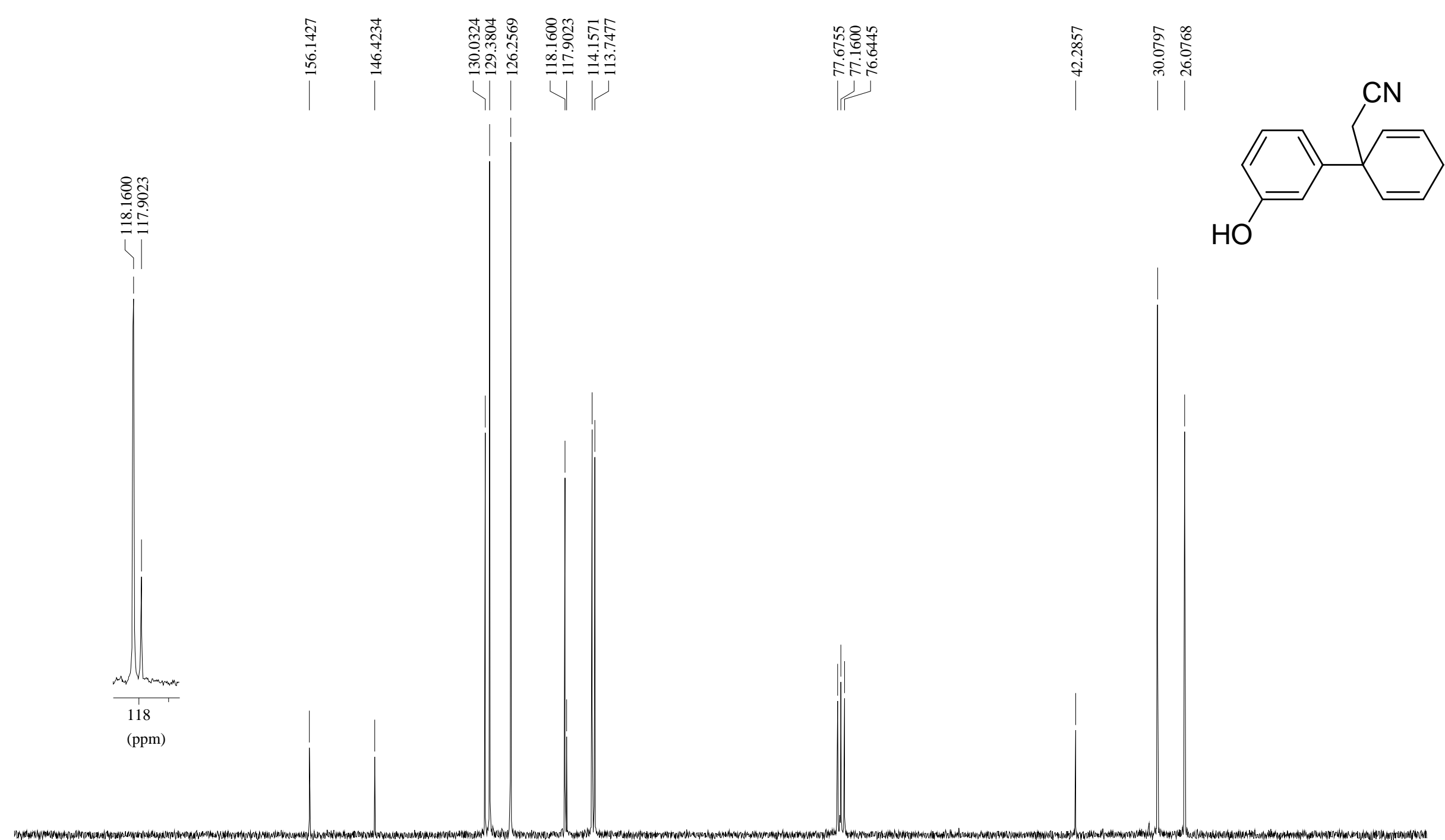




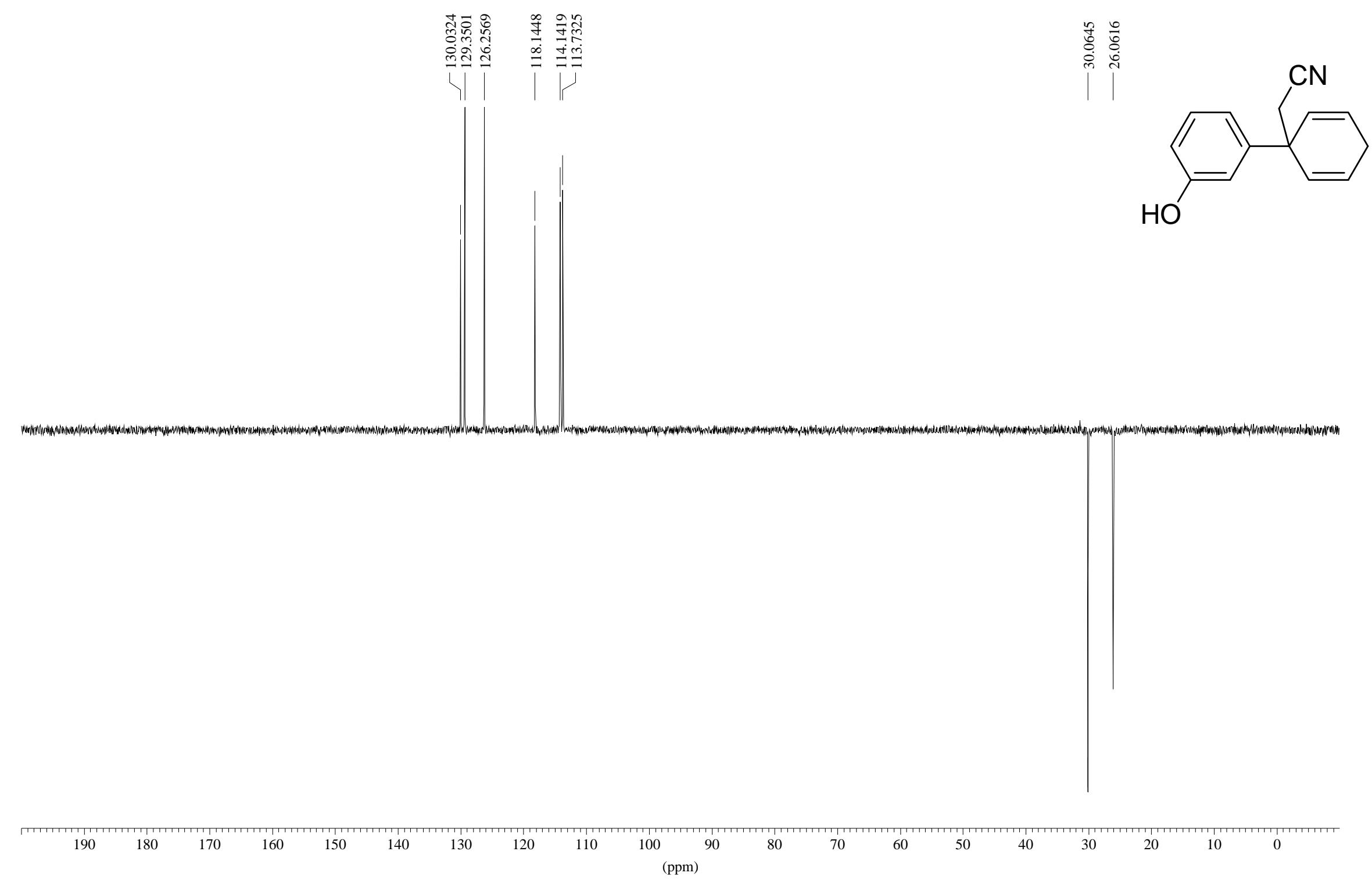




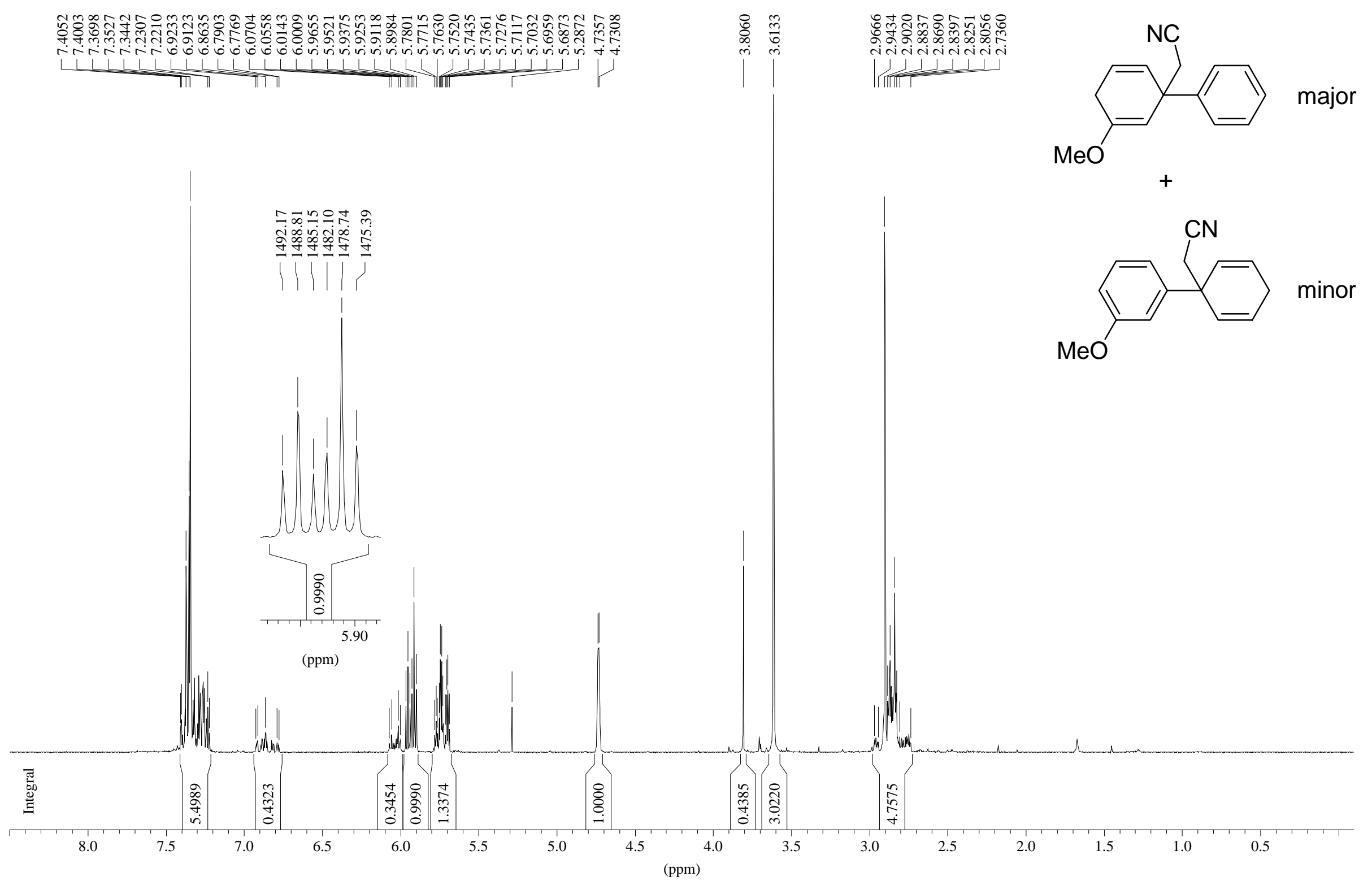



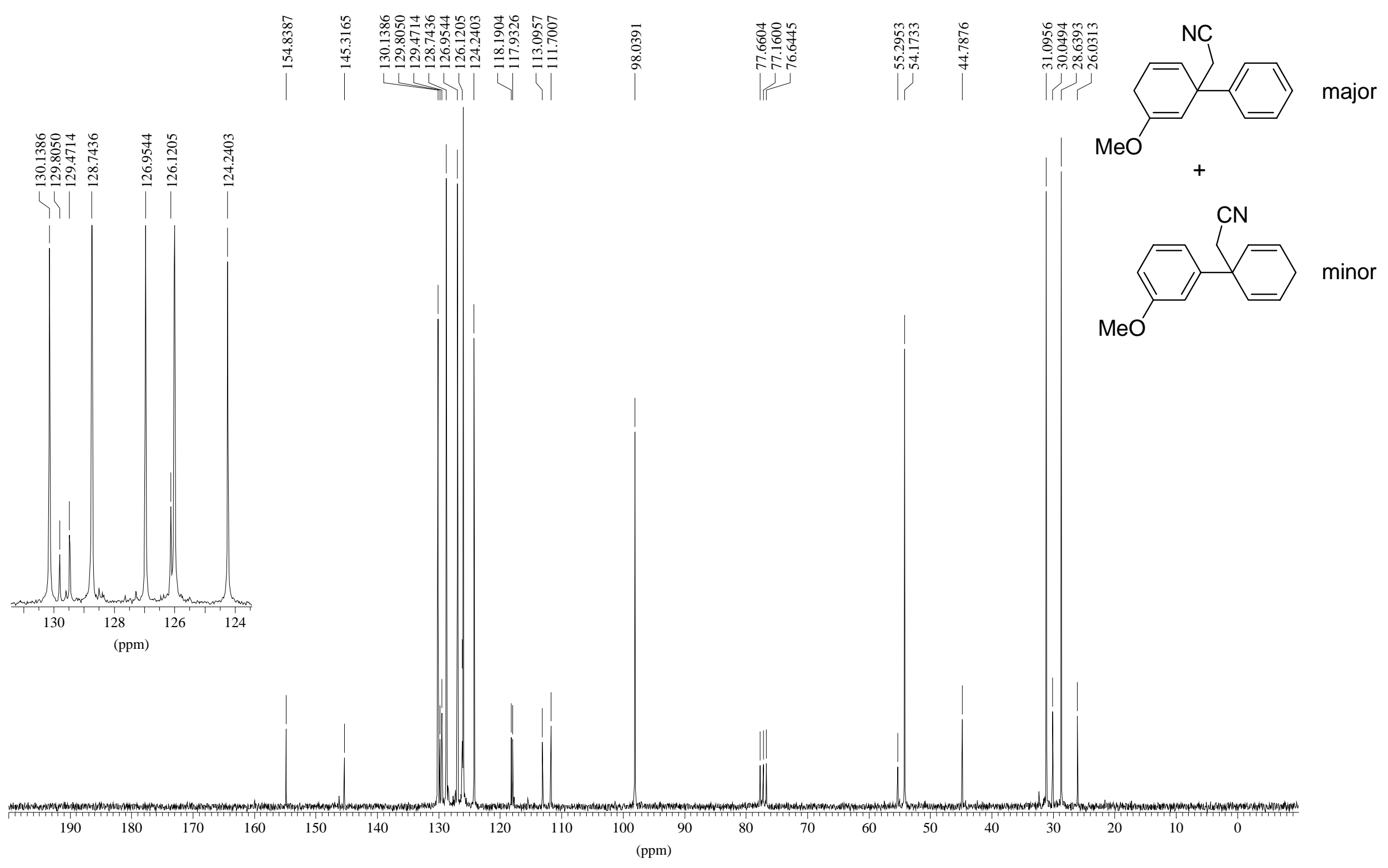

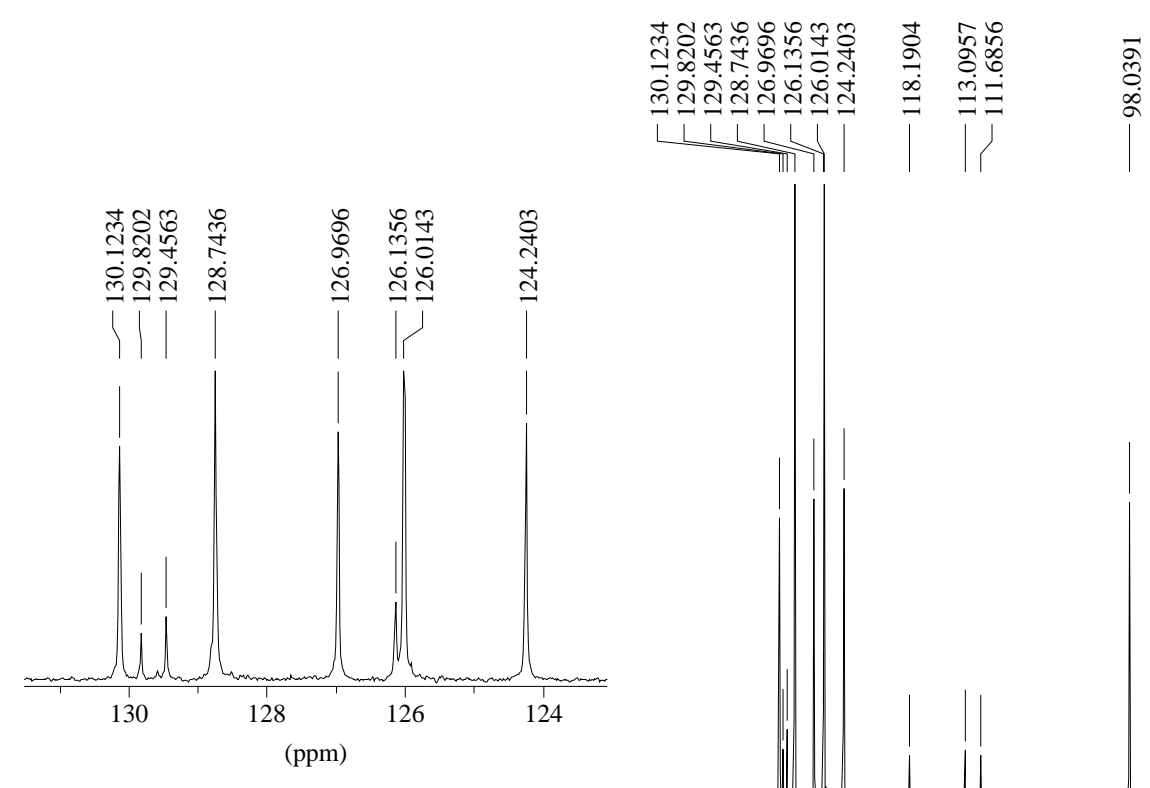

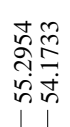
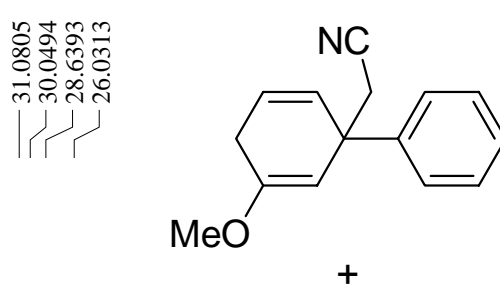

major $\mathrm{MeO}$

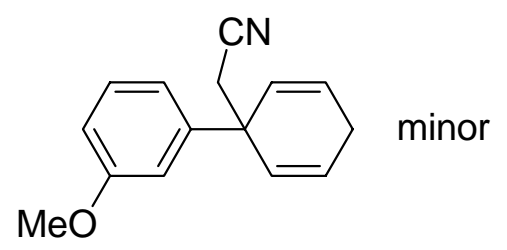




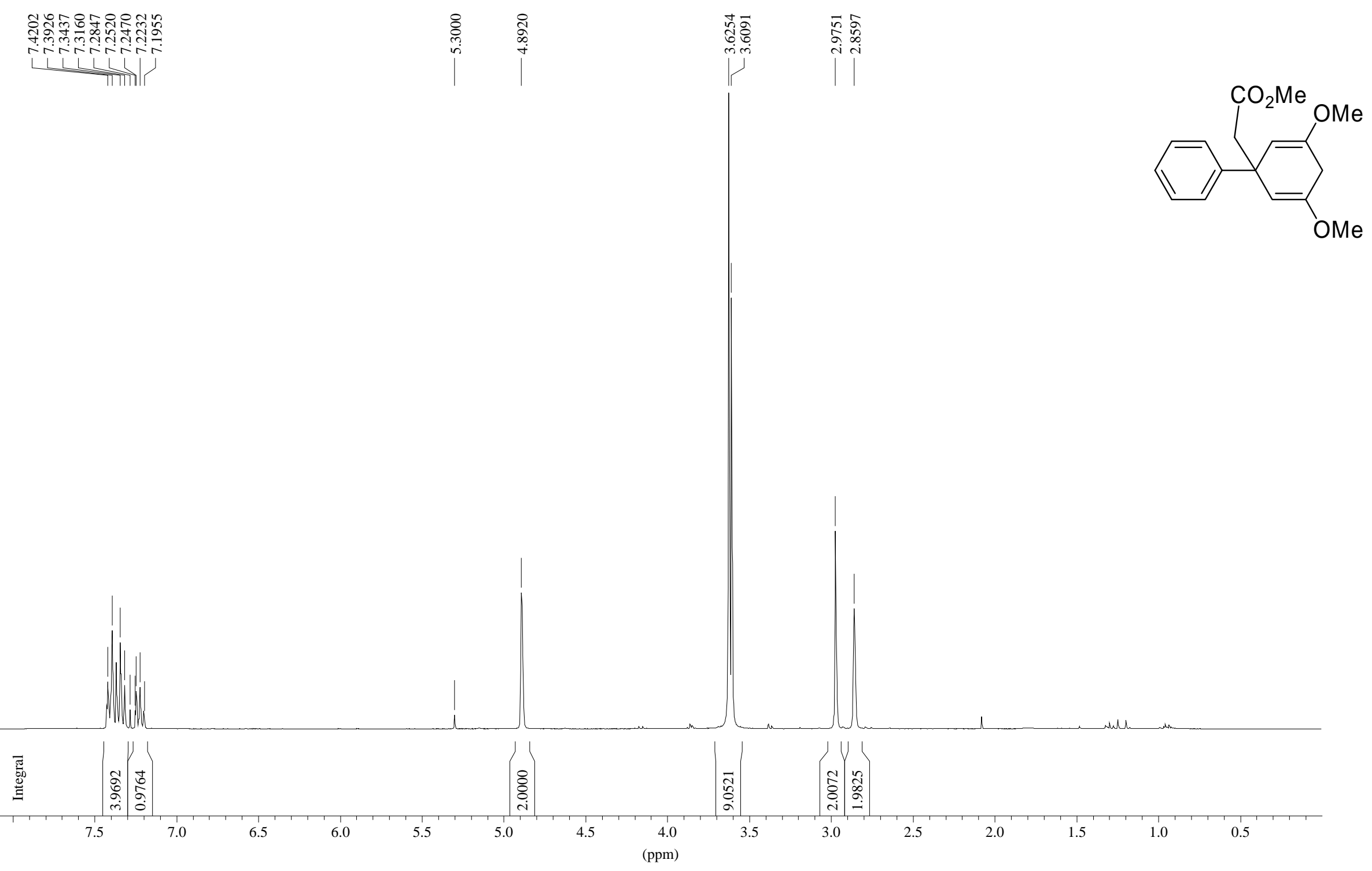




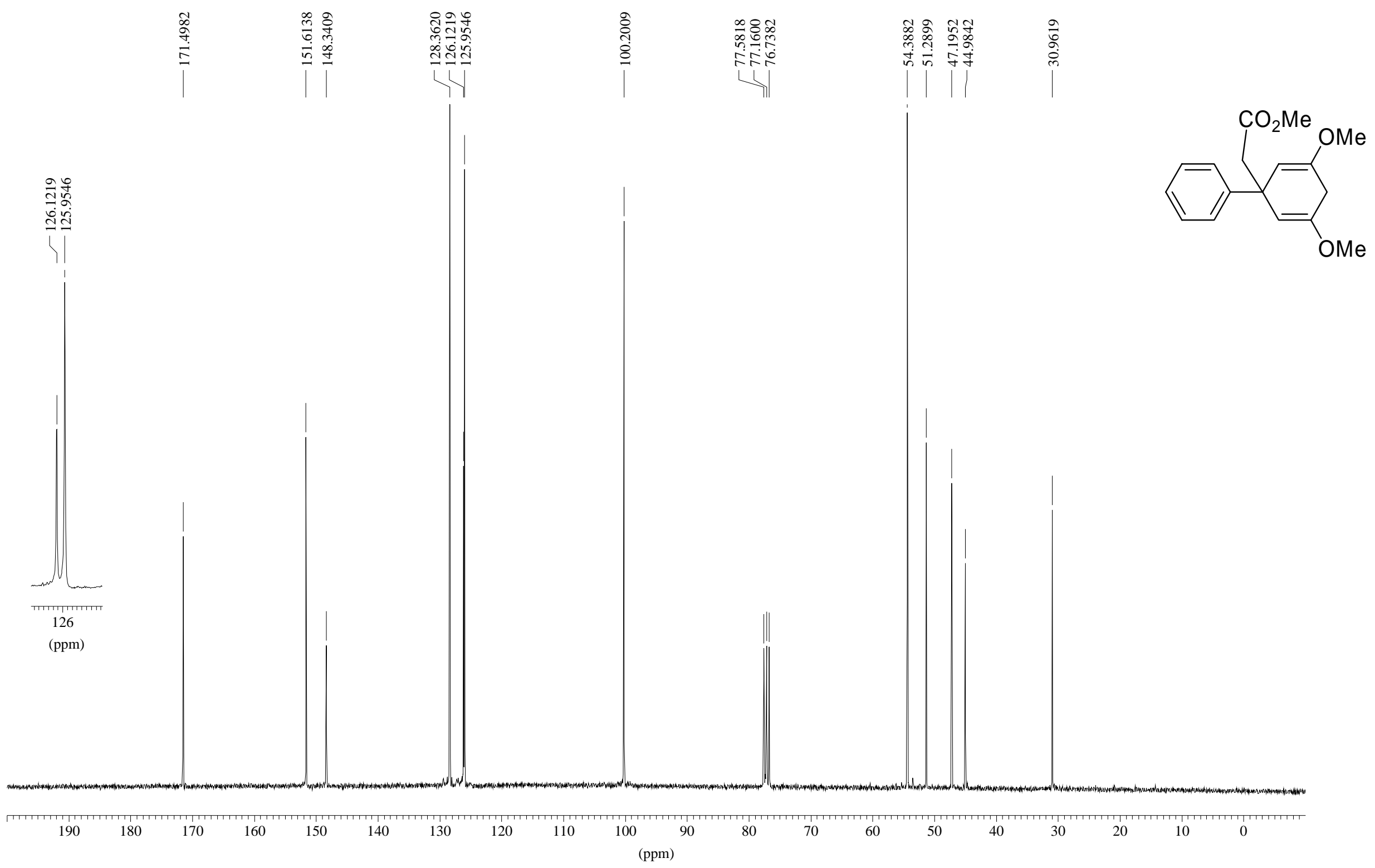




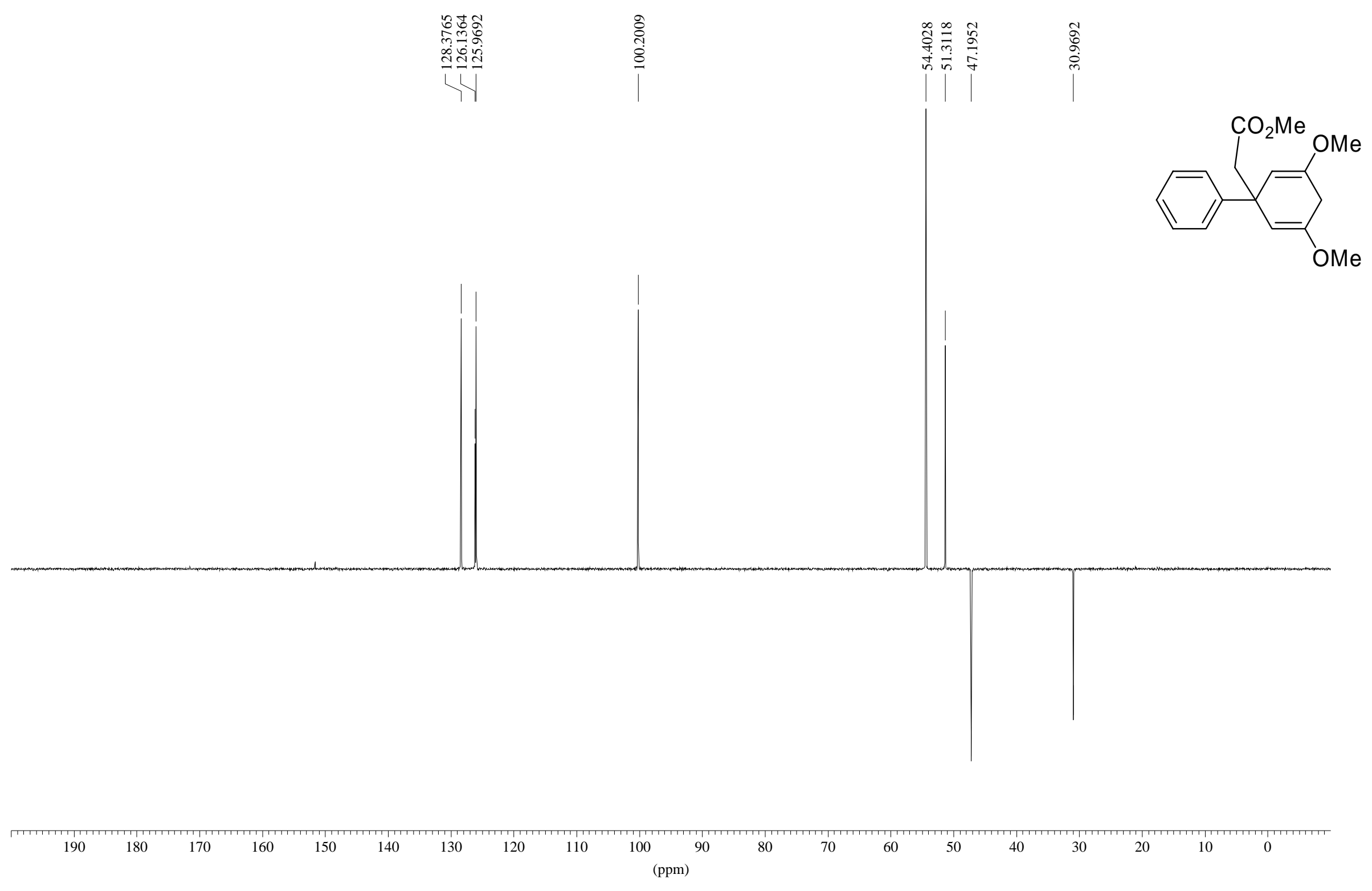




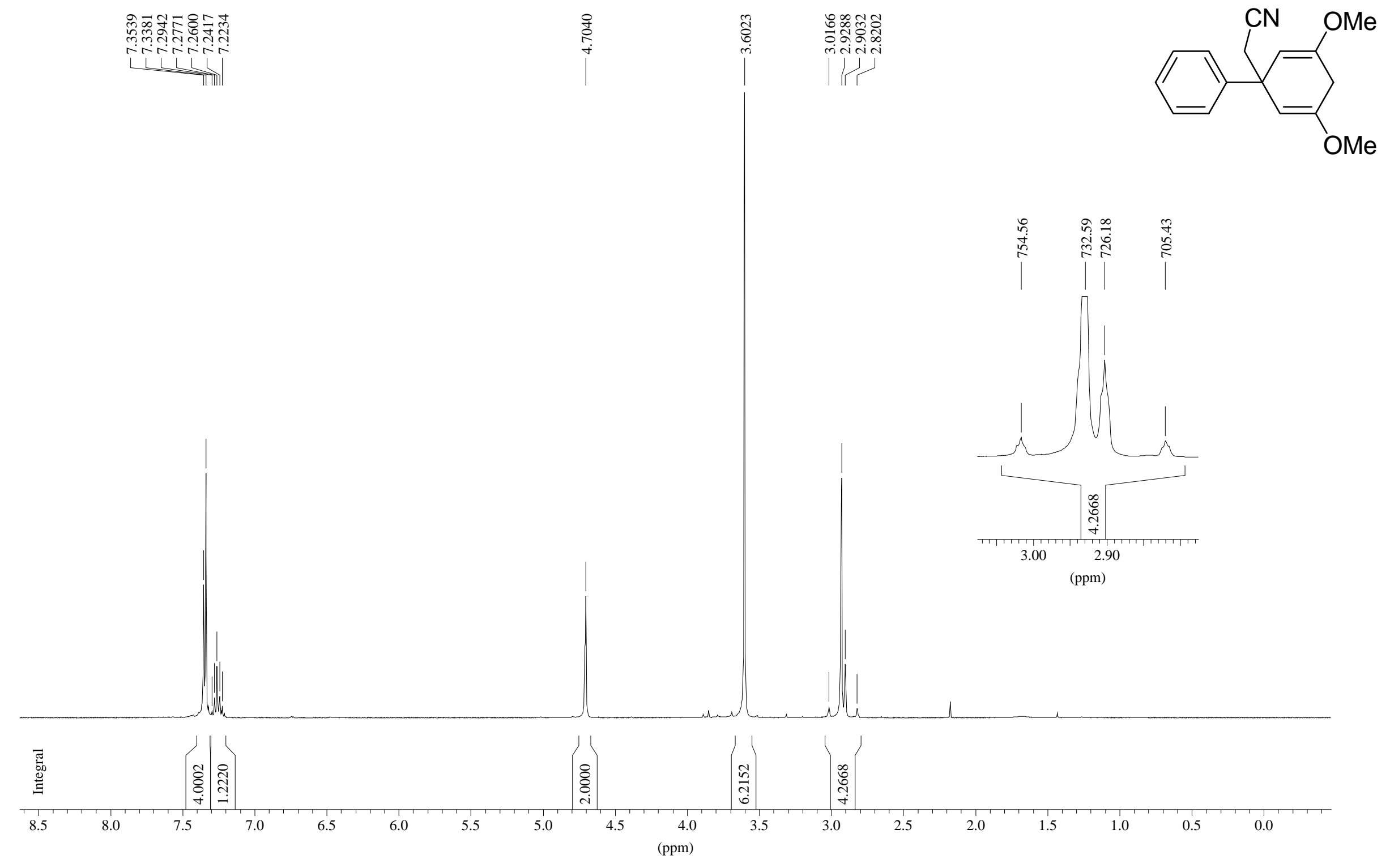




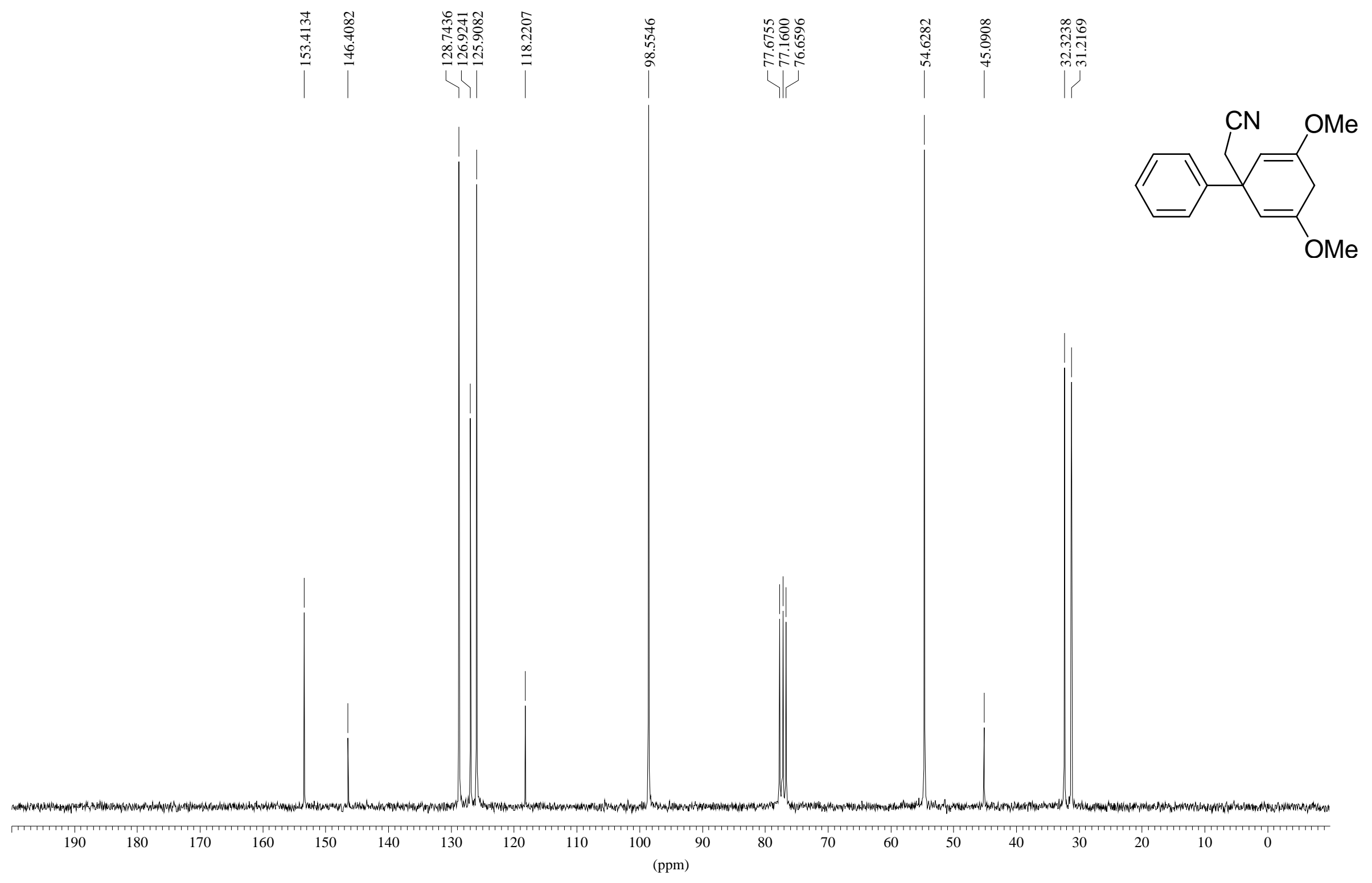




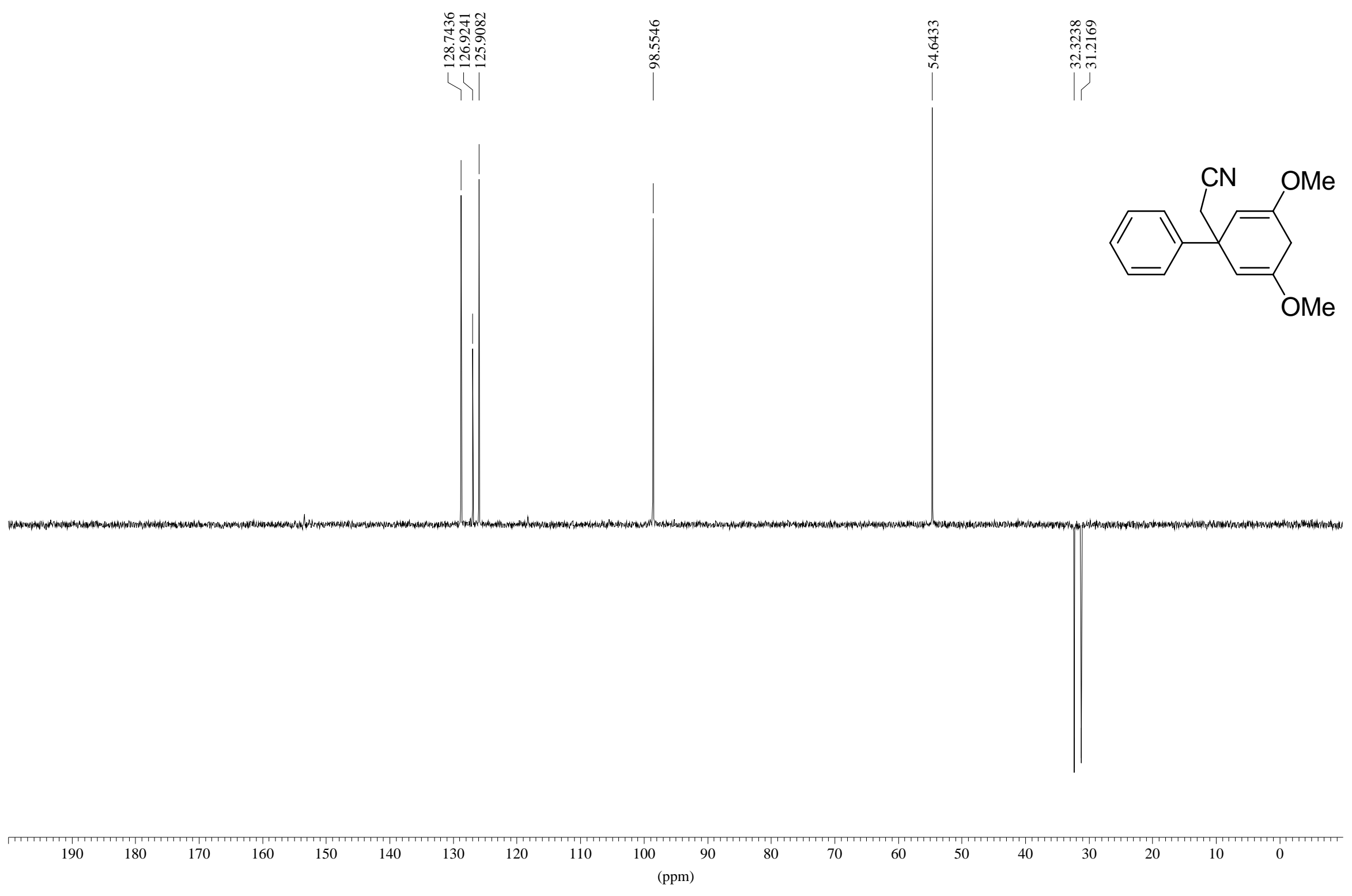




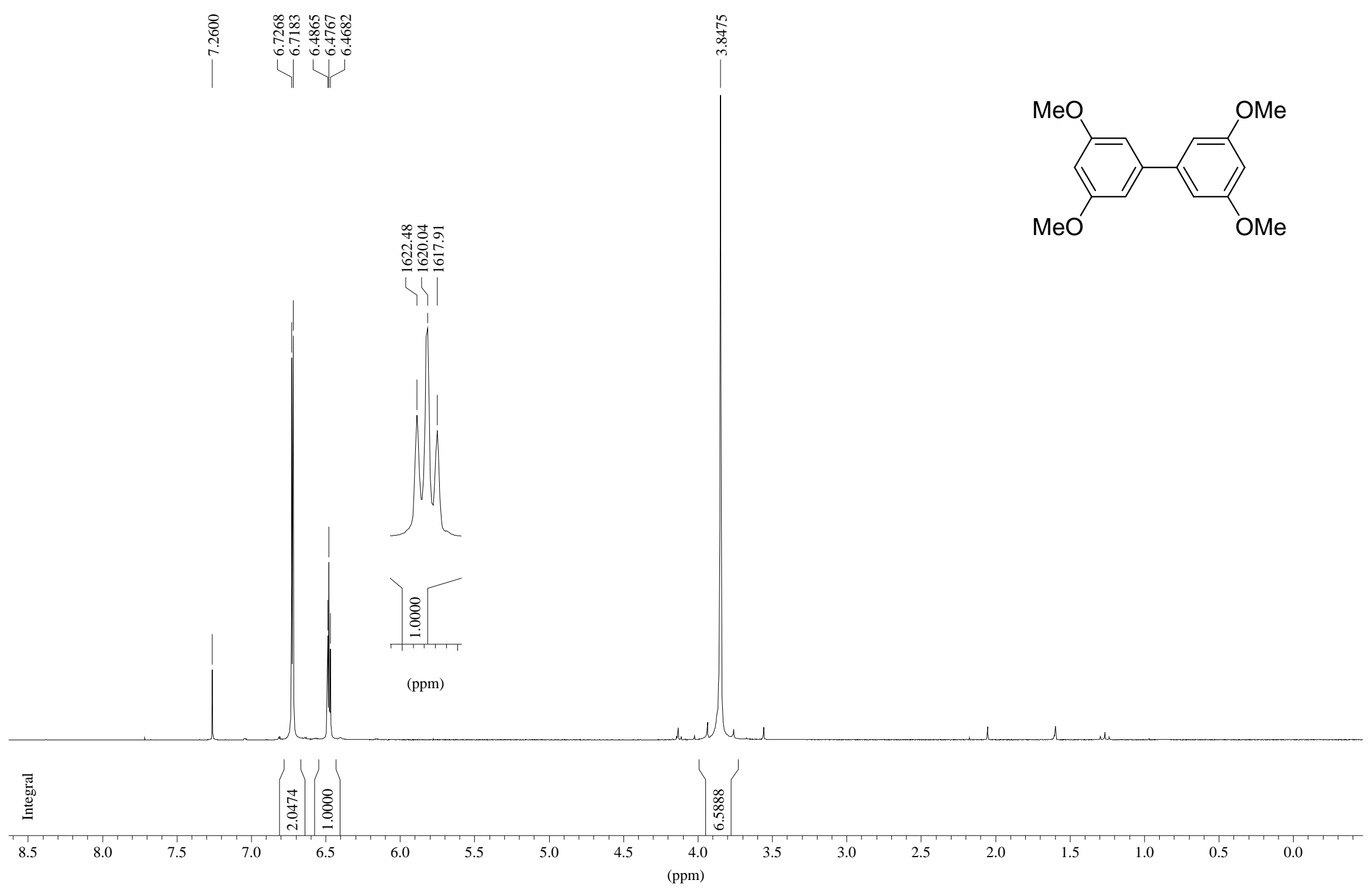




\begin{tabular}{|c|c|c|}
\hline 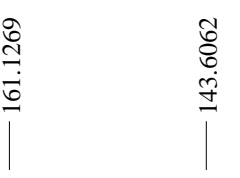 & 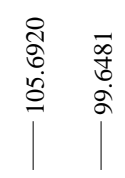 & 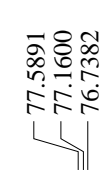 \\
\hline
\end{tabular}
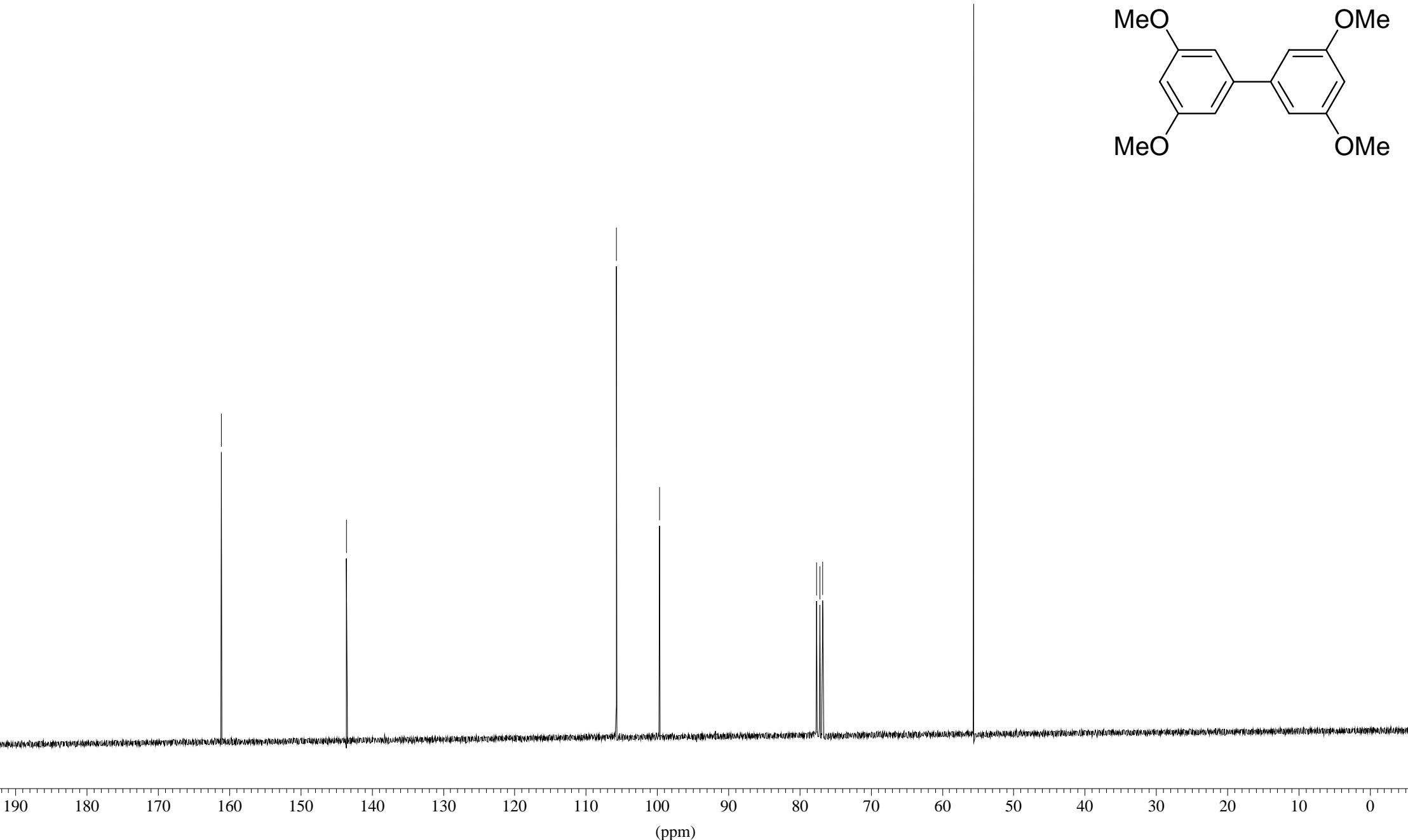


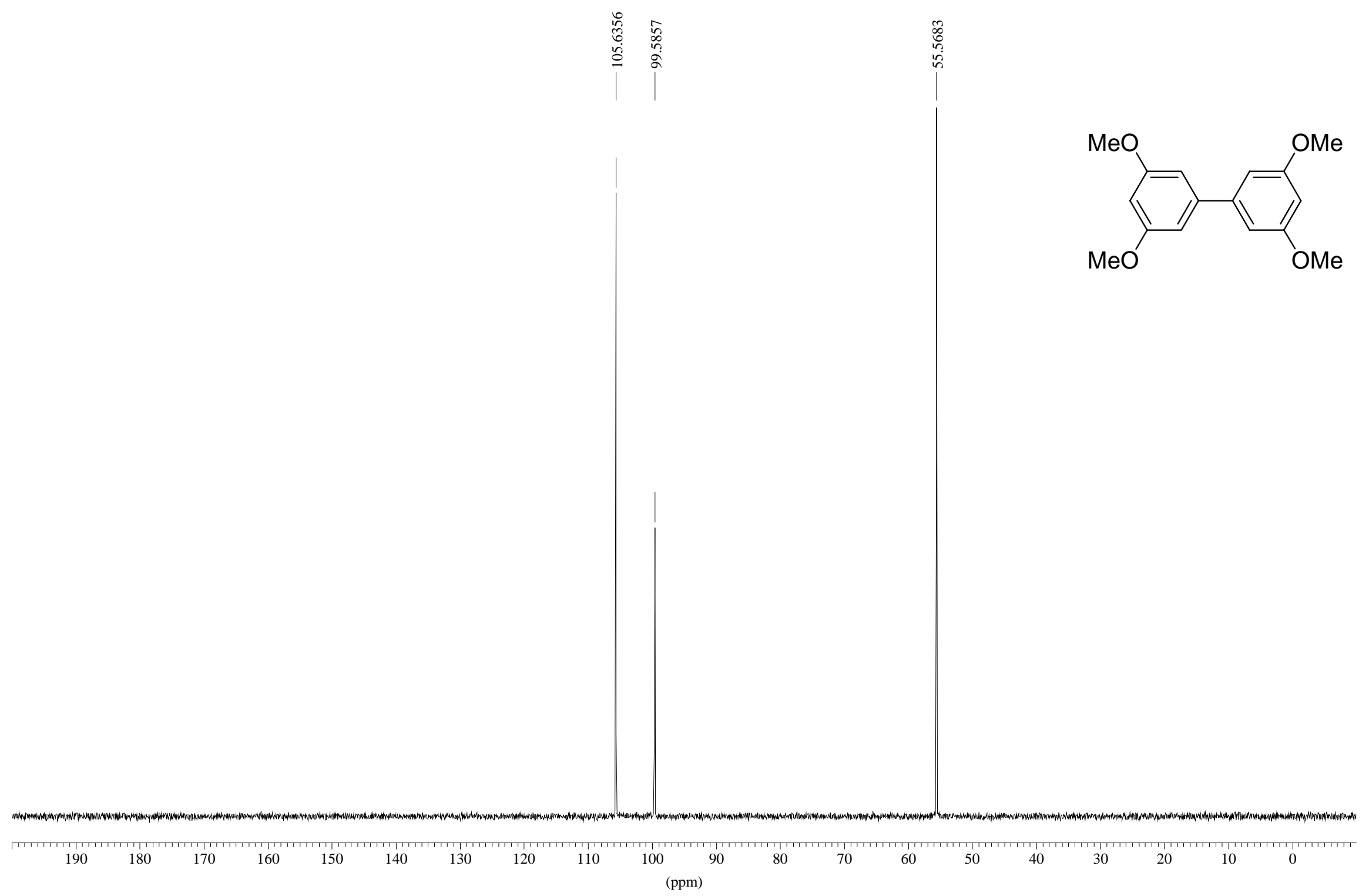




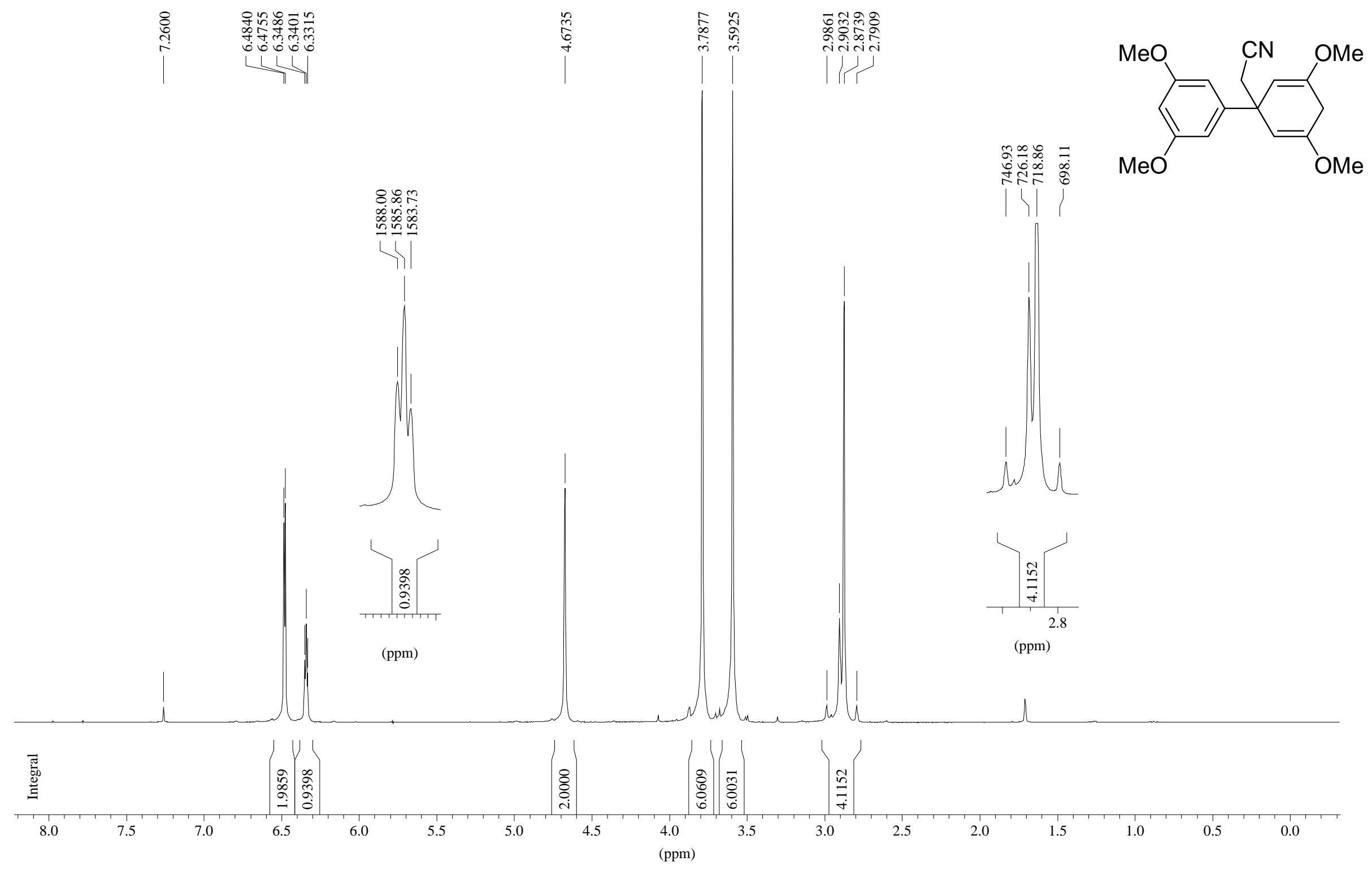




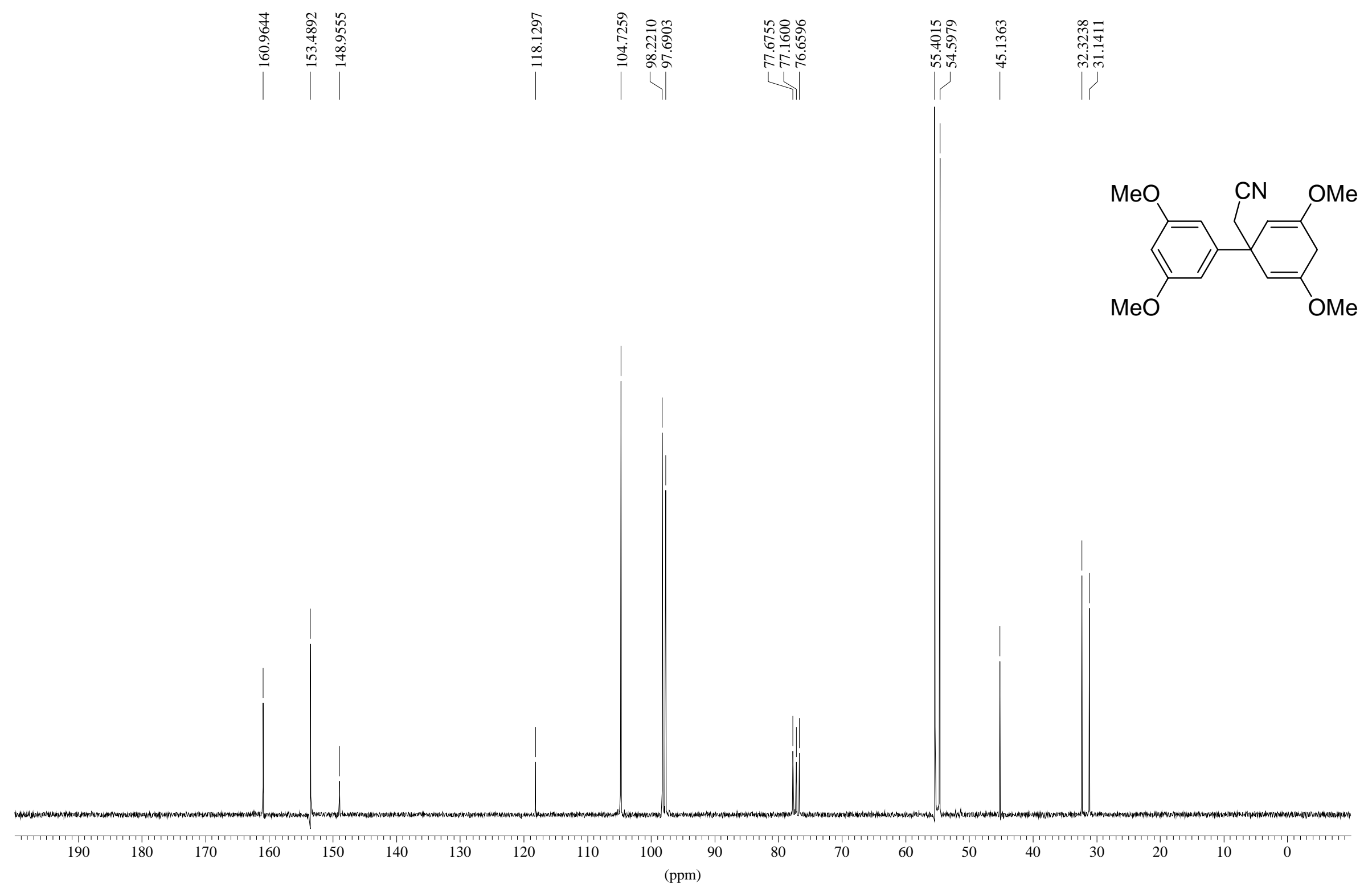




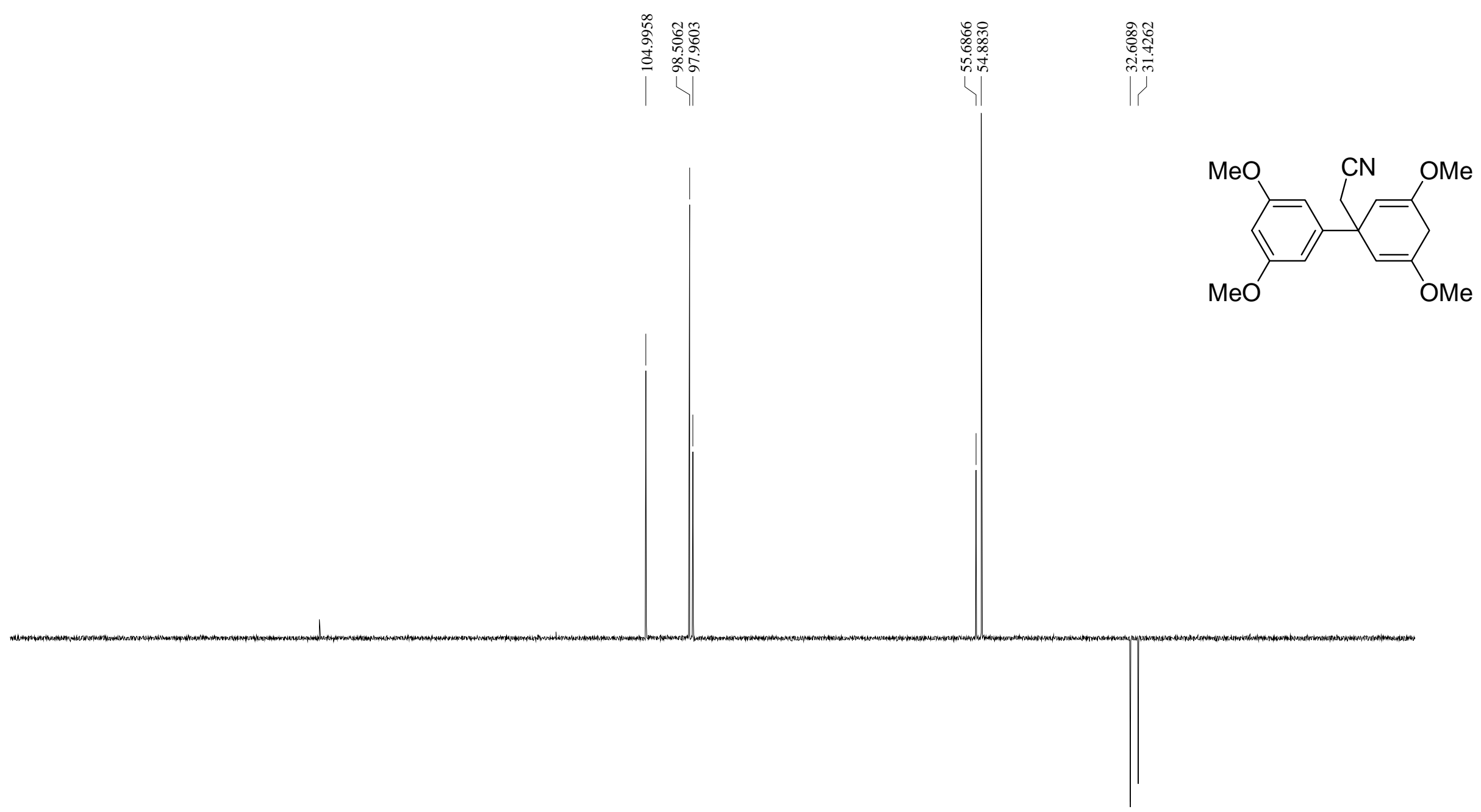

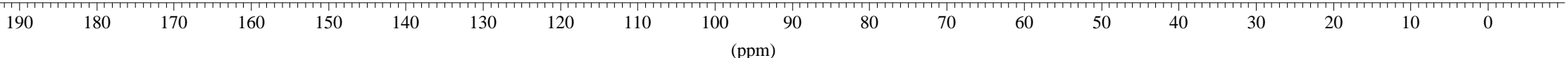




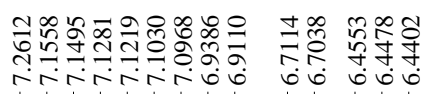

곡웡율

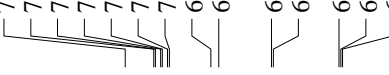

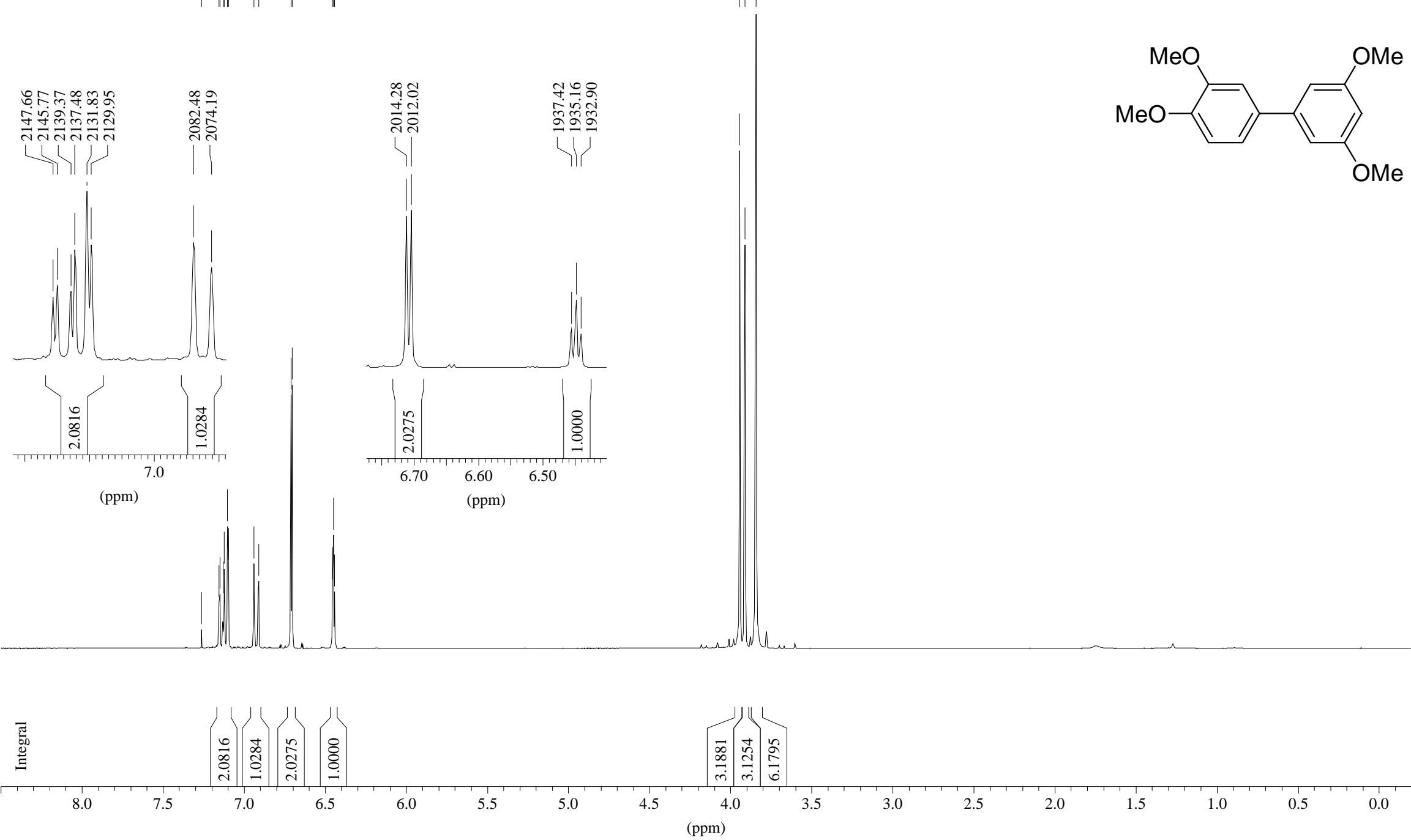




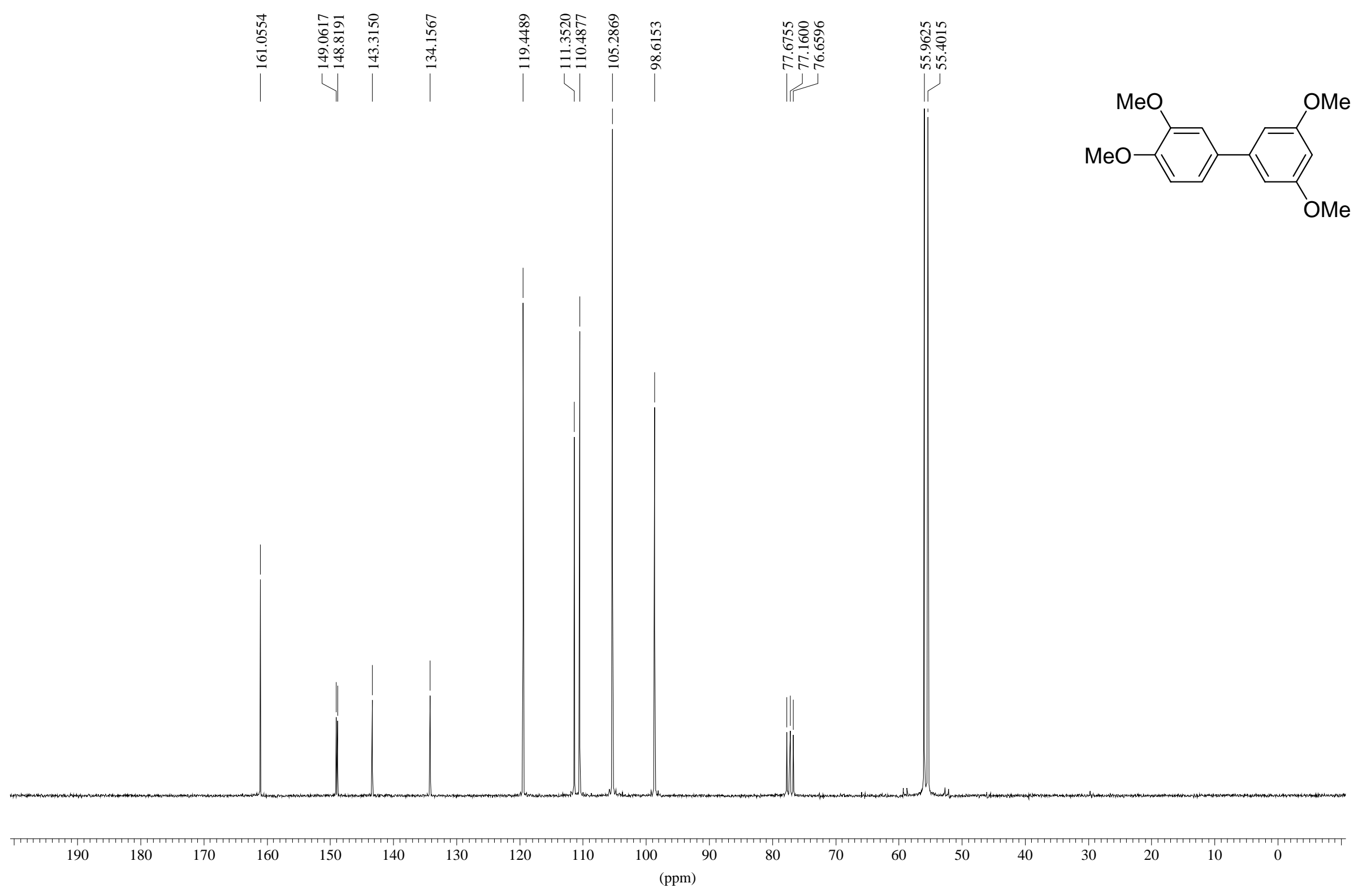




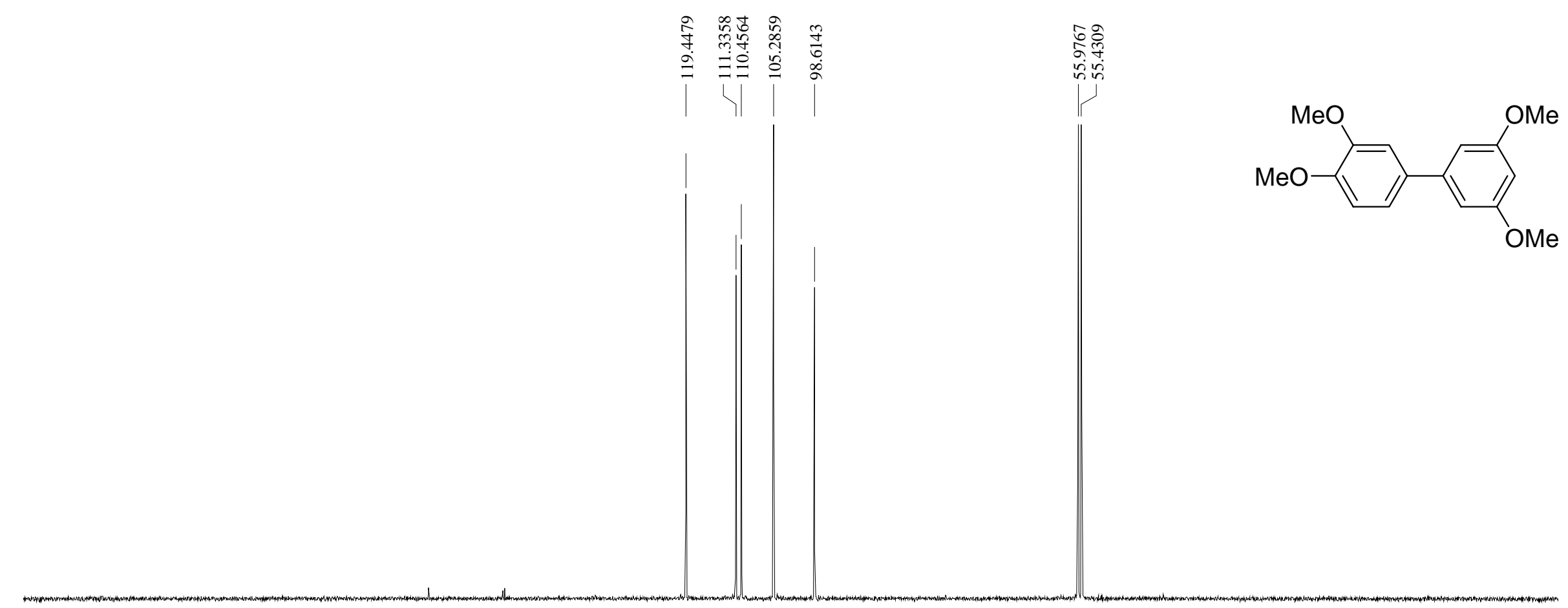




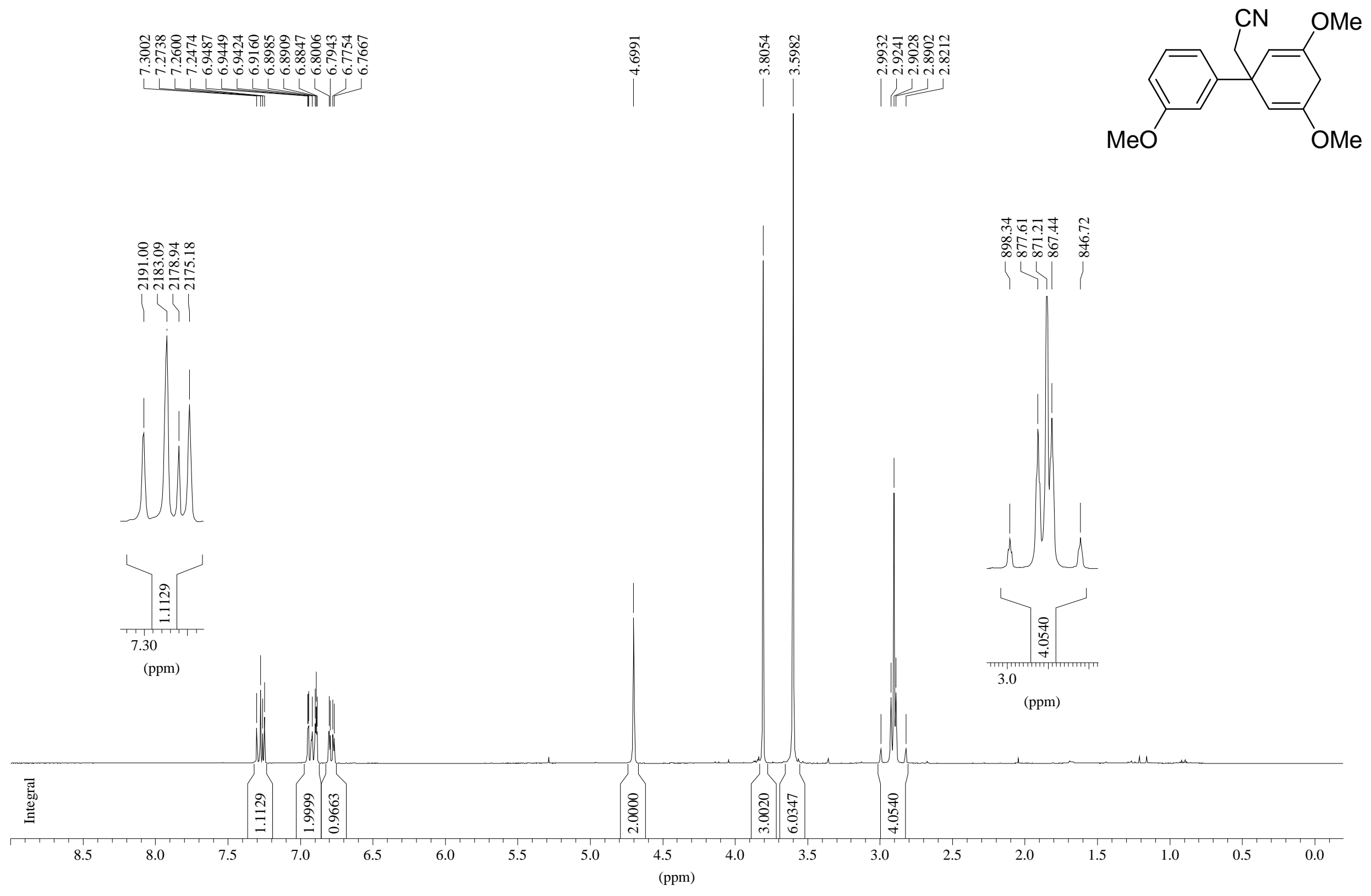




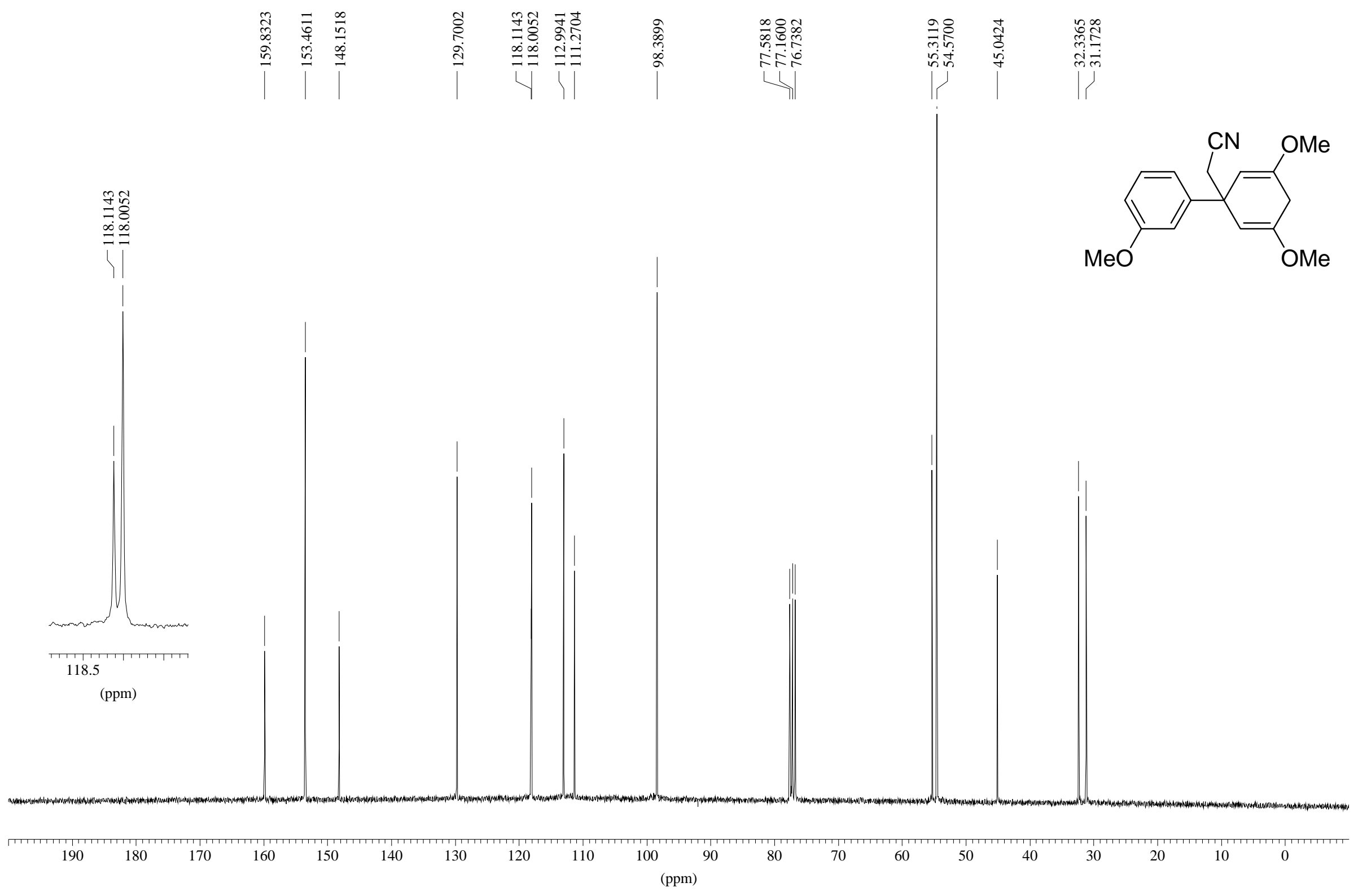




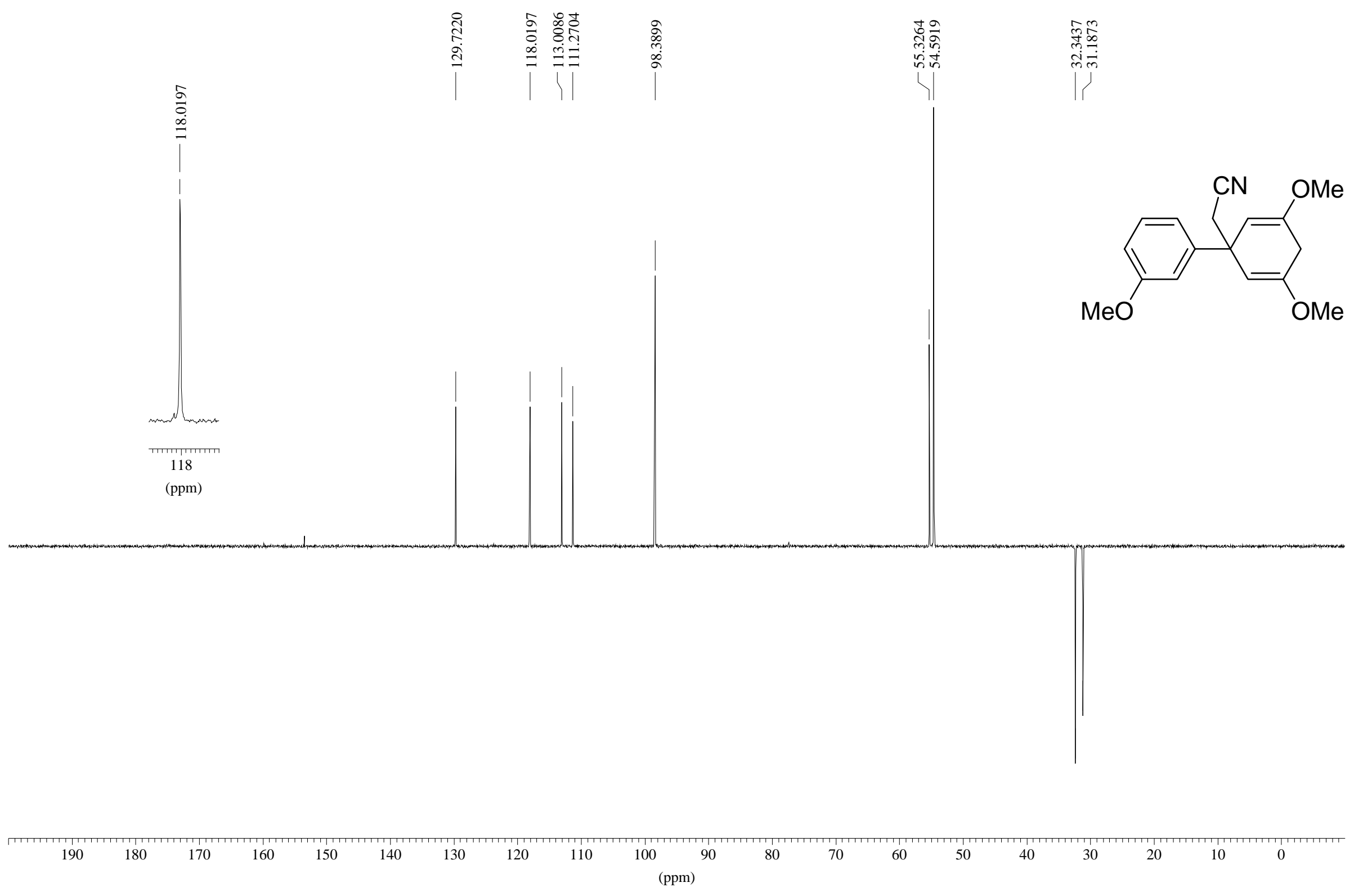




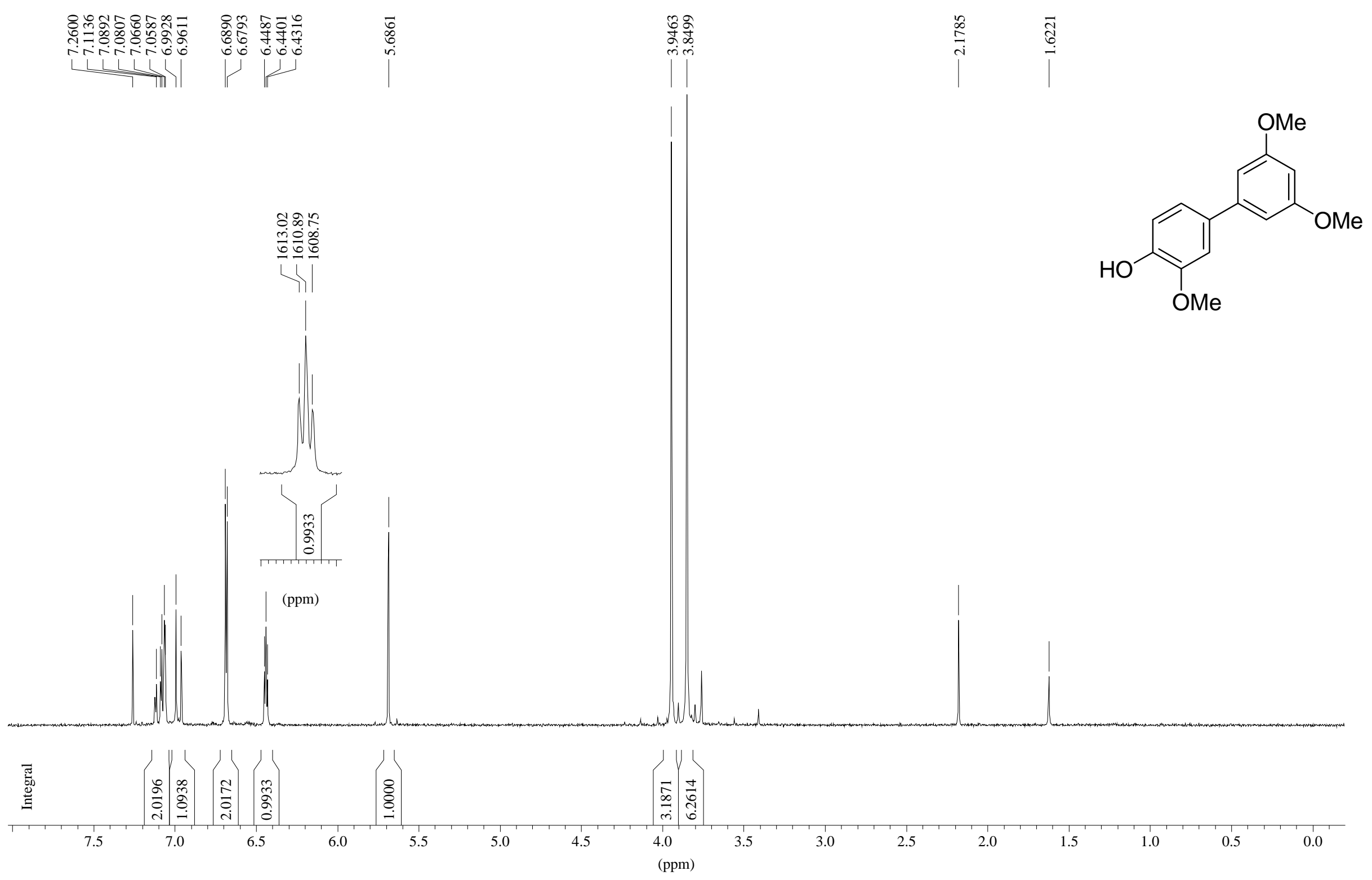




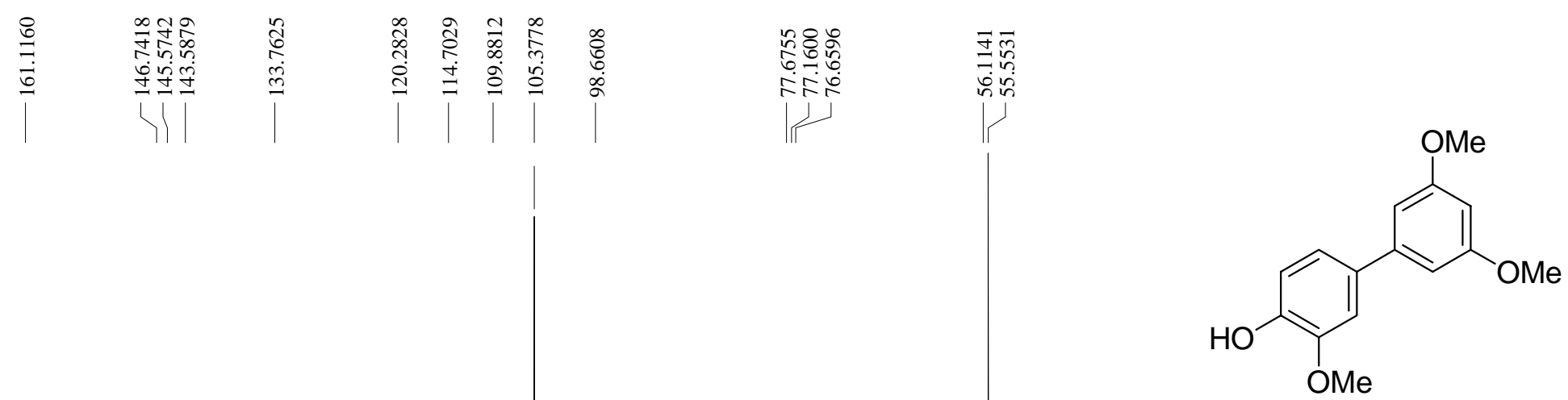




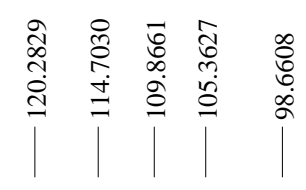

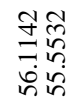

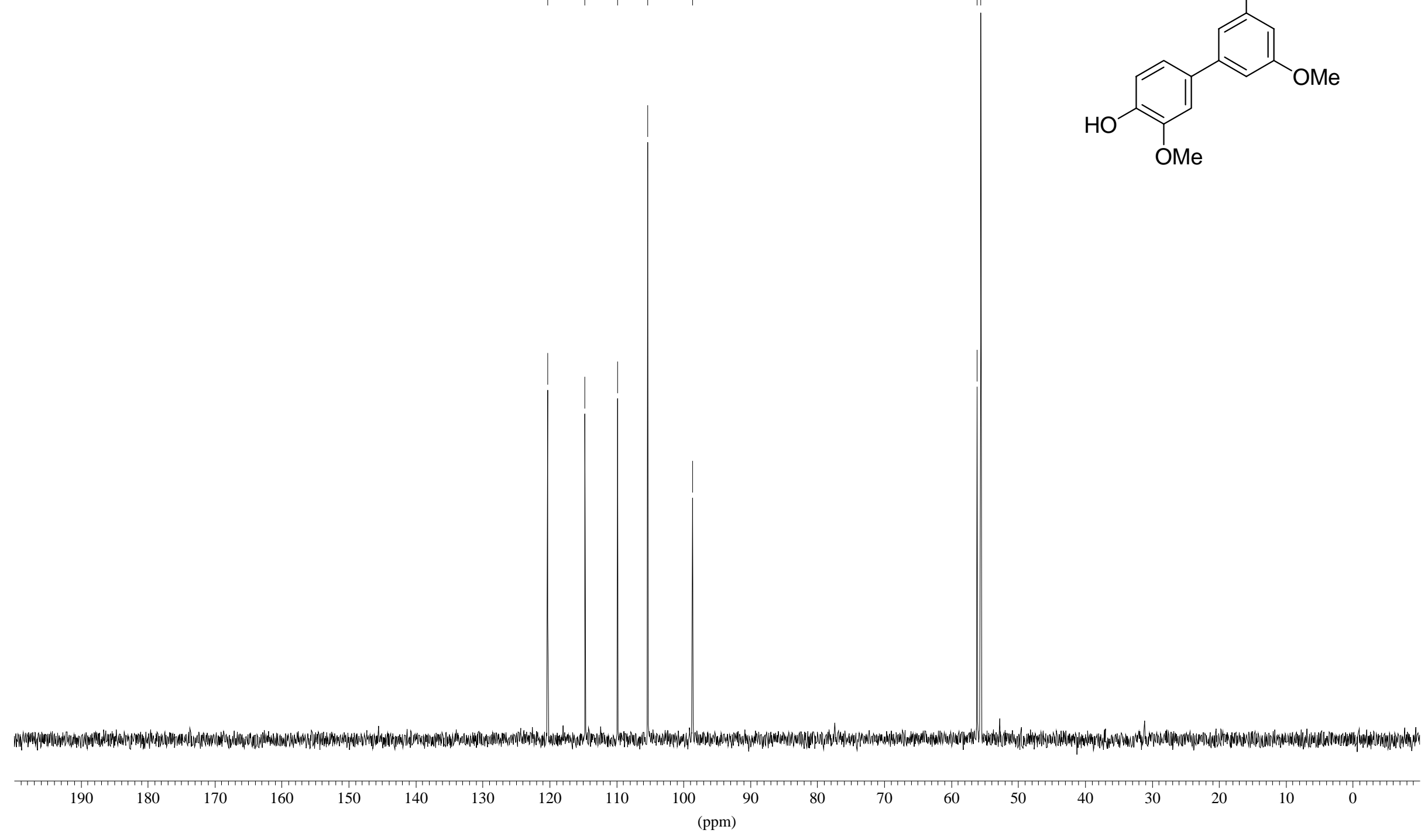




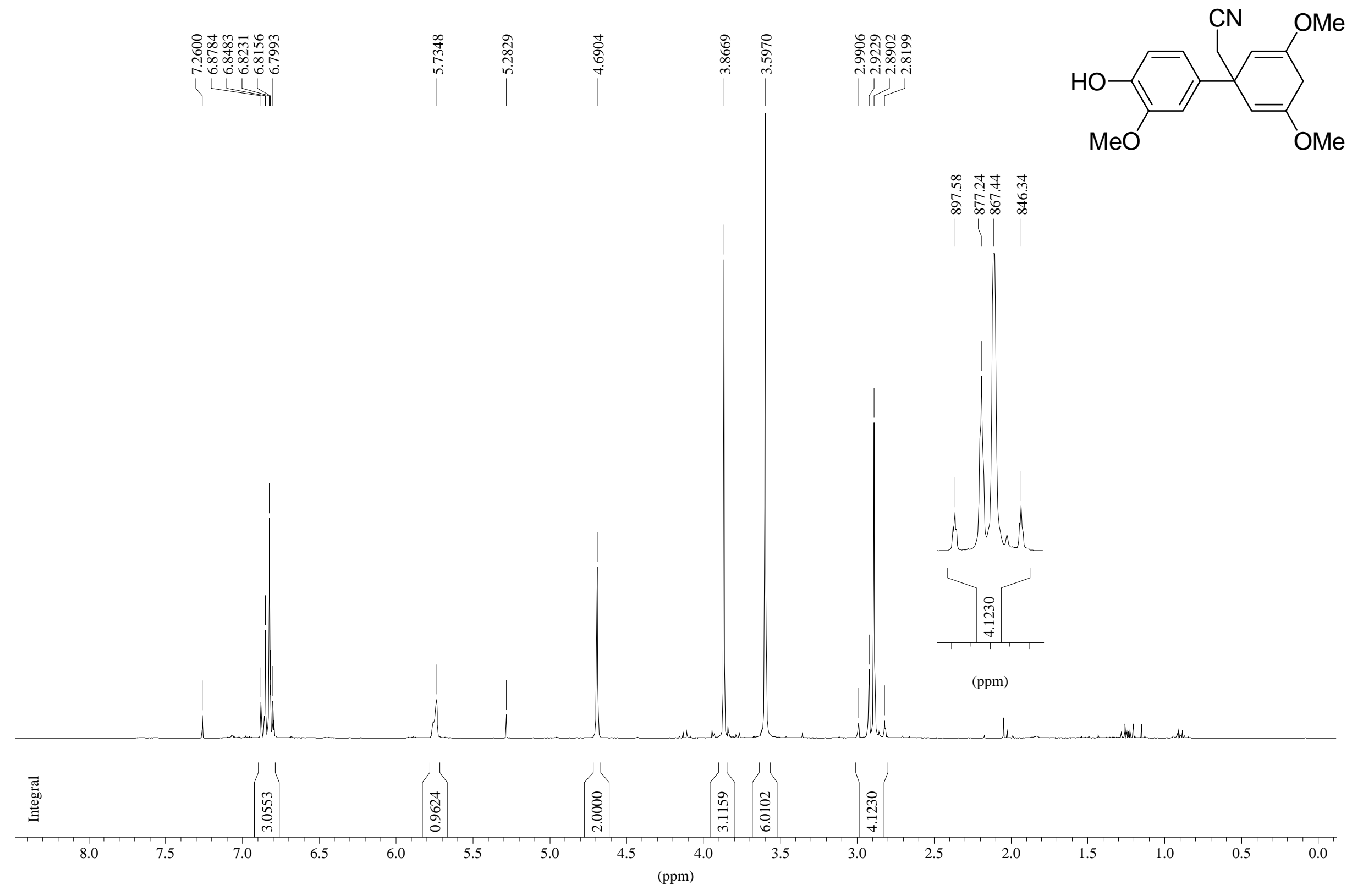




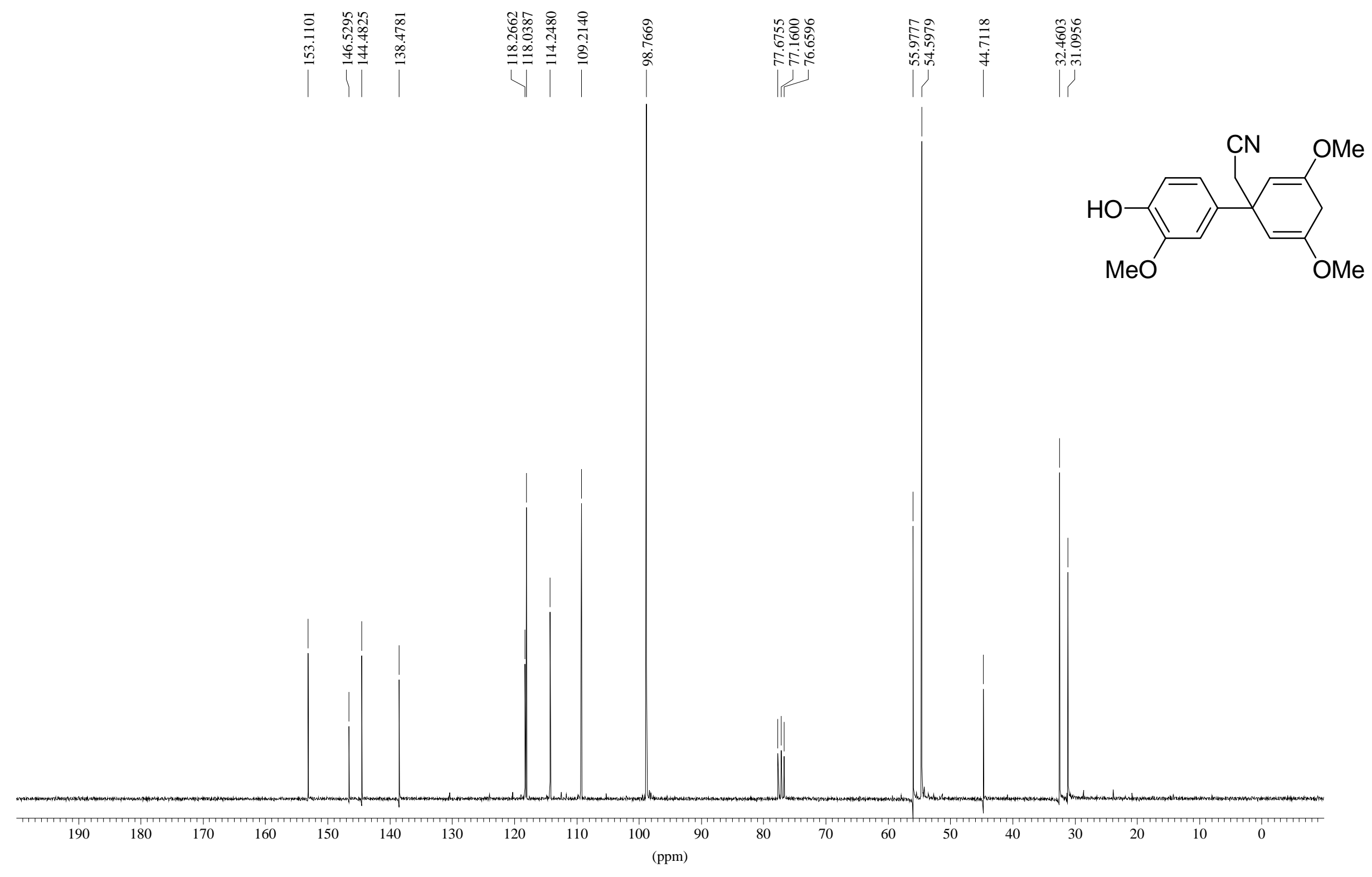




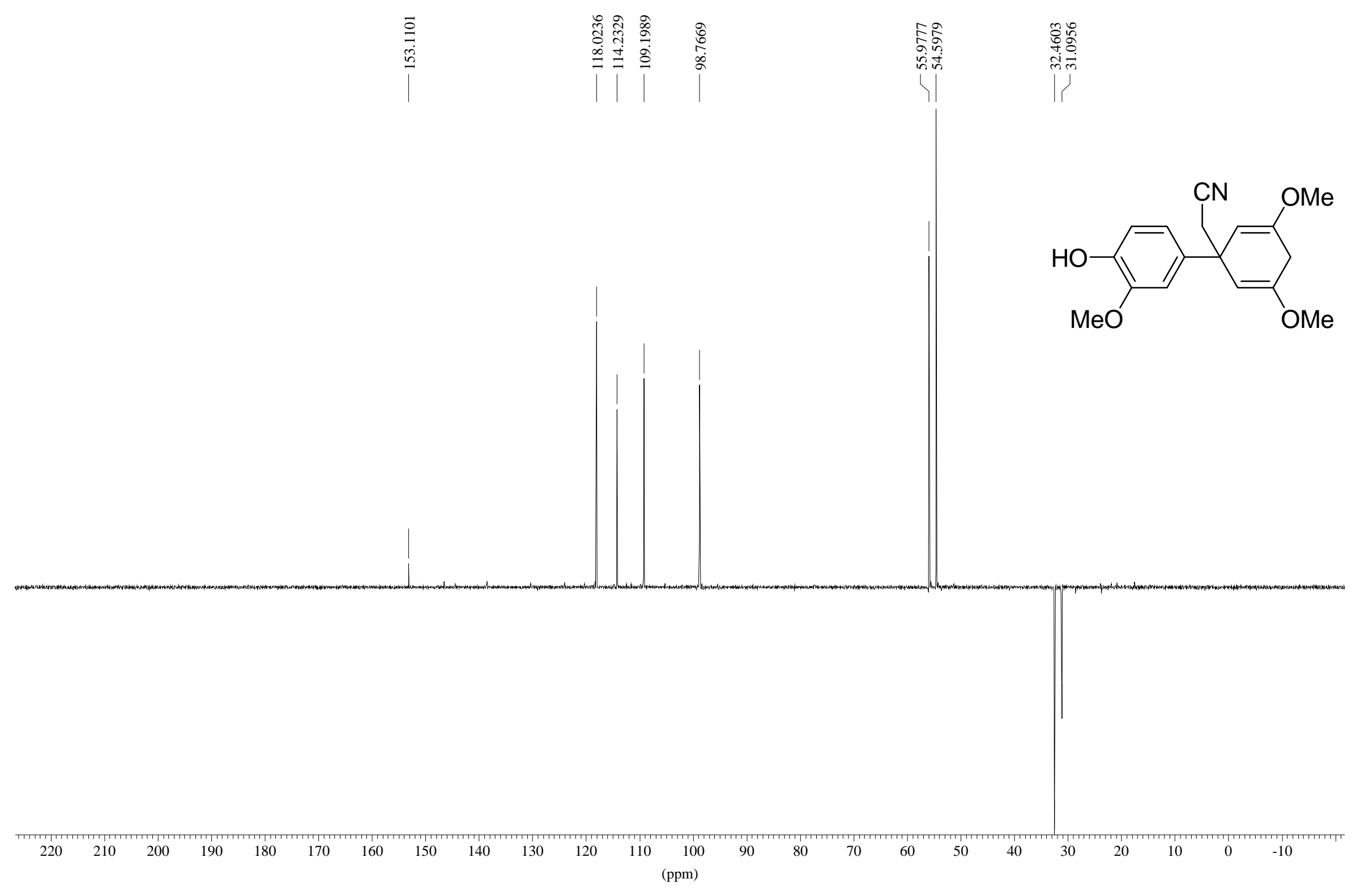




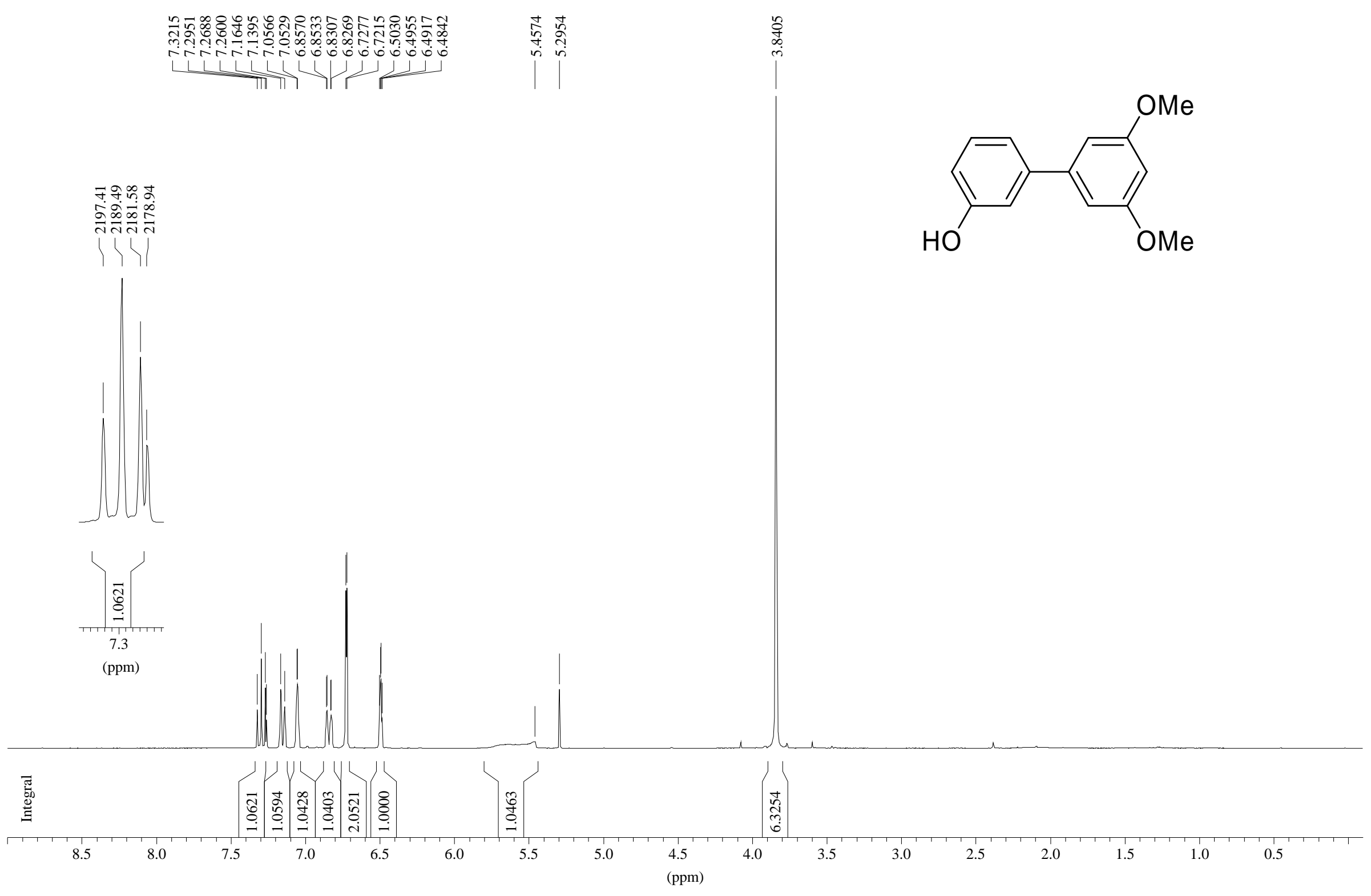




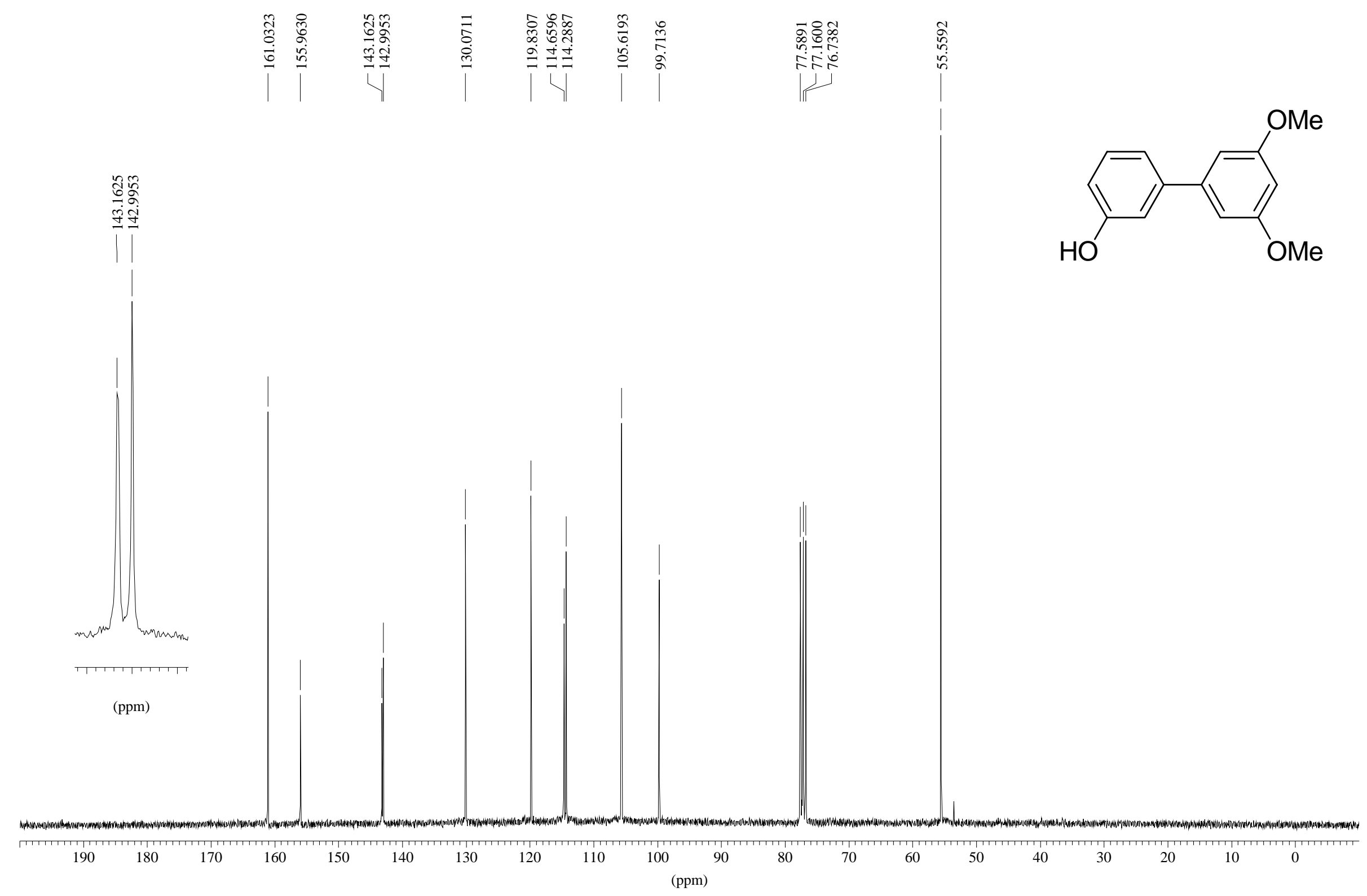




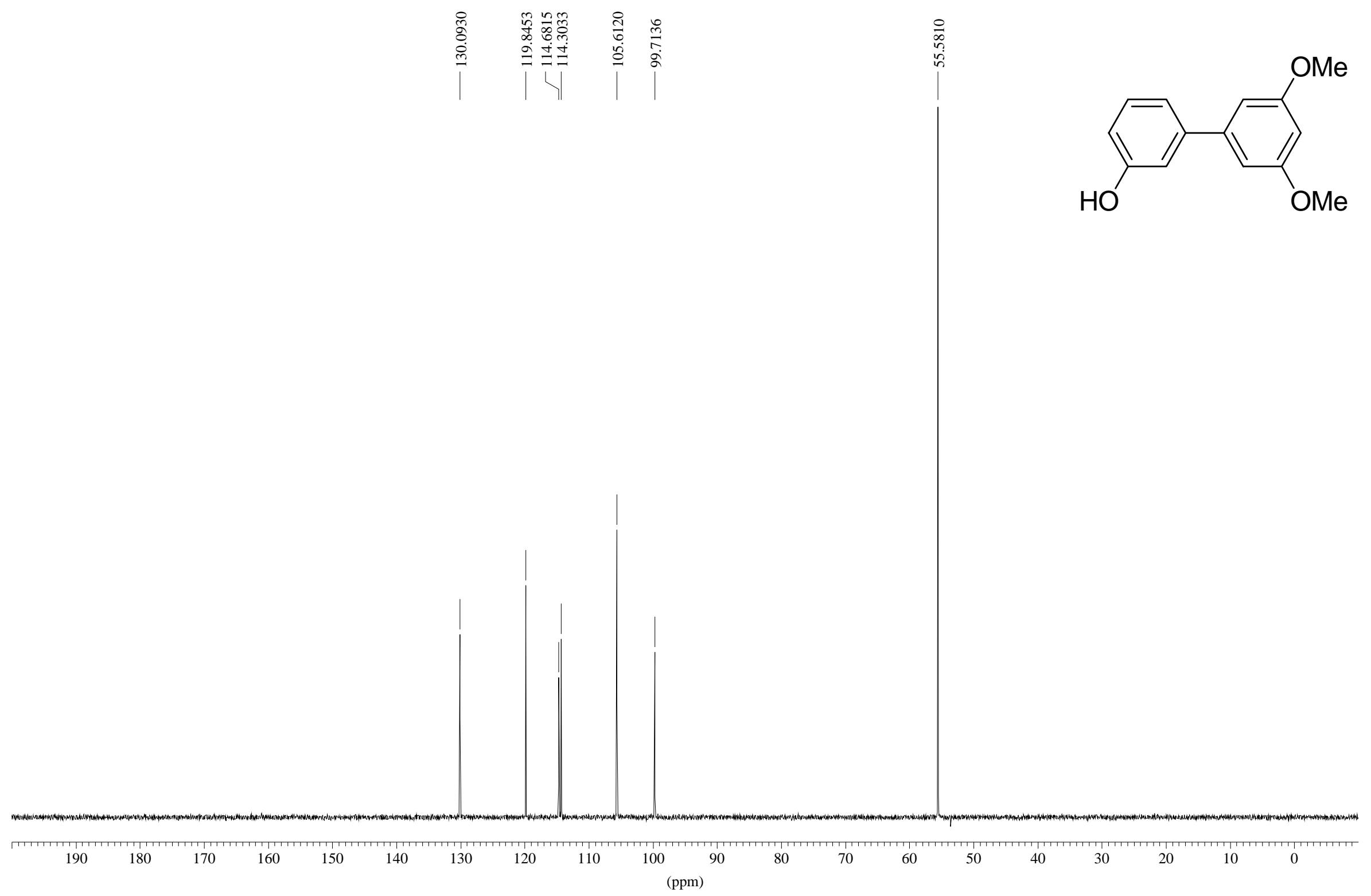




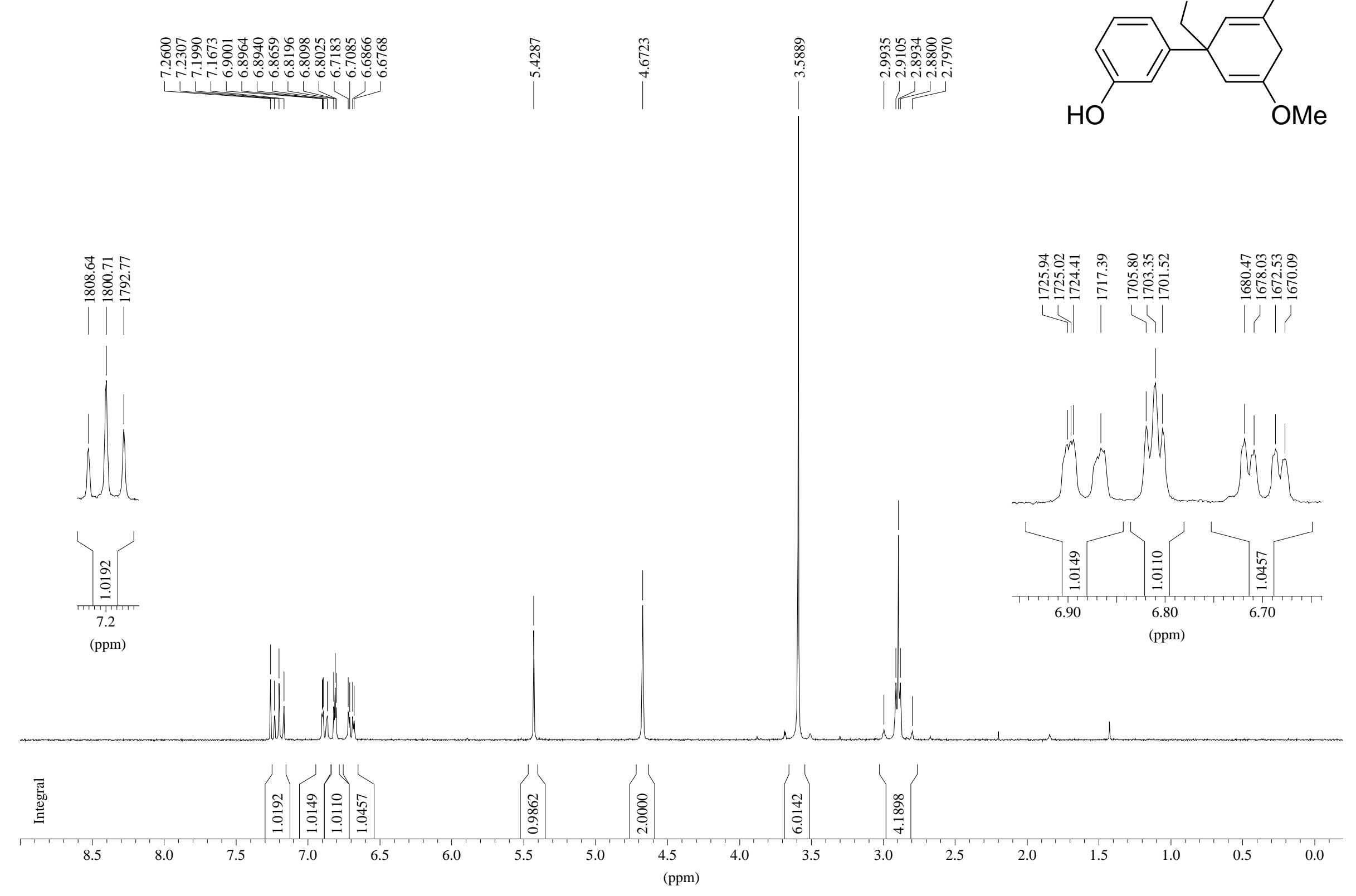




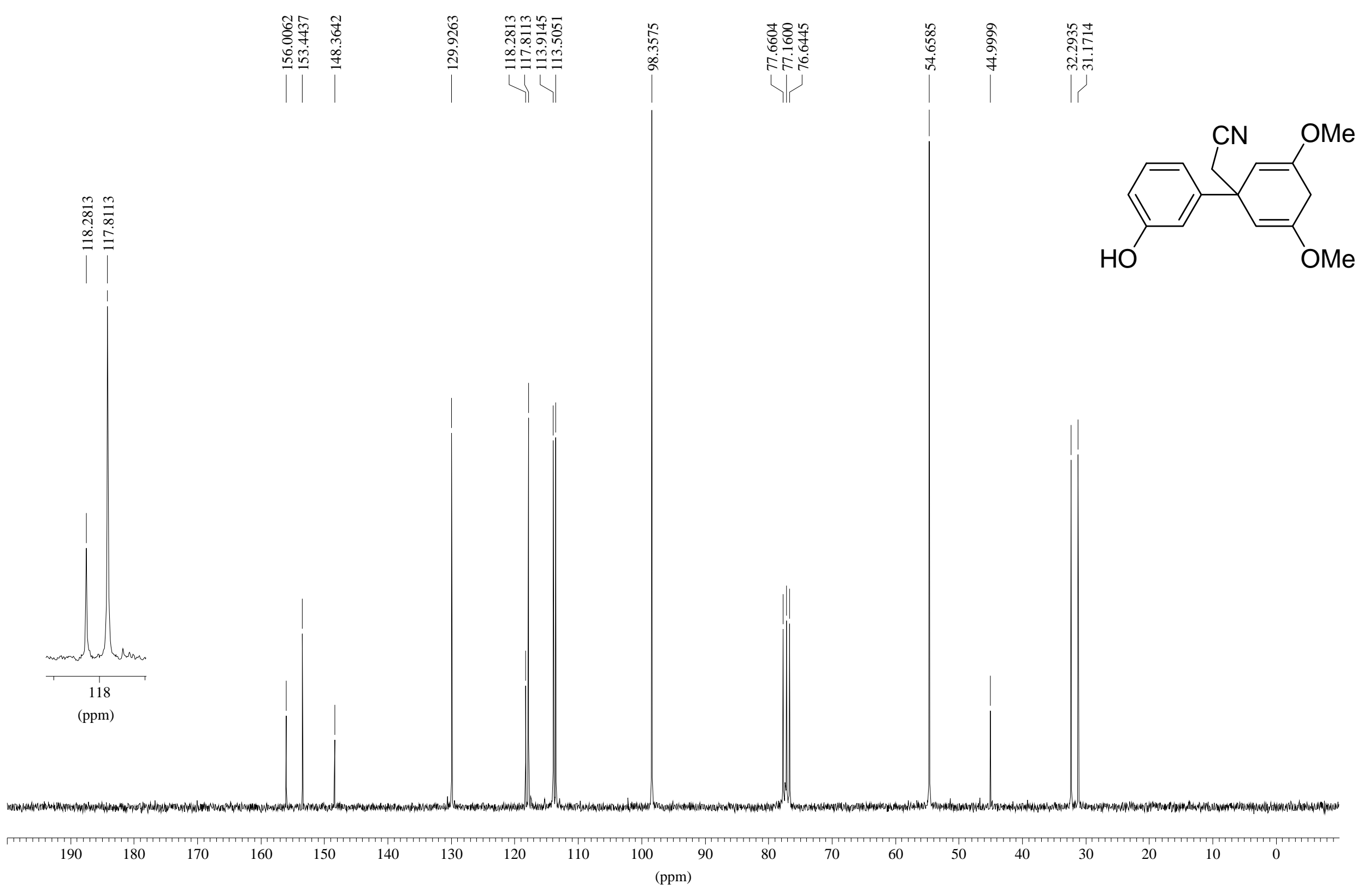




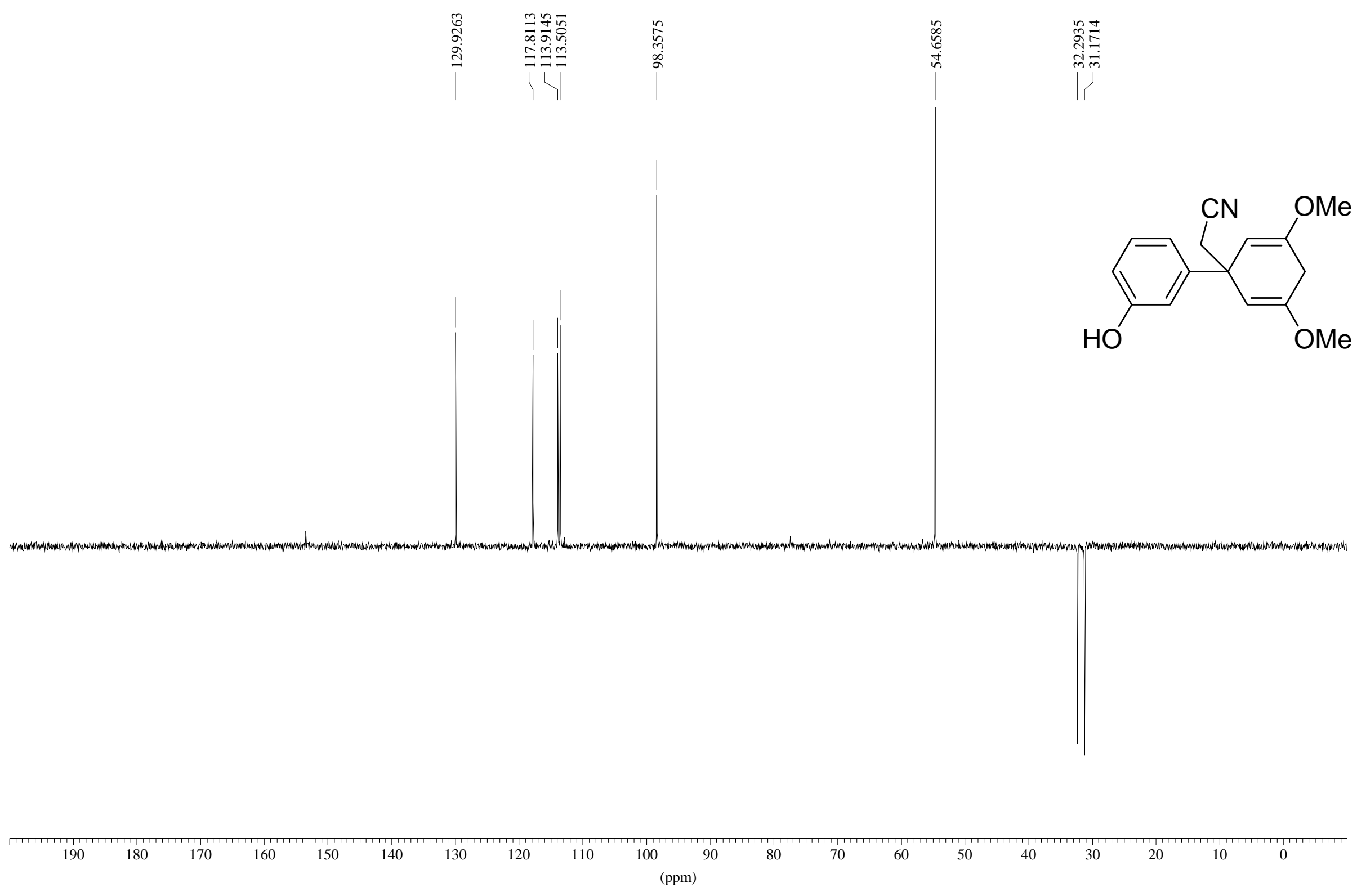




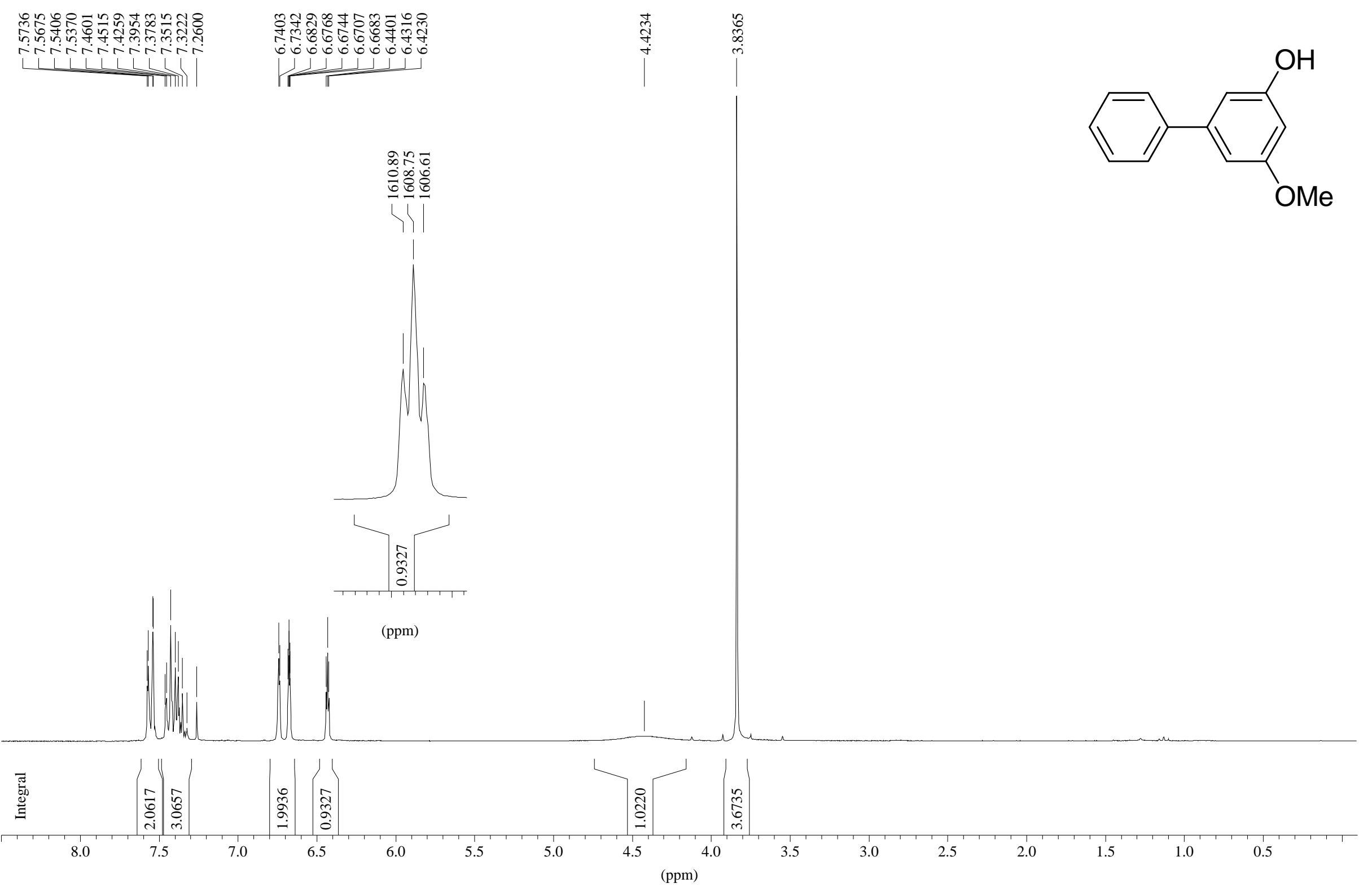




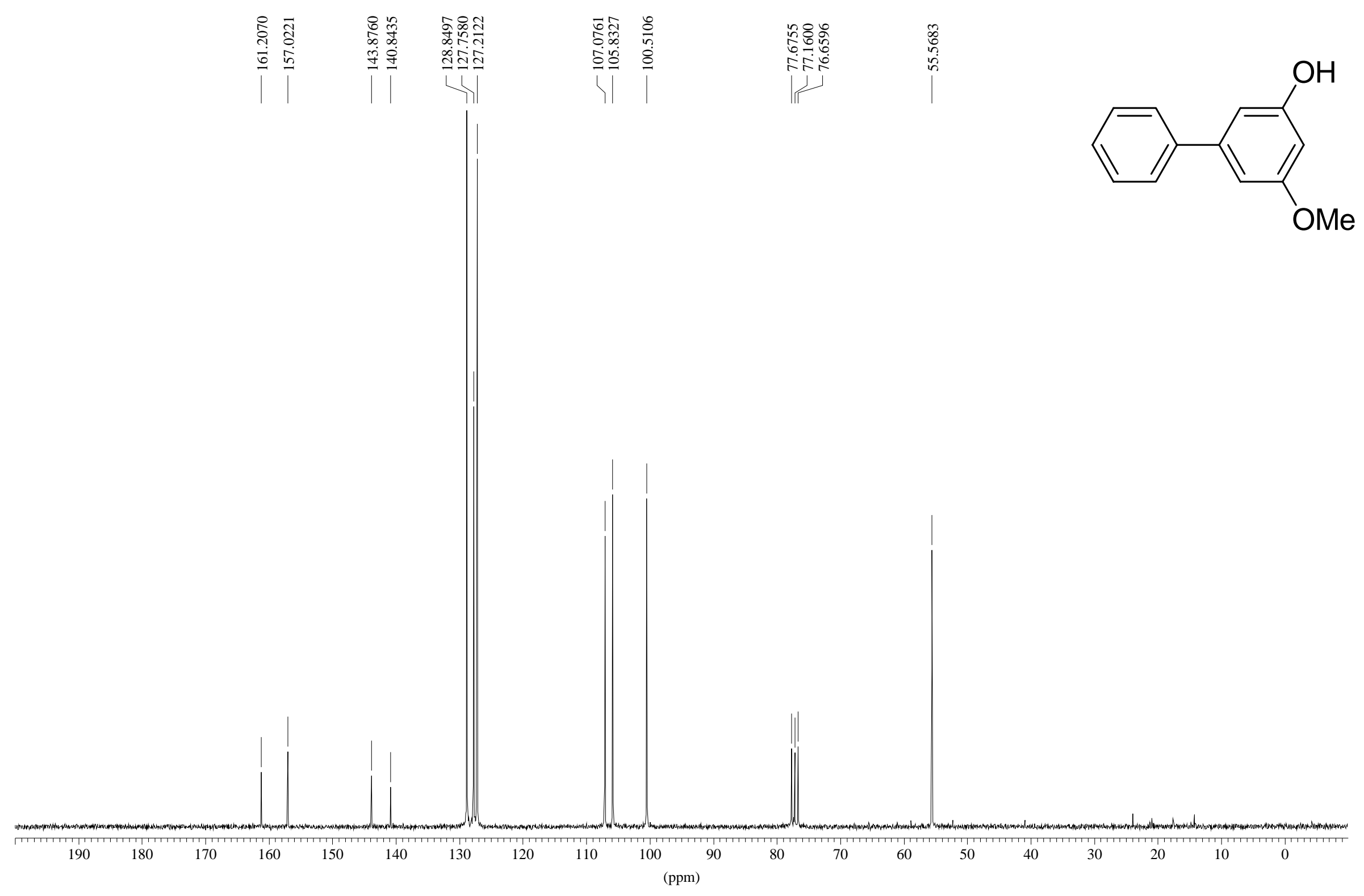




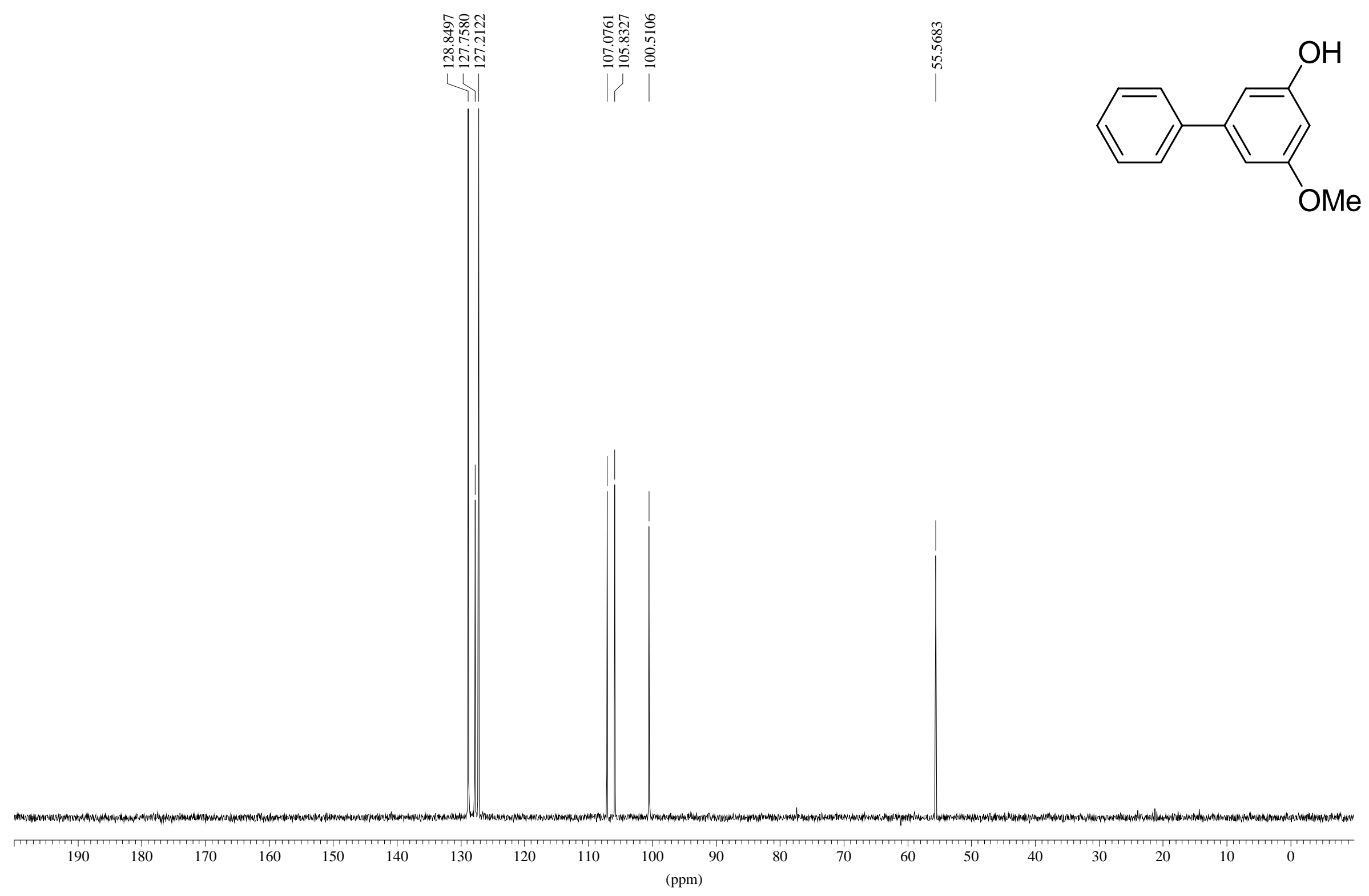




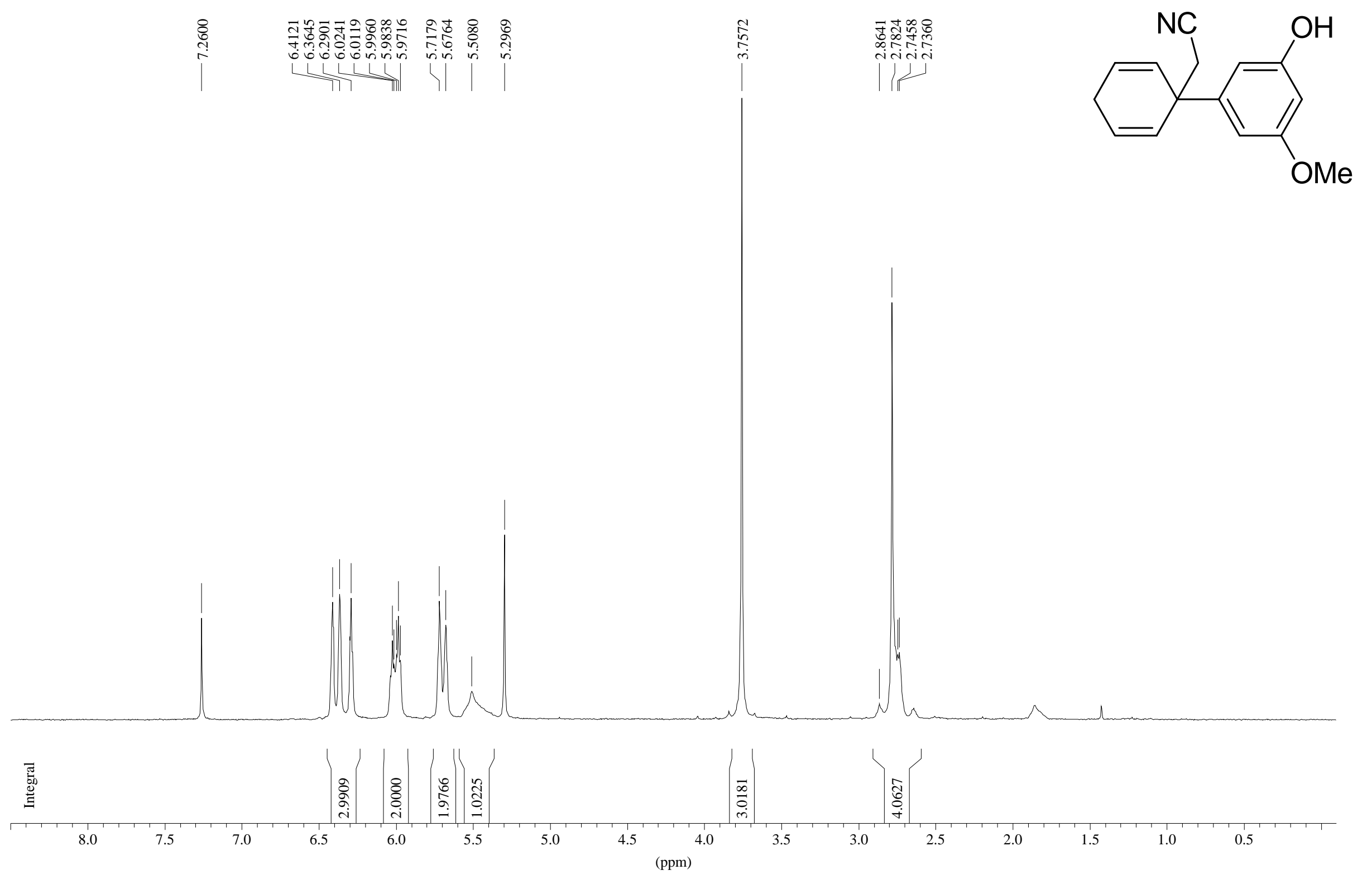



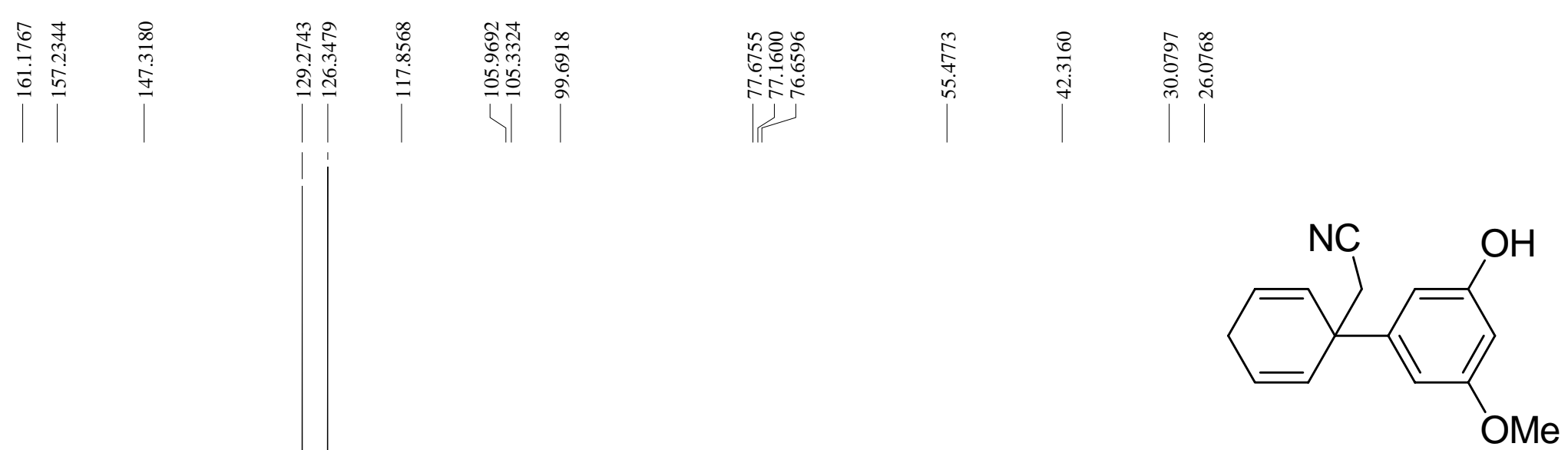

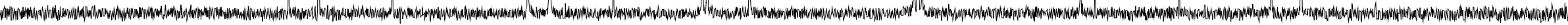

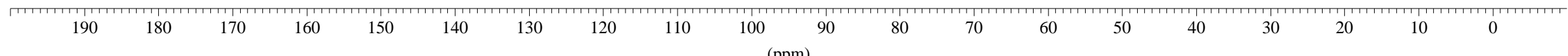




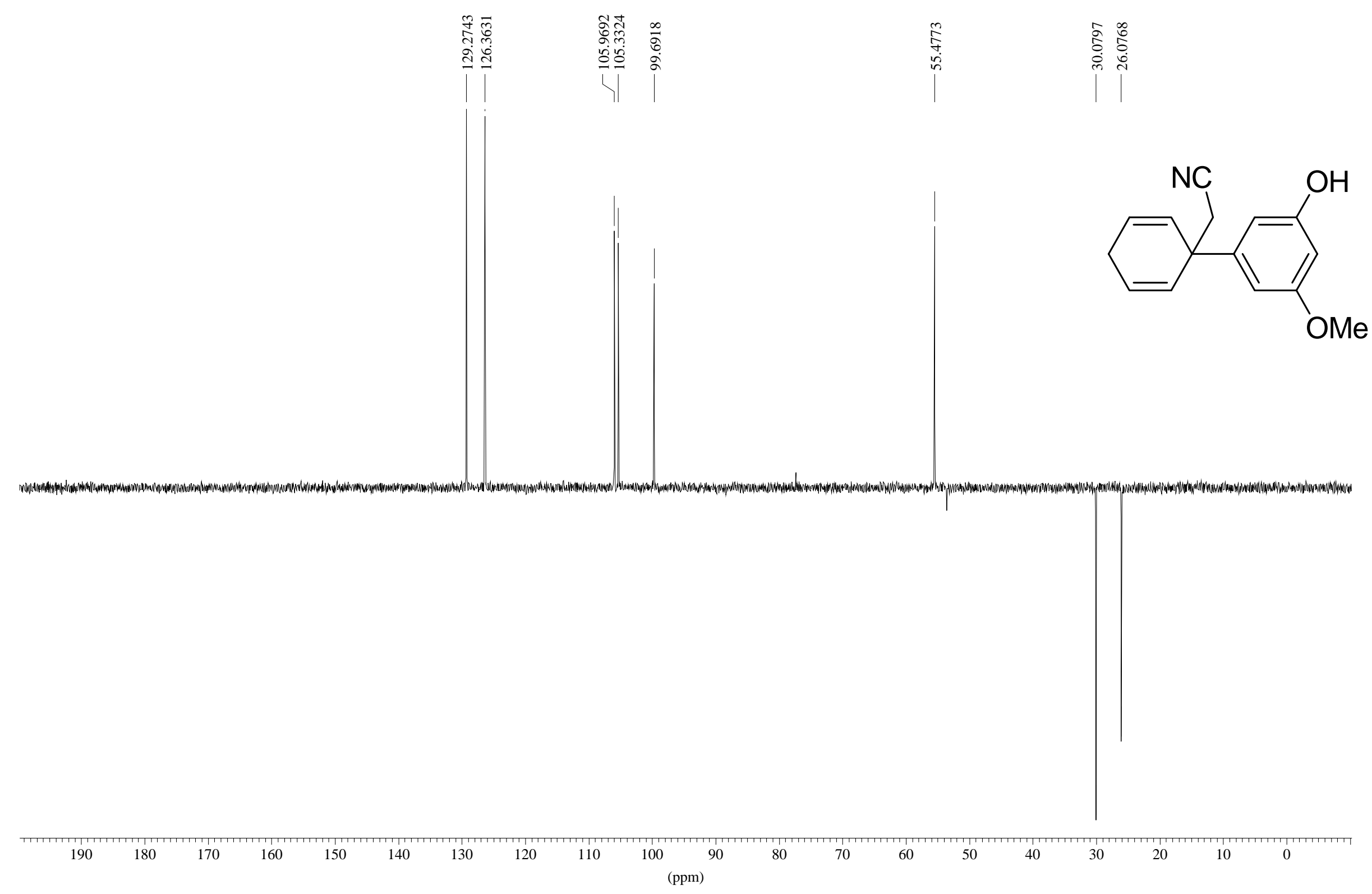




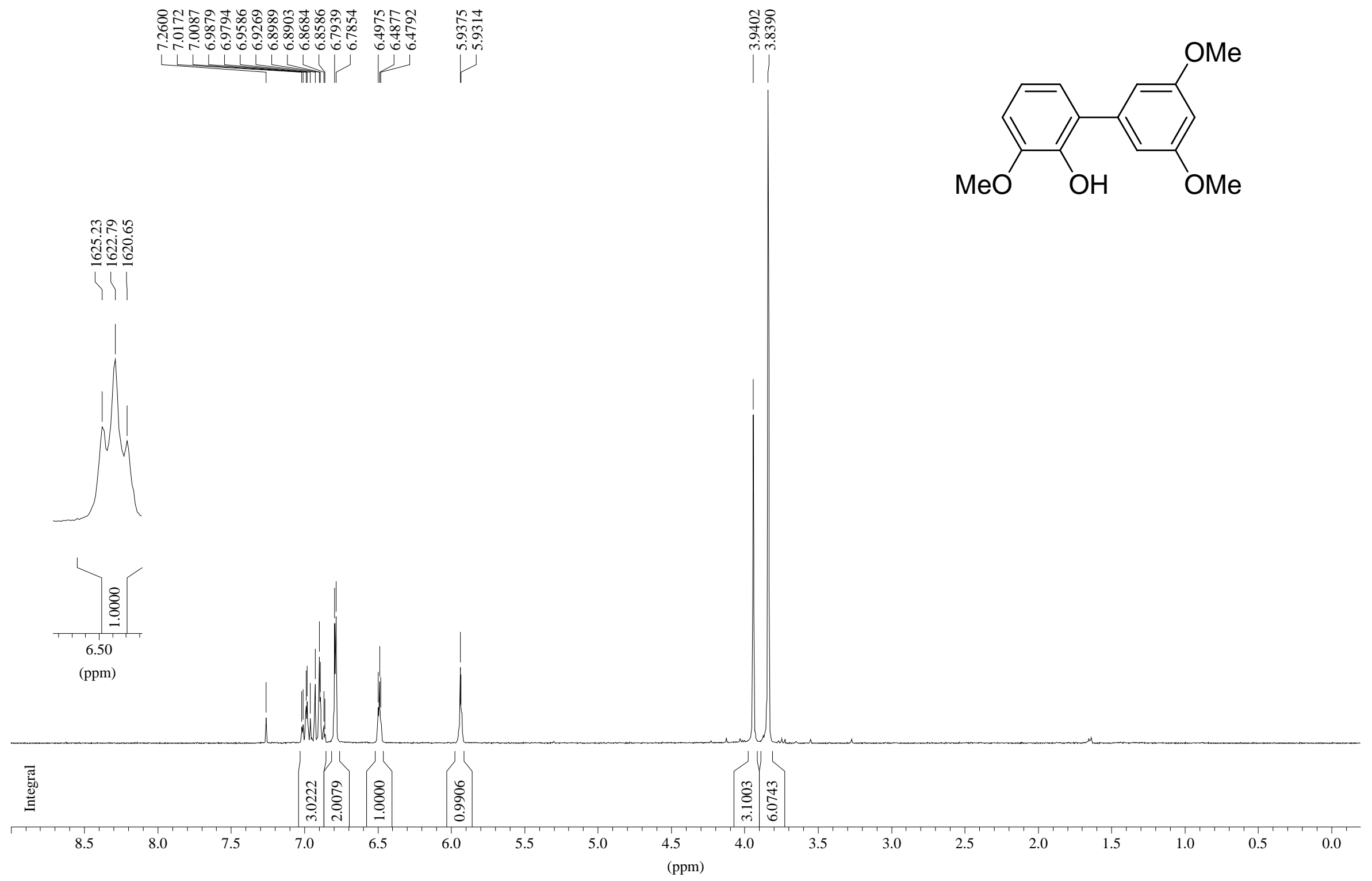




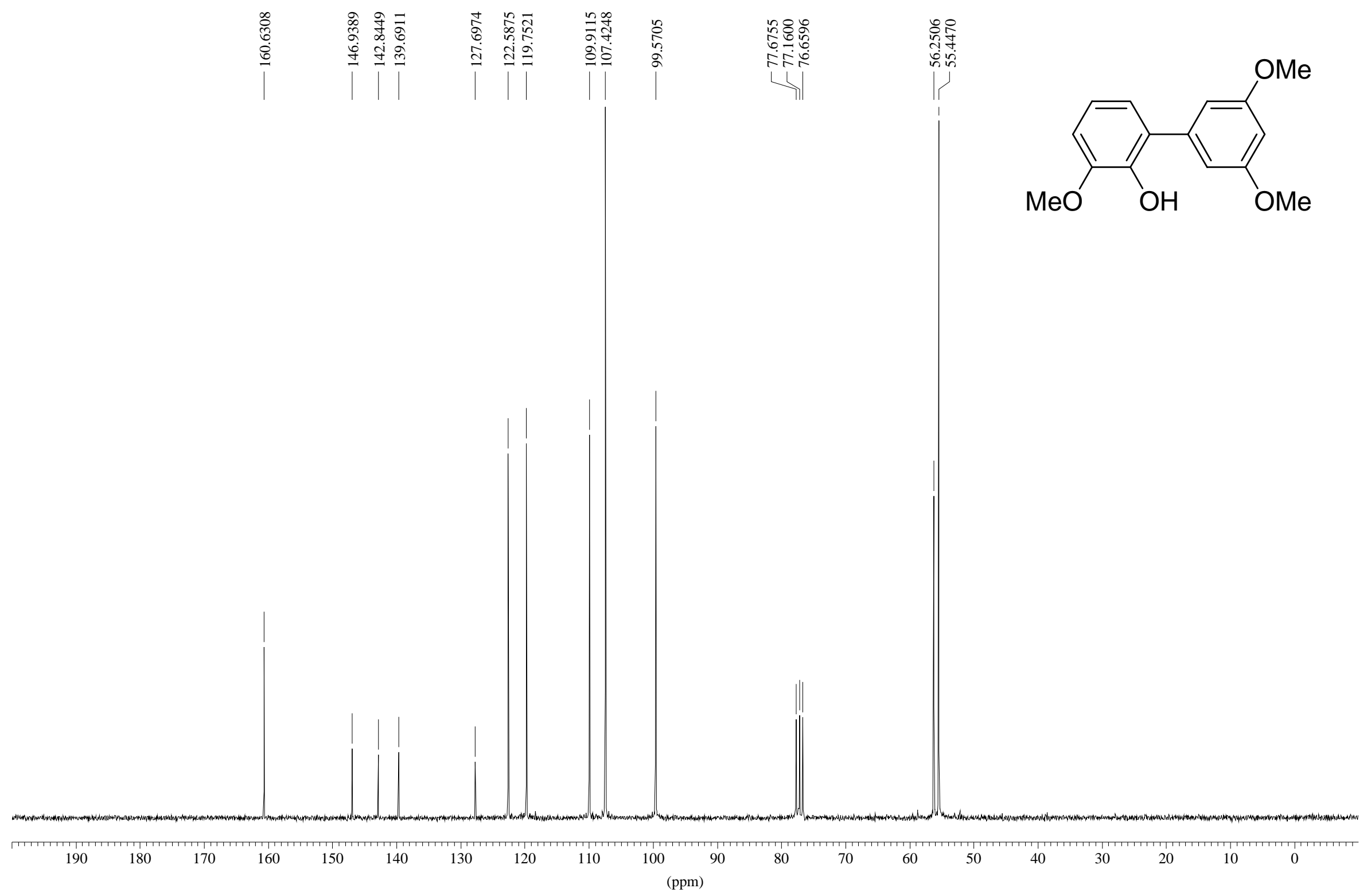




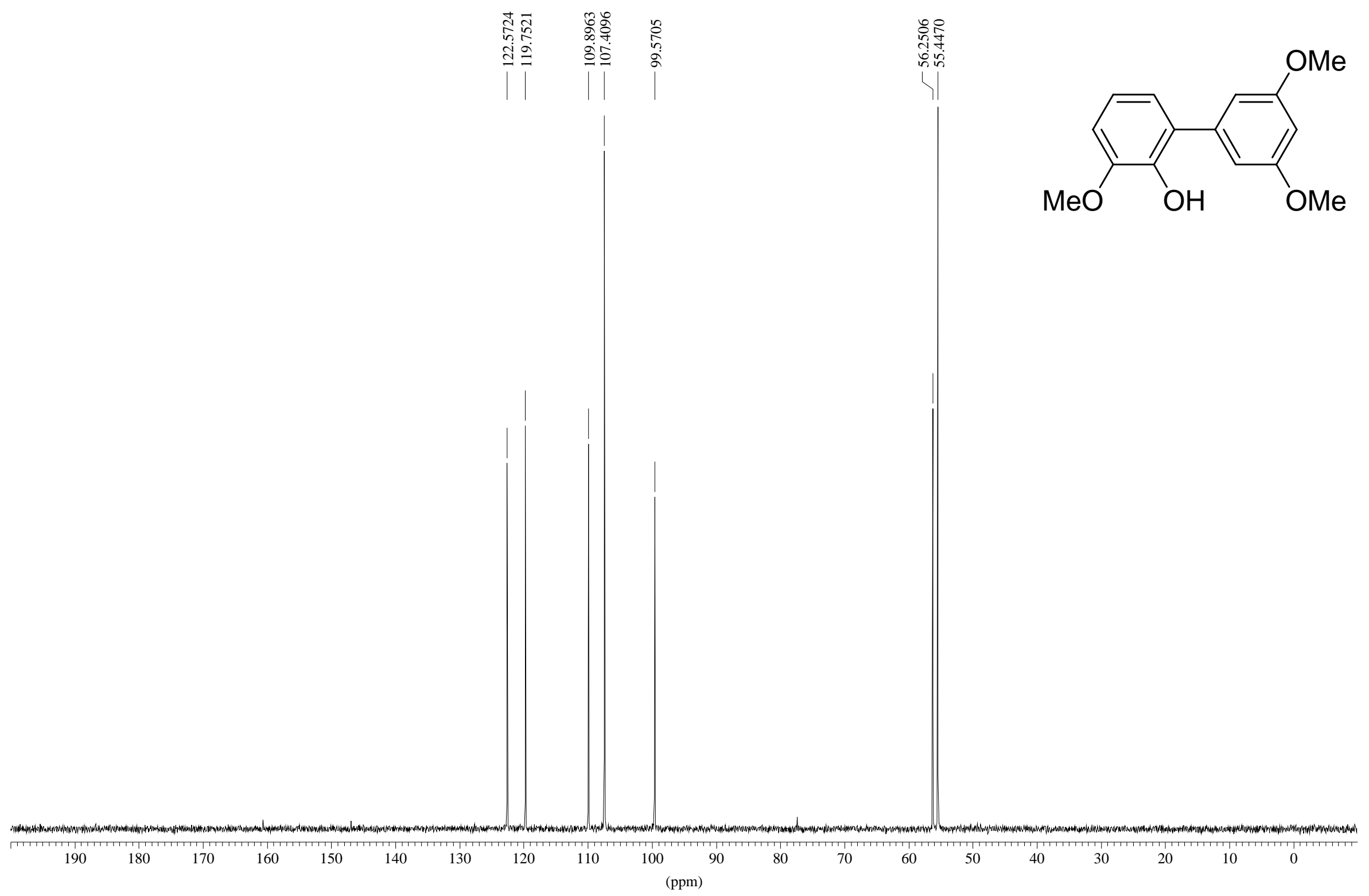




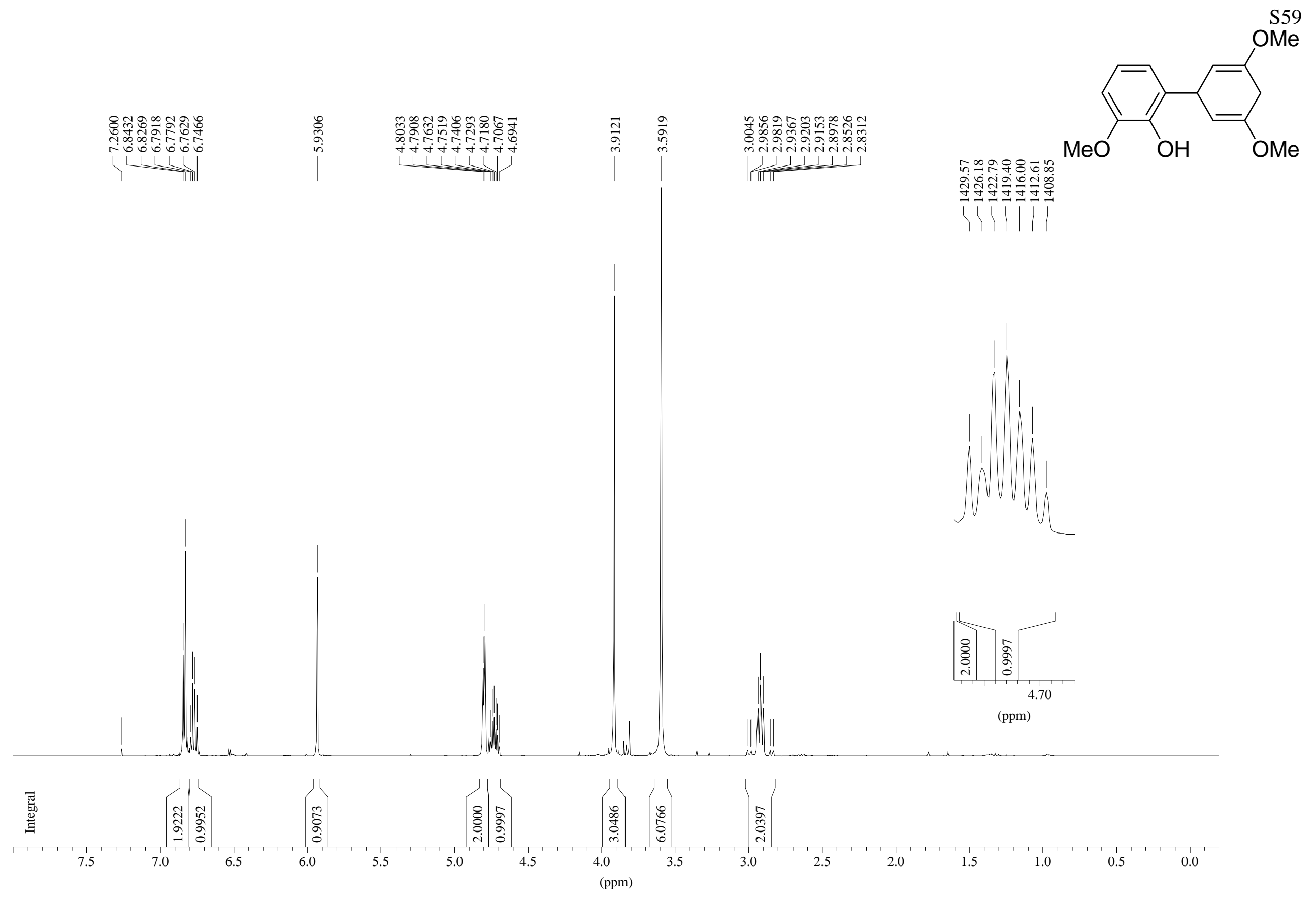




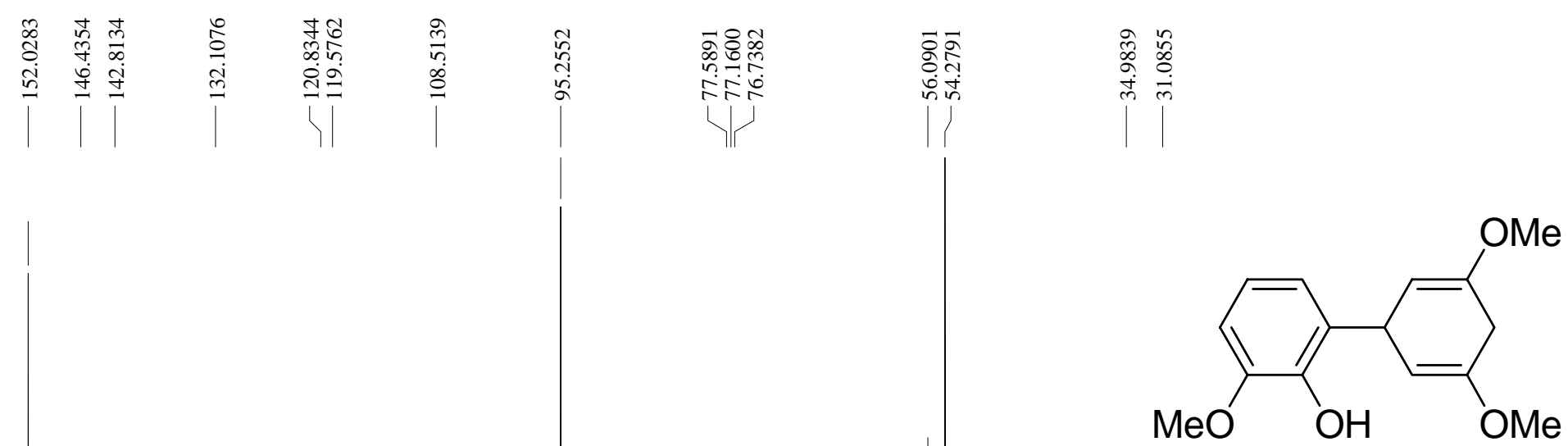




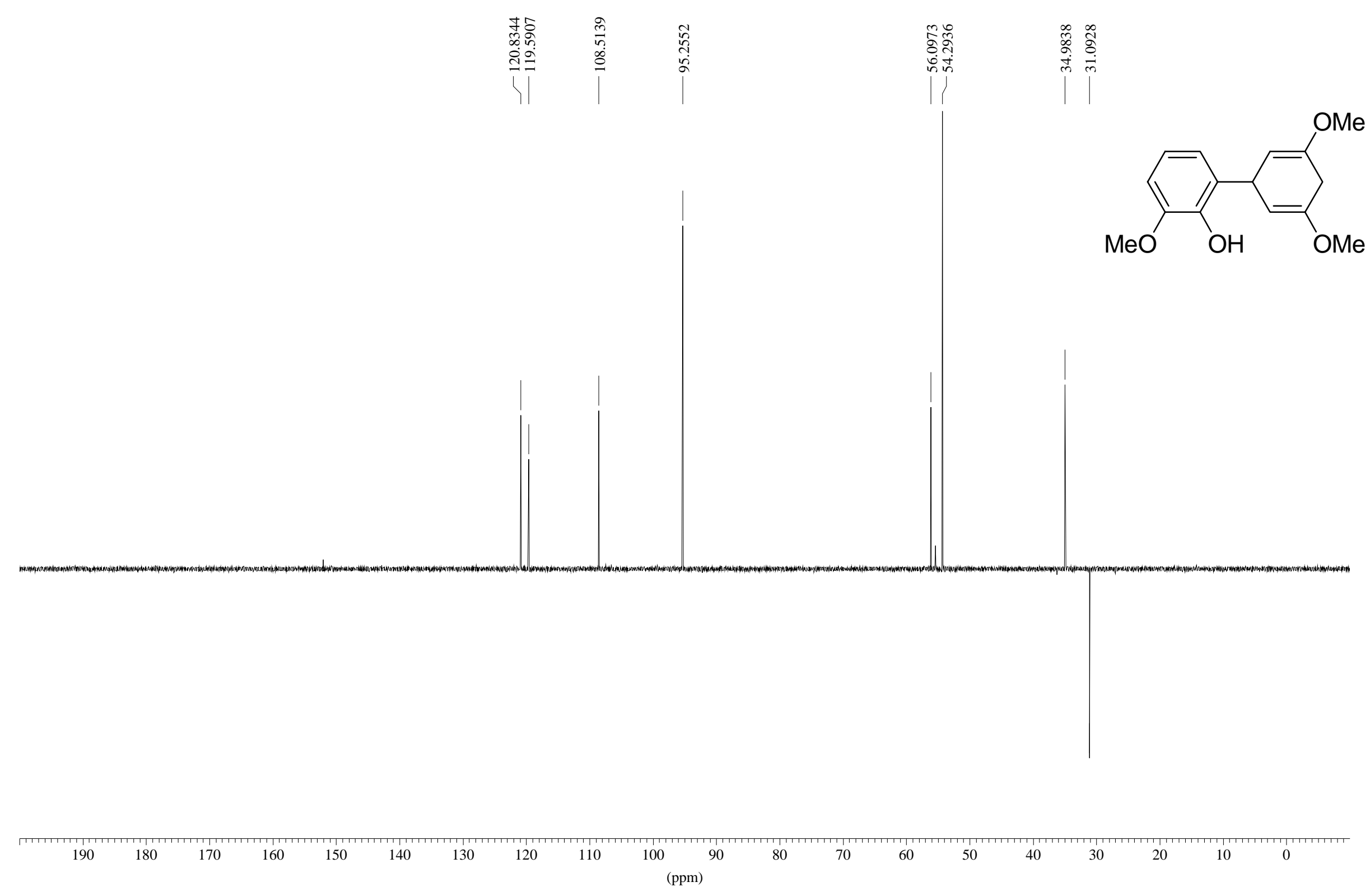




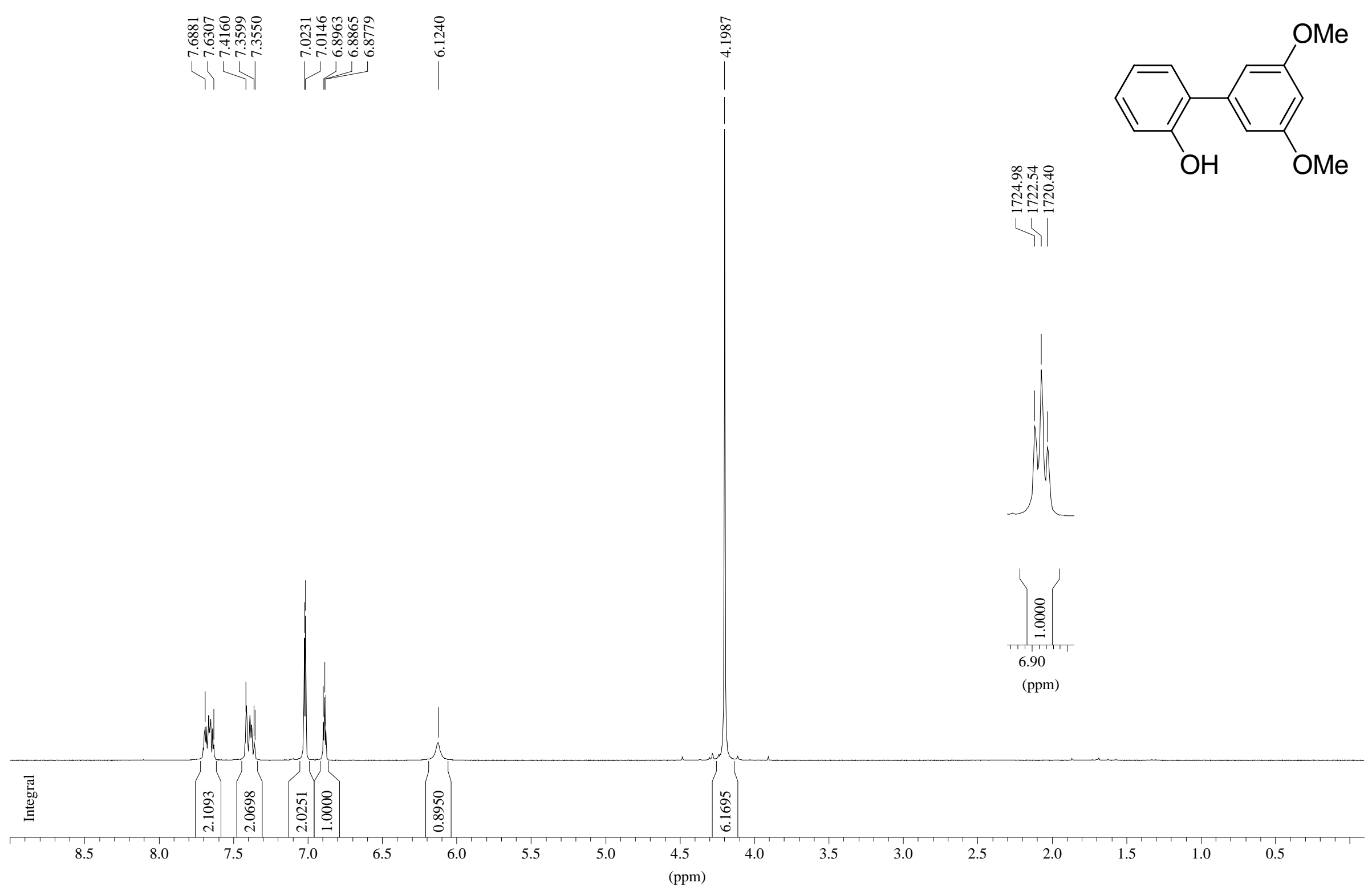




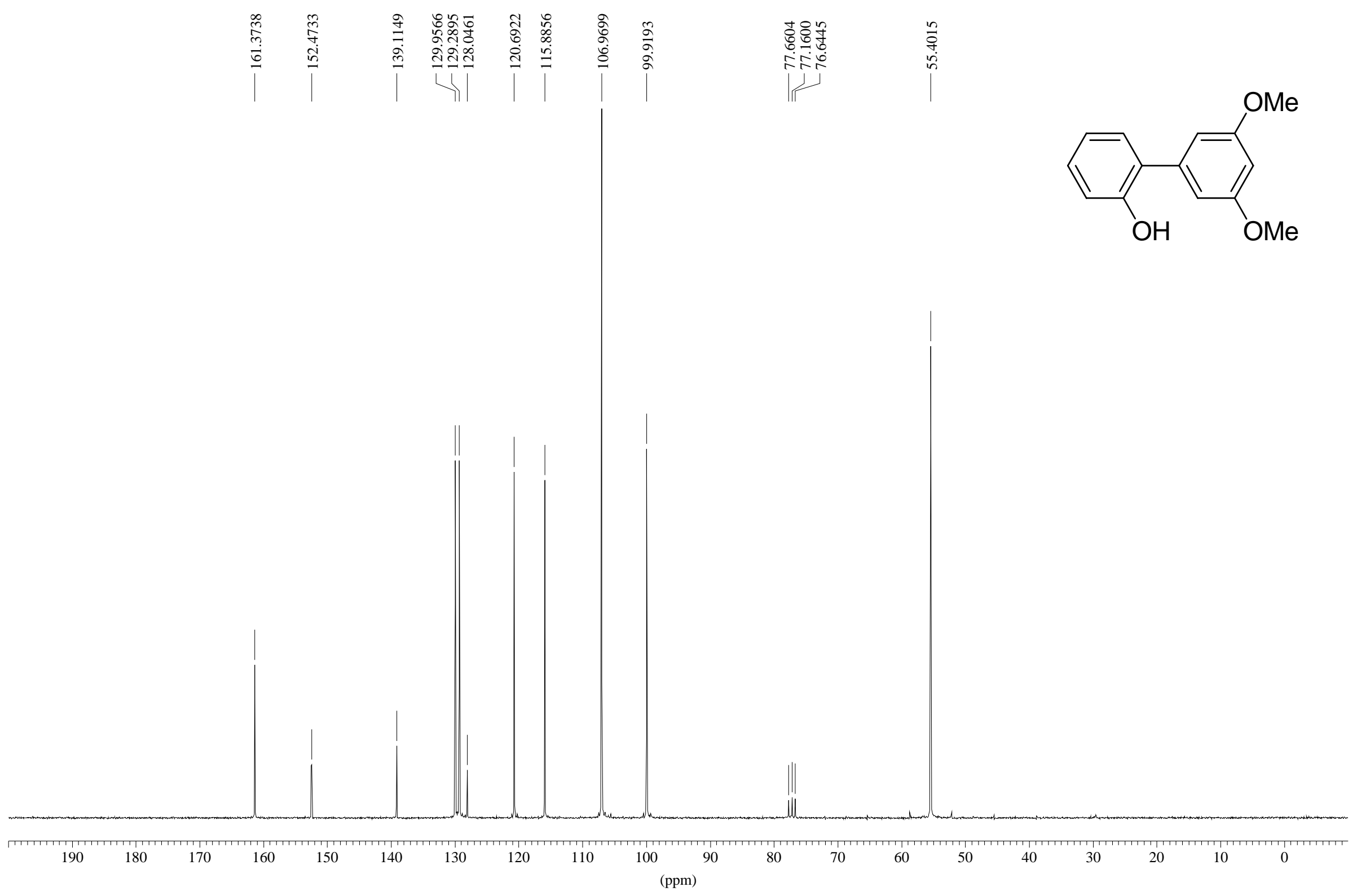




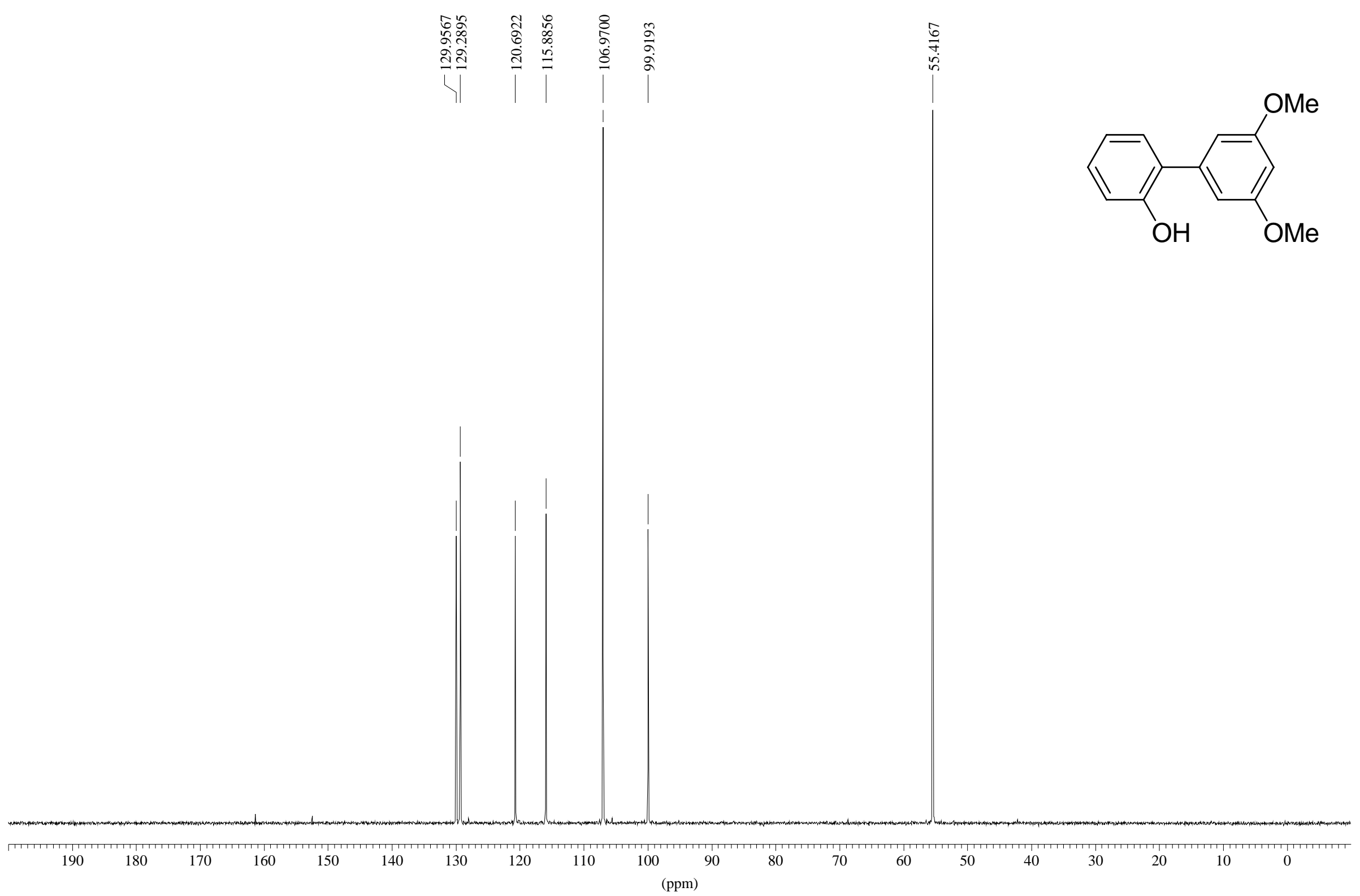




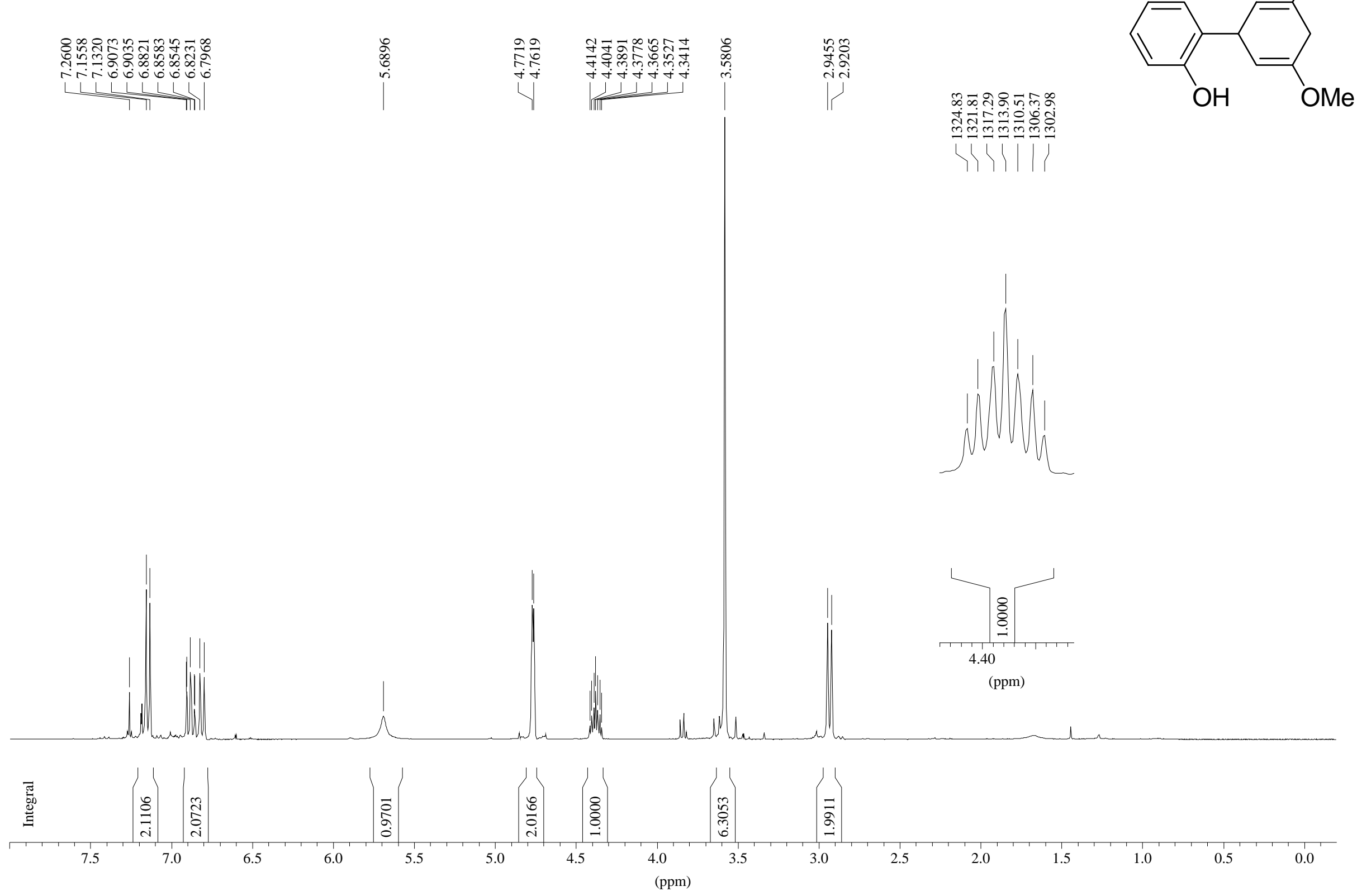




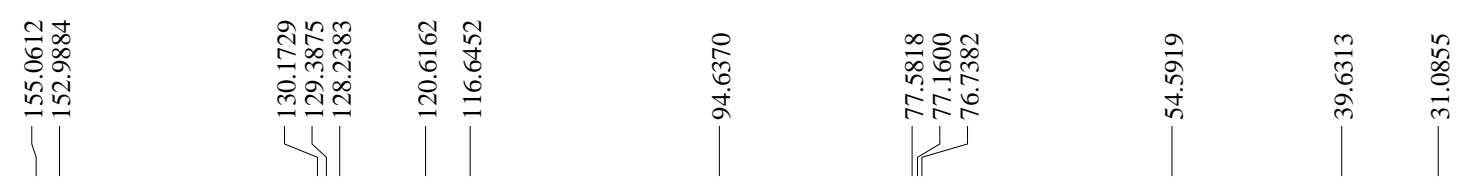

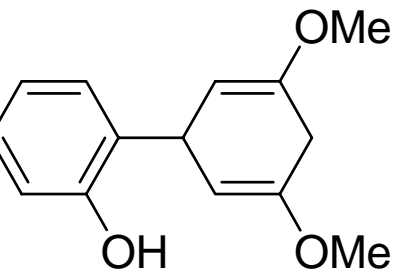

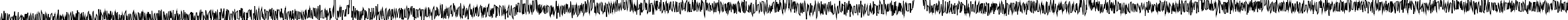




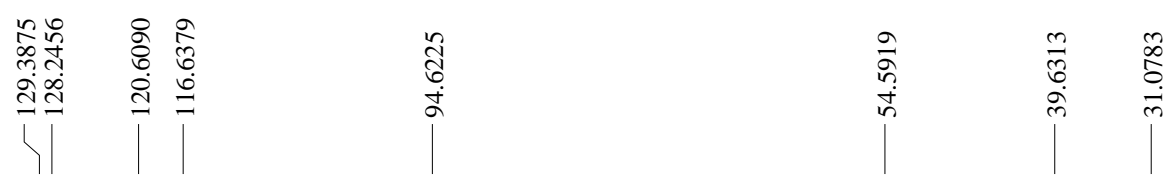

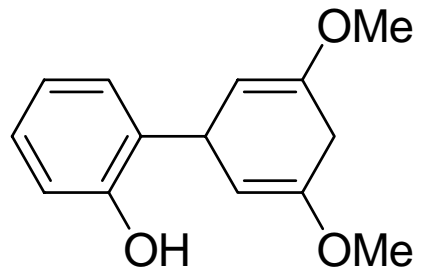




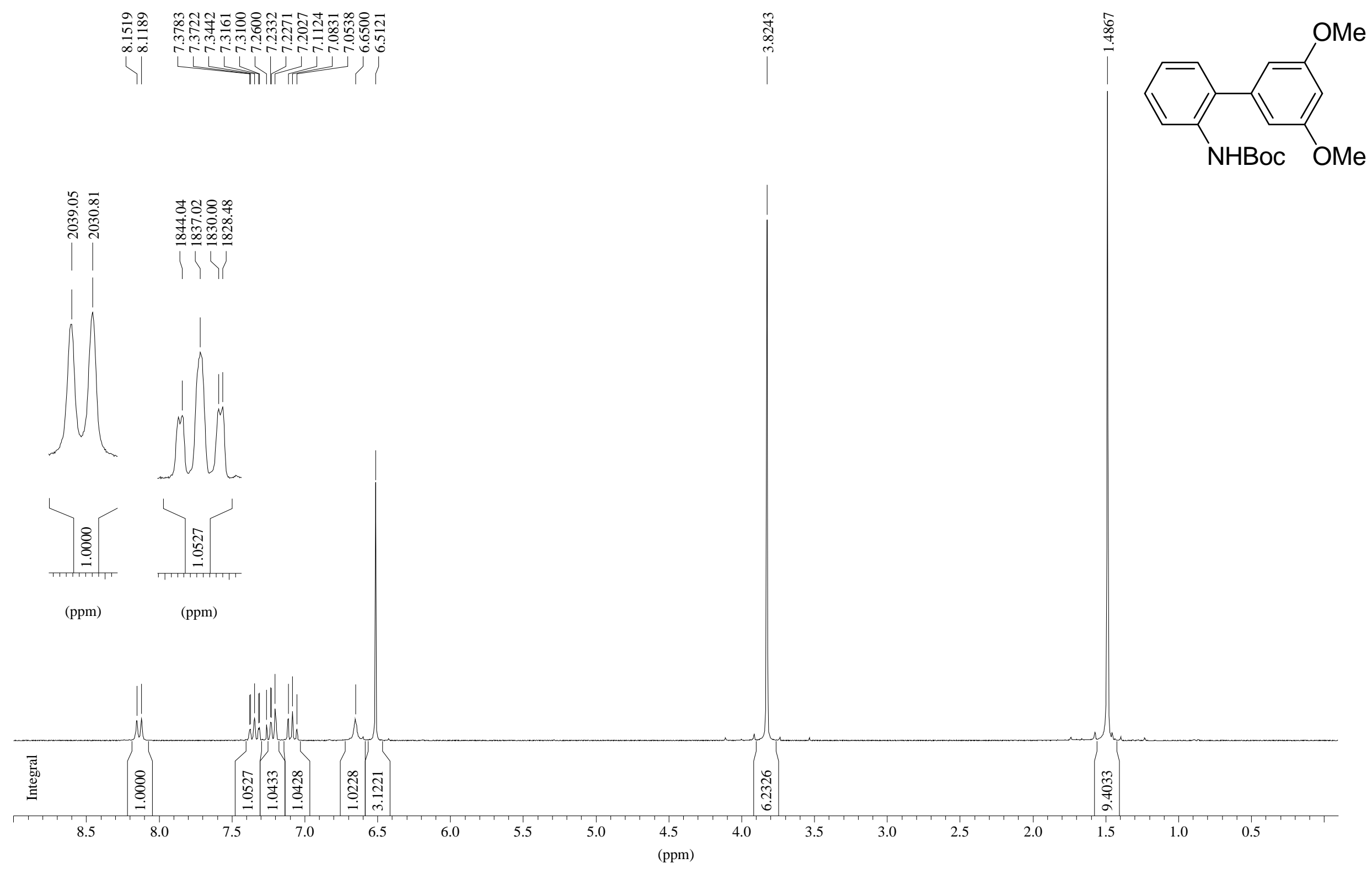




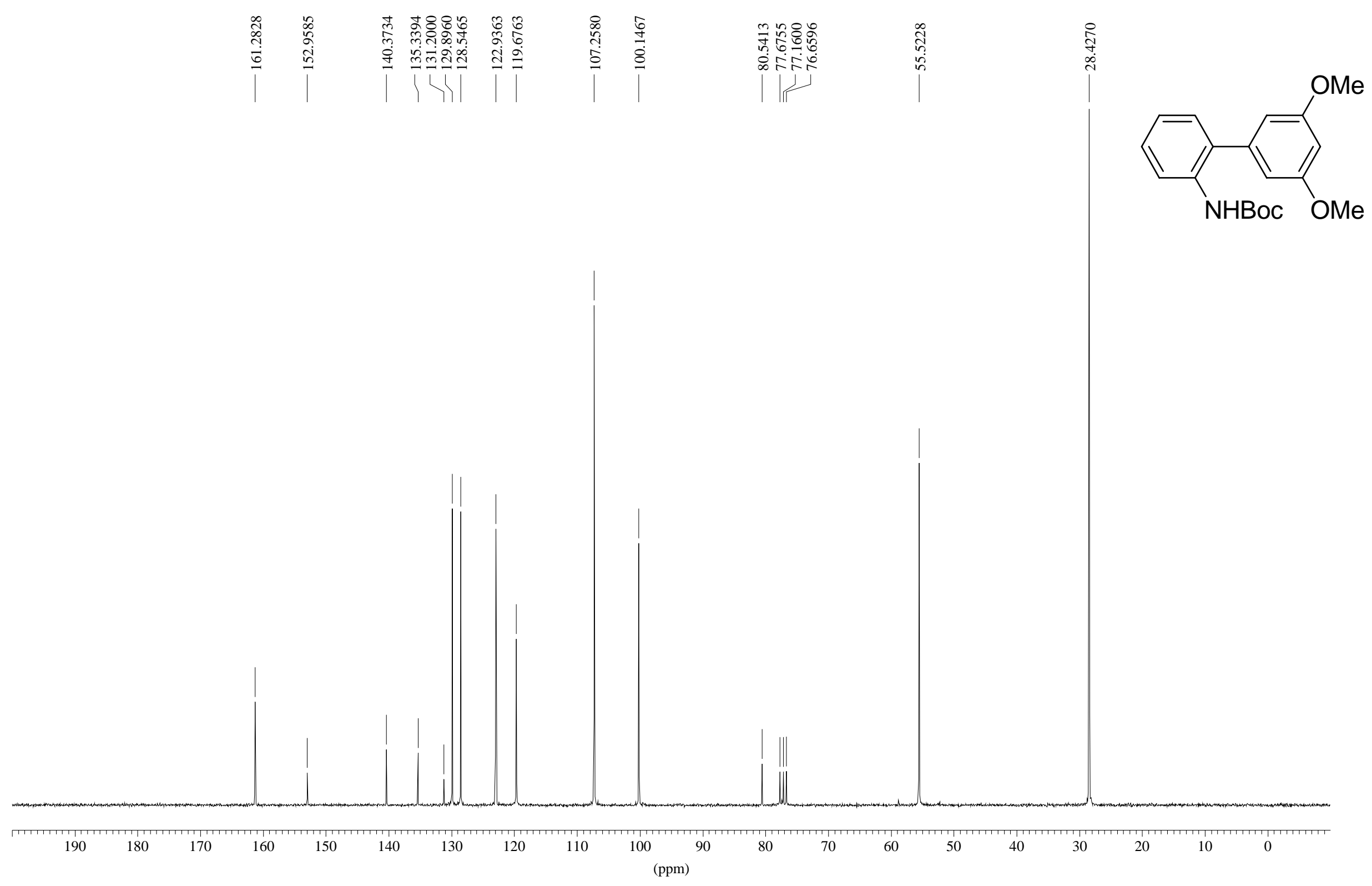




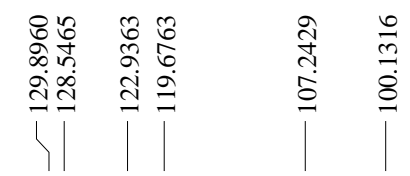

勇

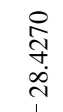

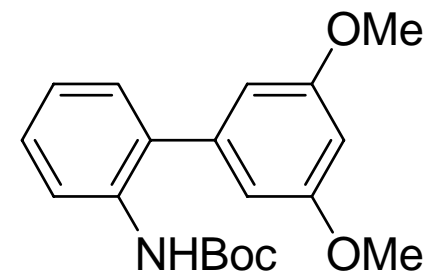

190 


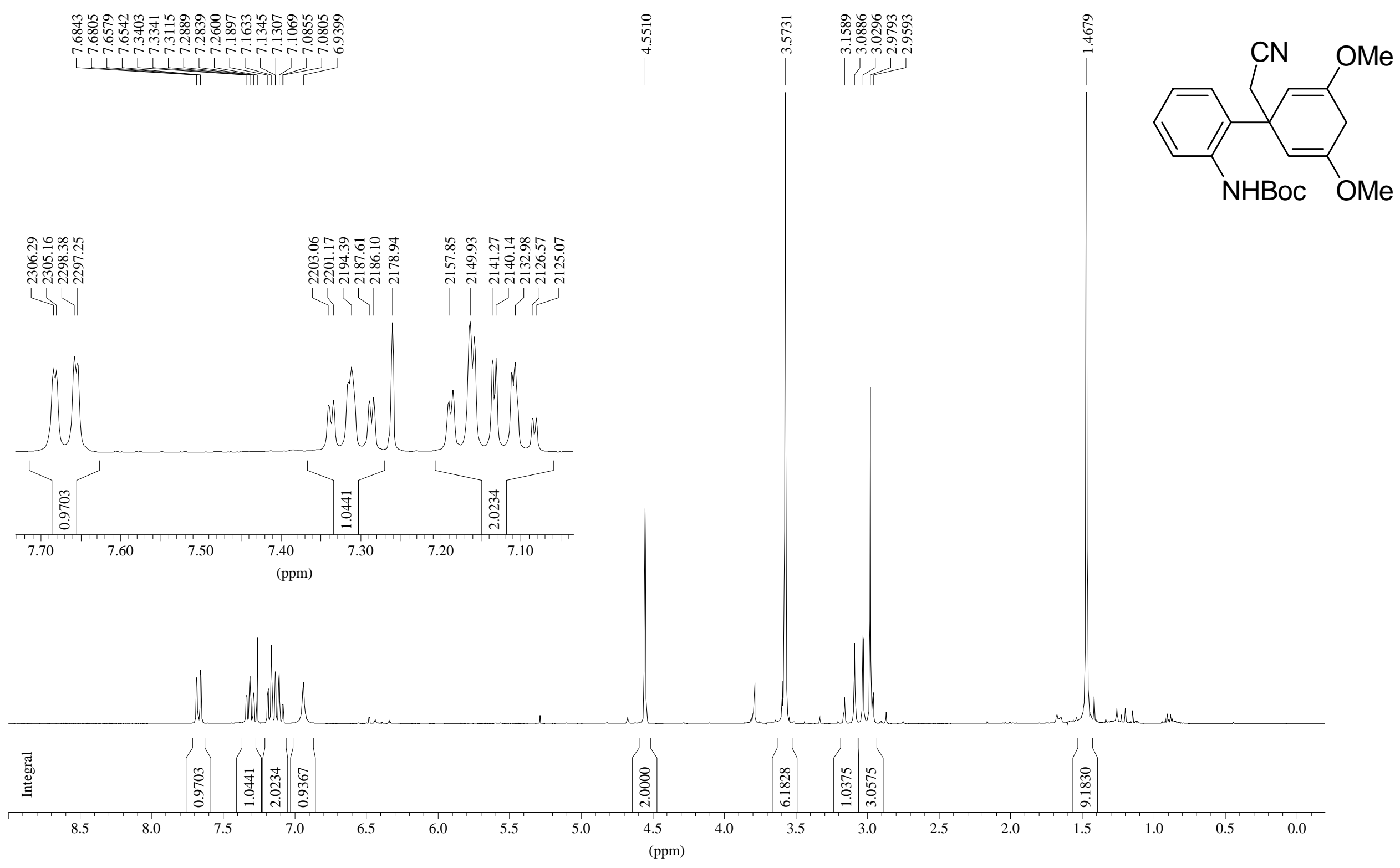




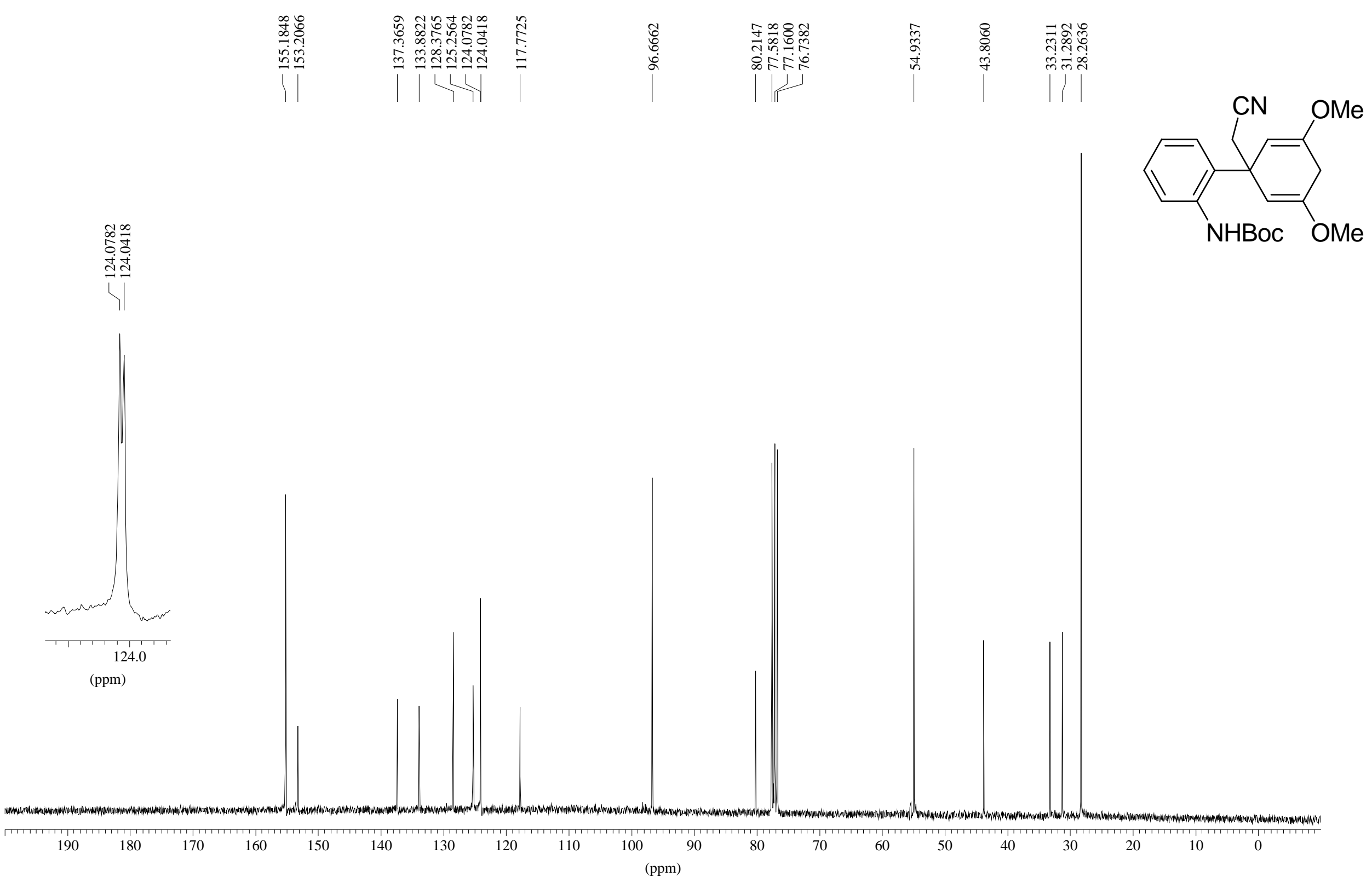




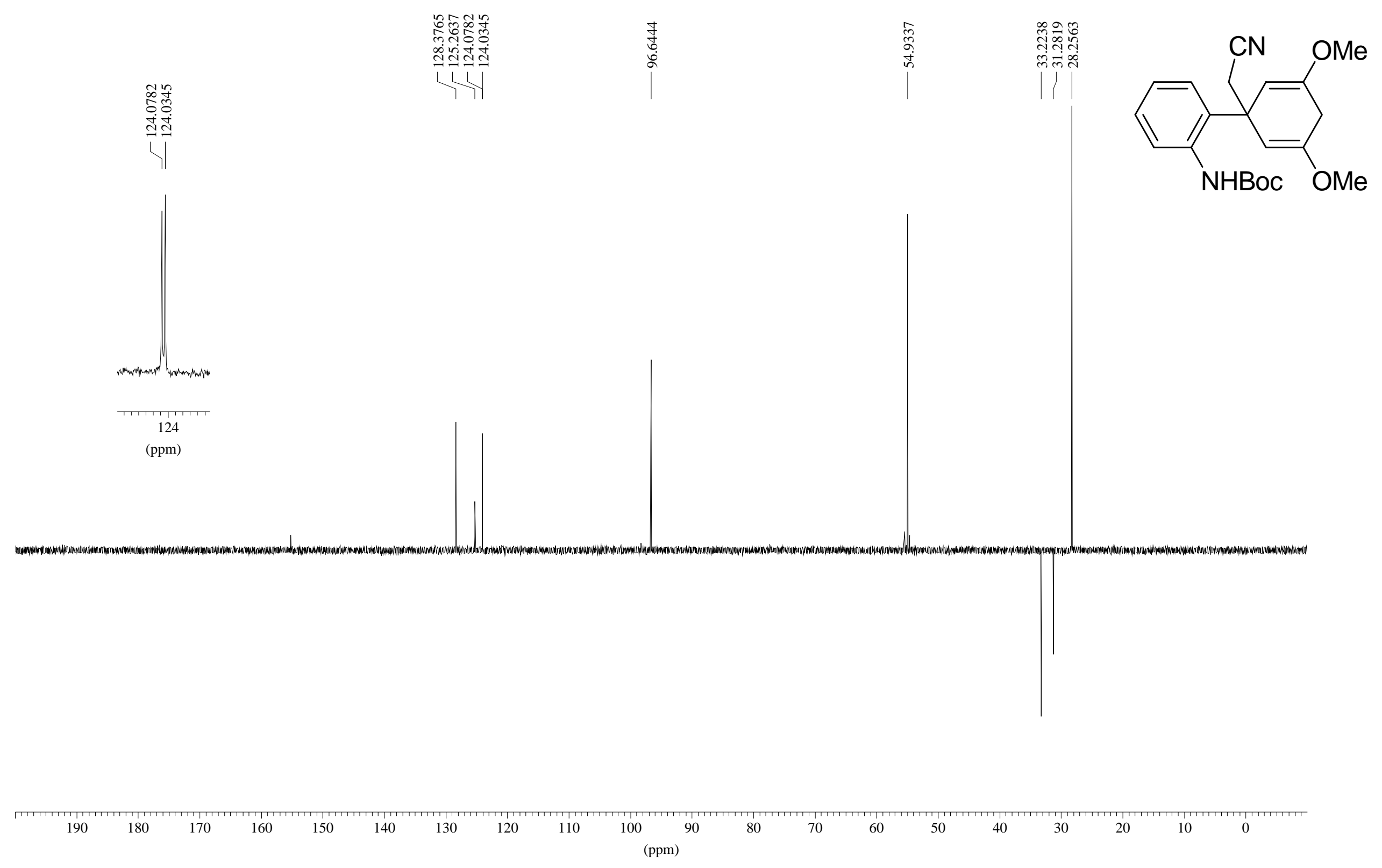




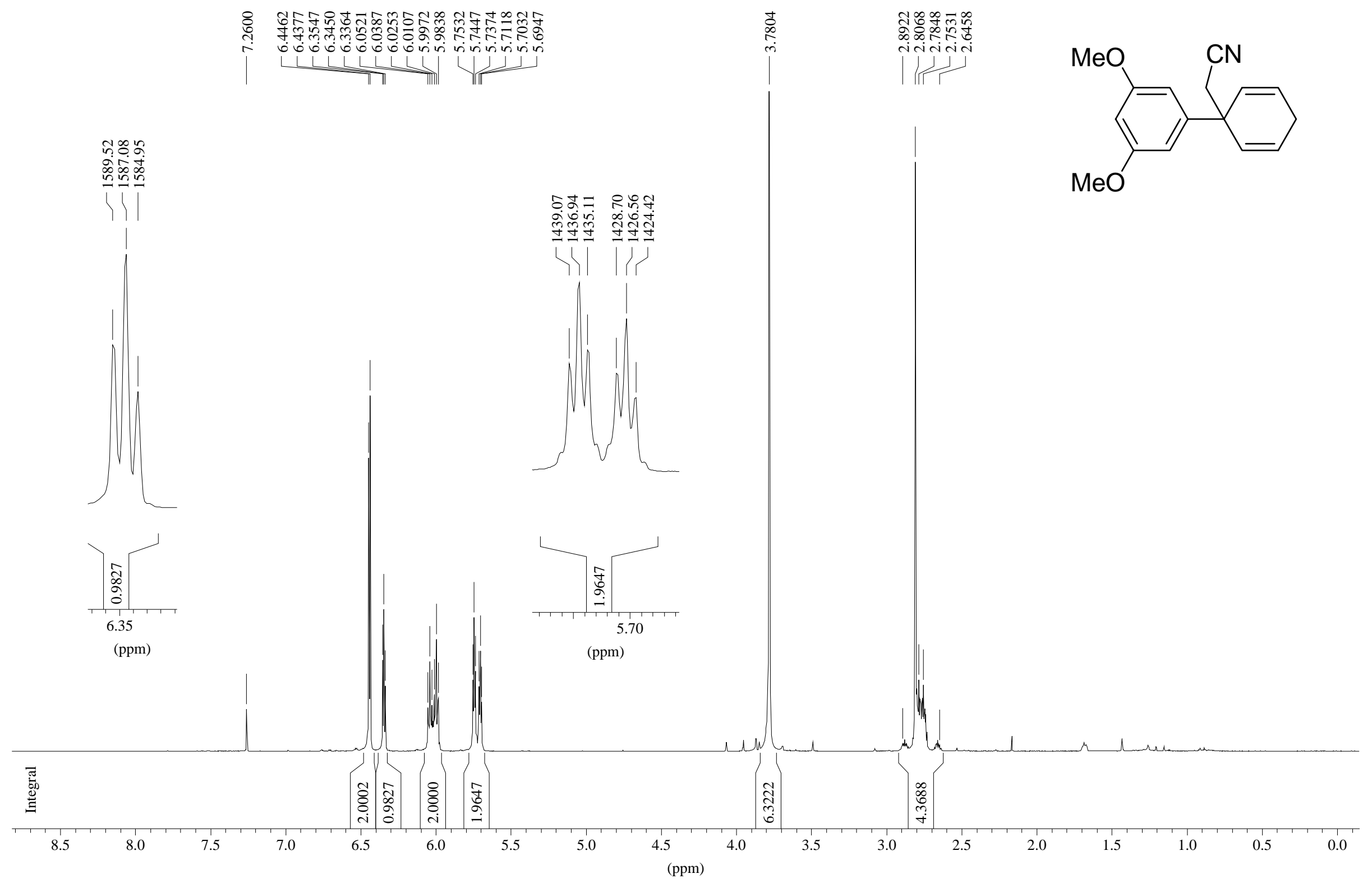




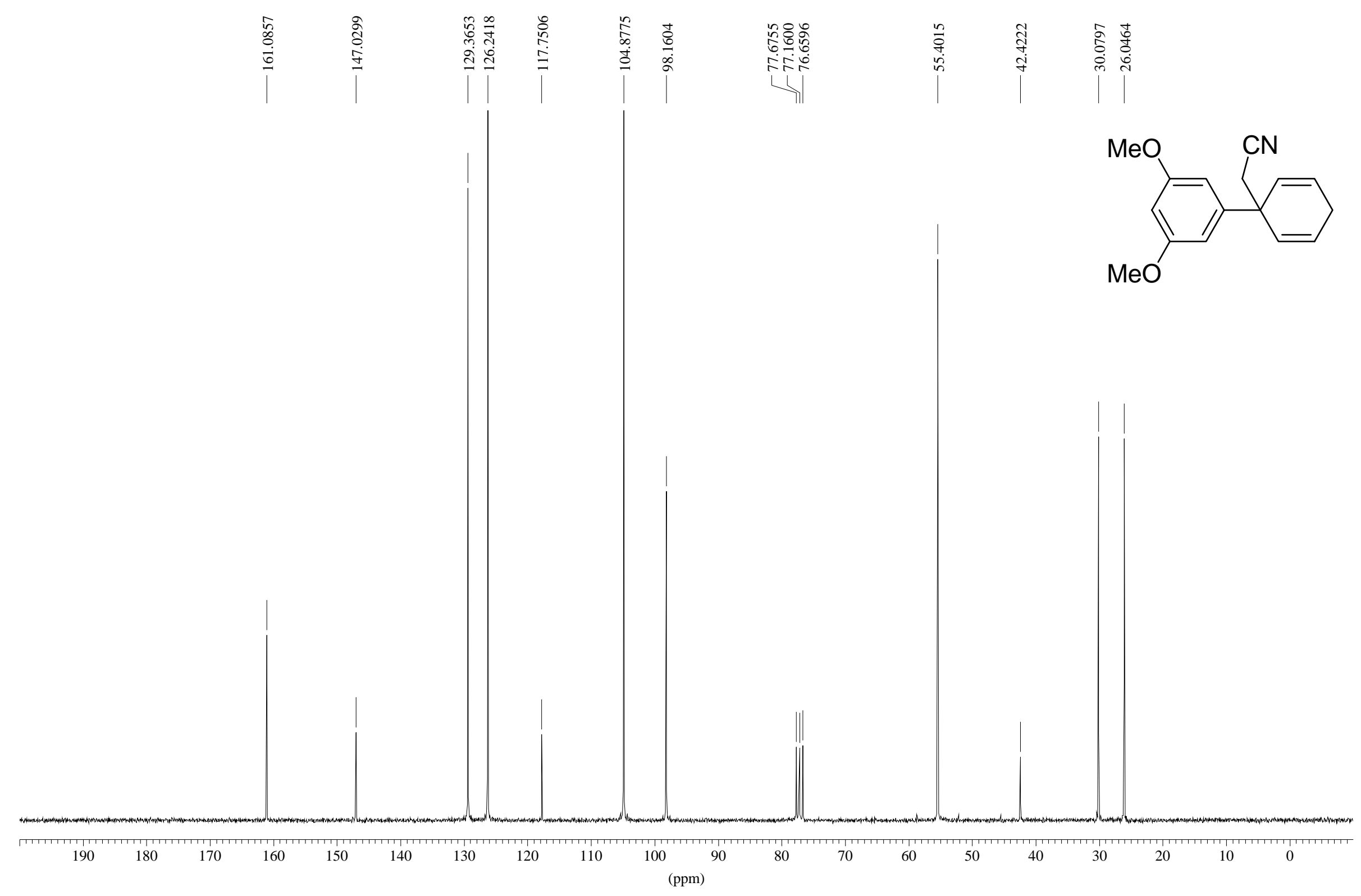




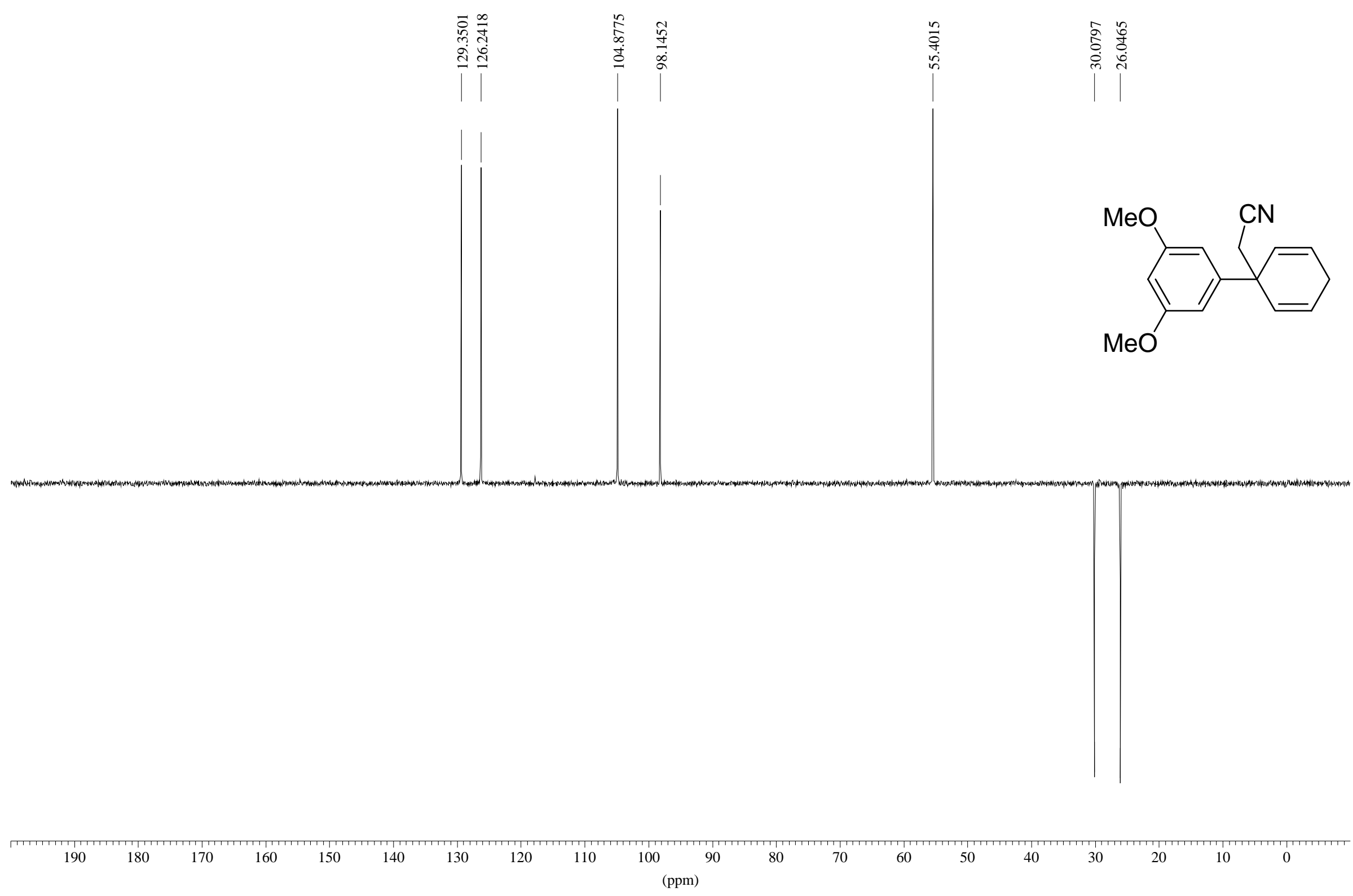




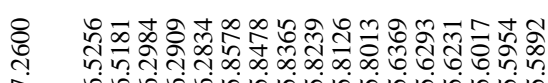

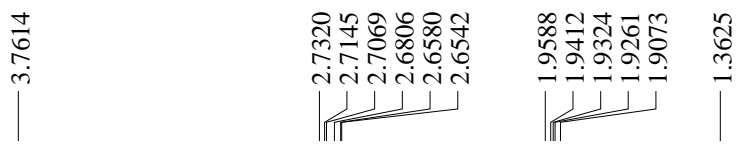

L
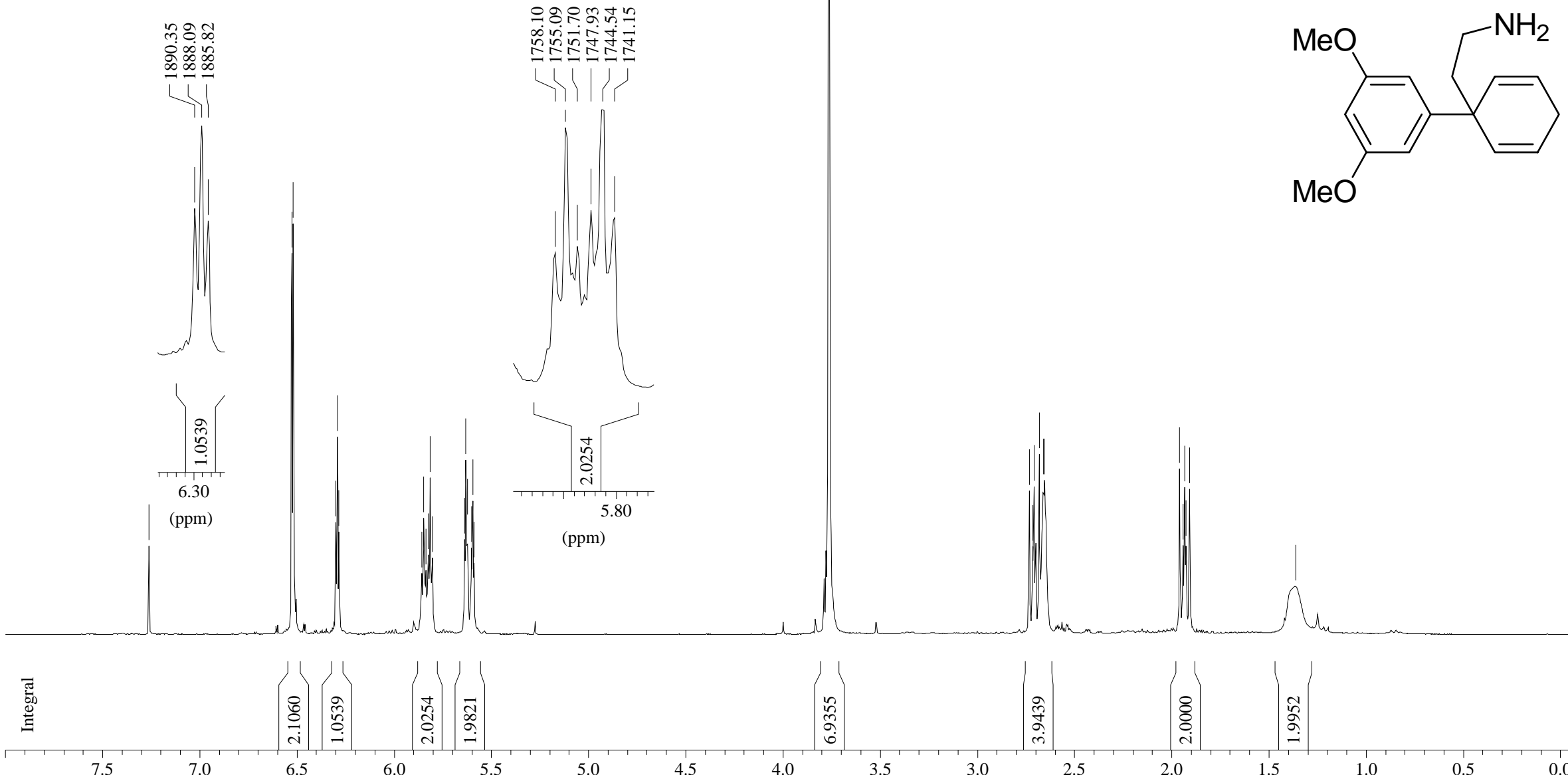

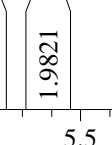

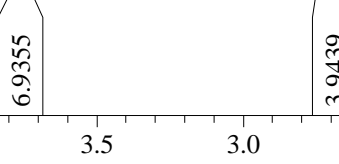

(ppm) 


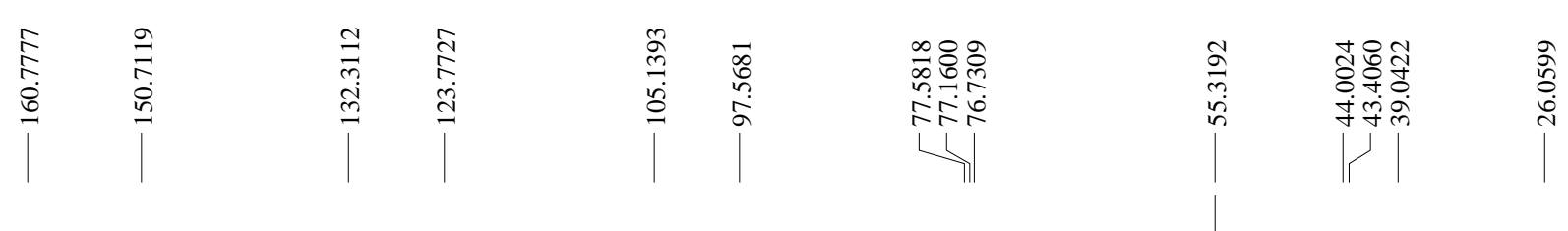
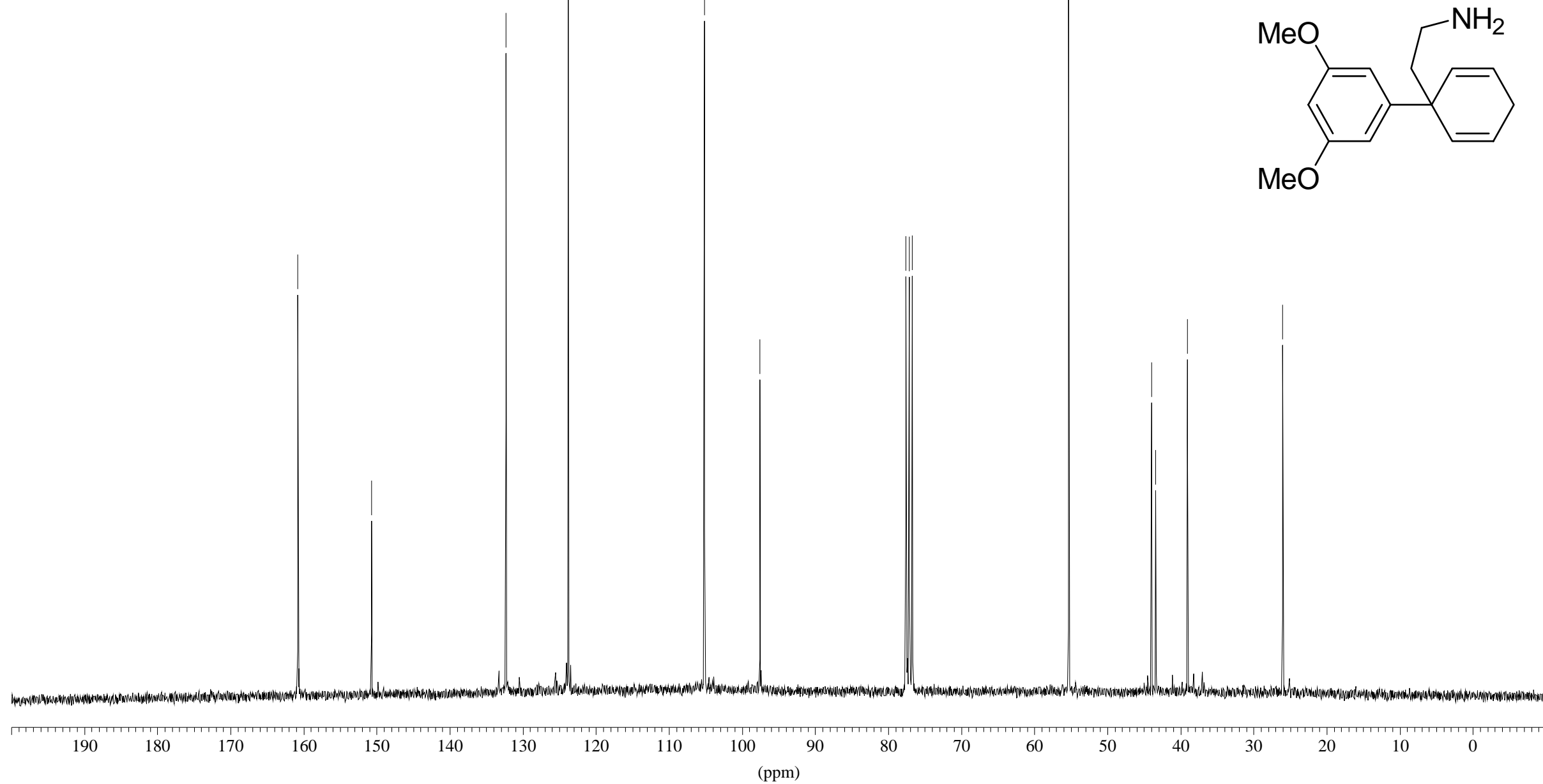


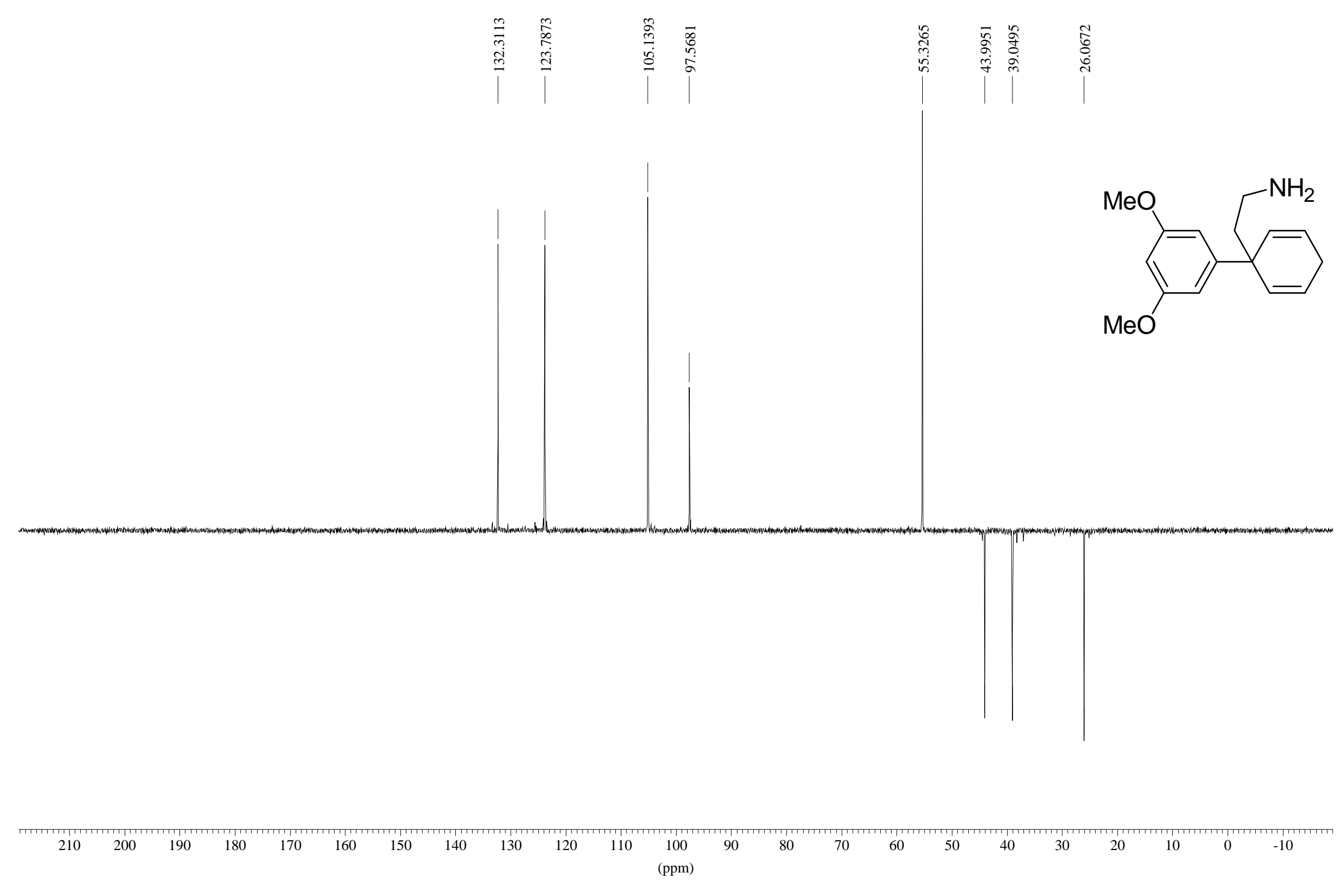



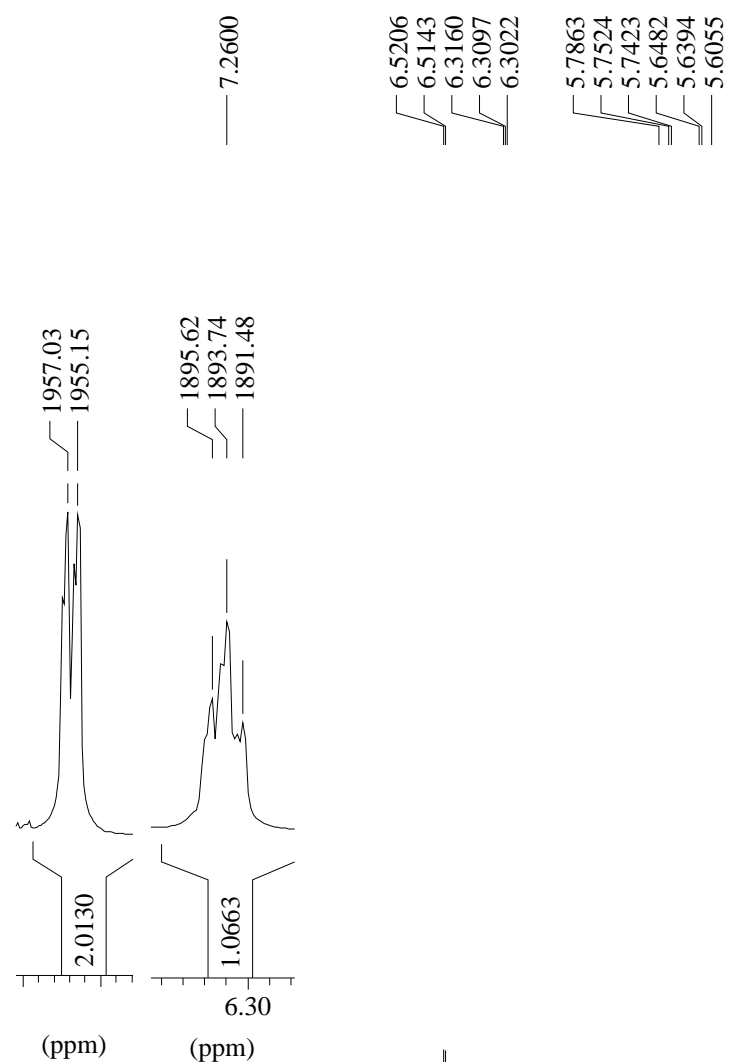

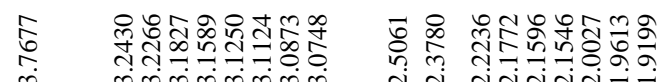

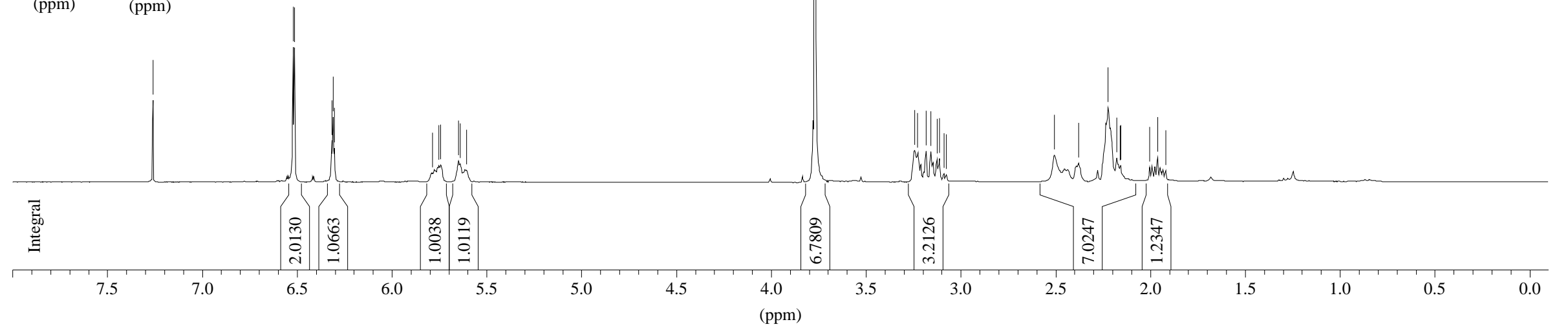




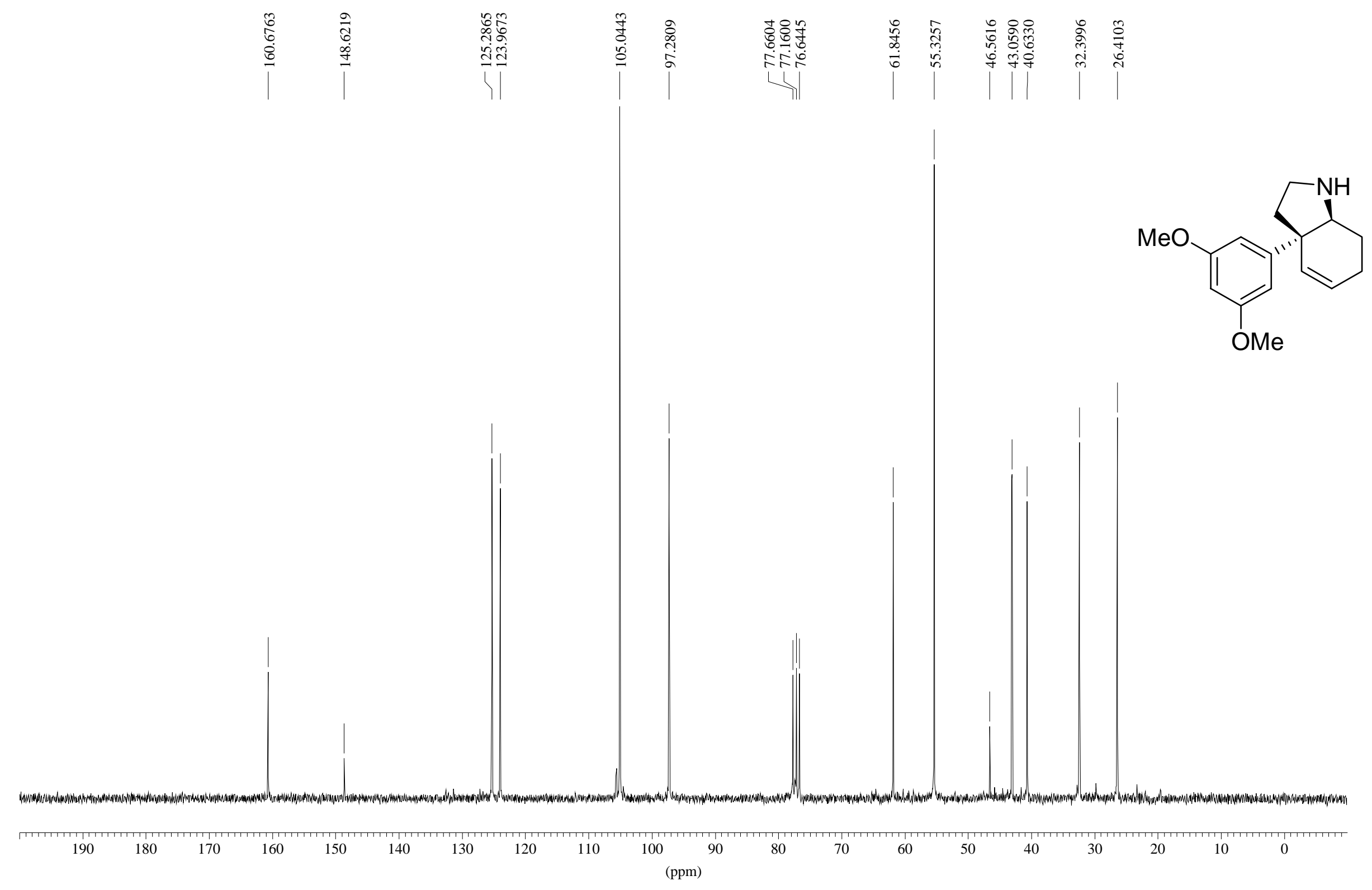




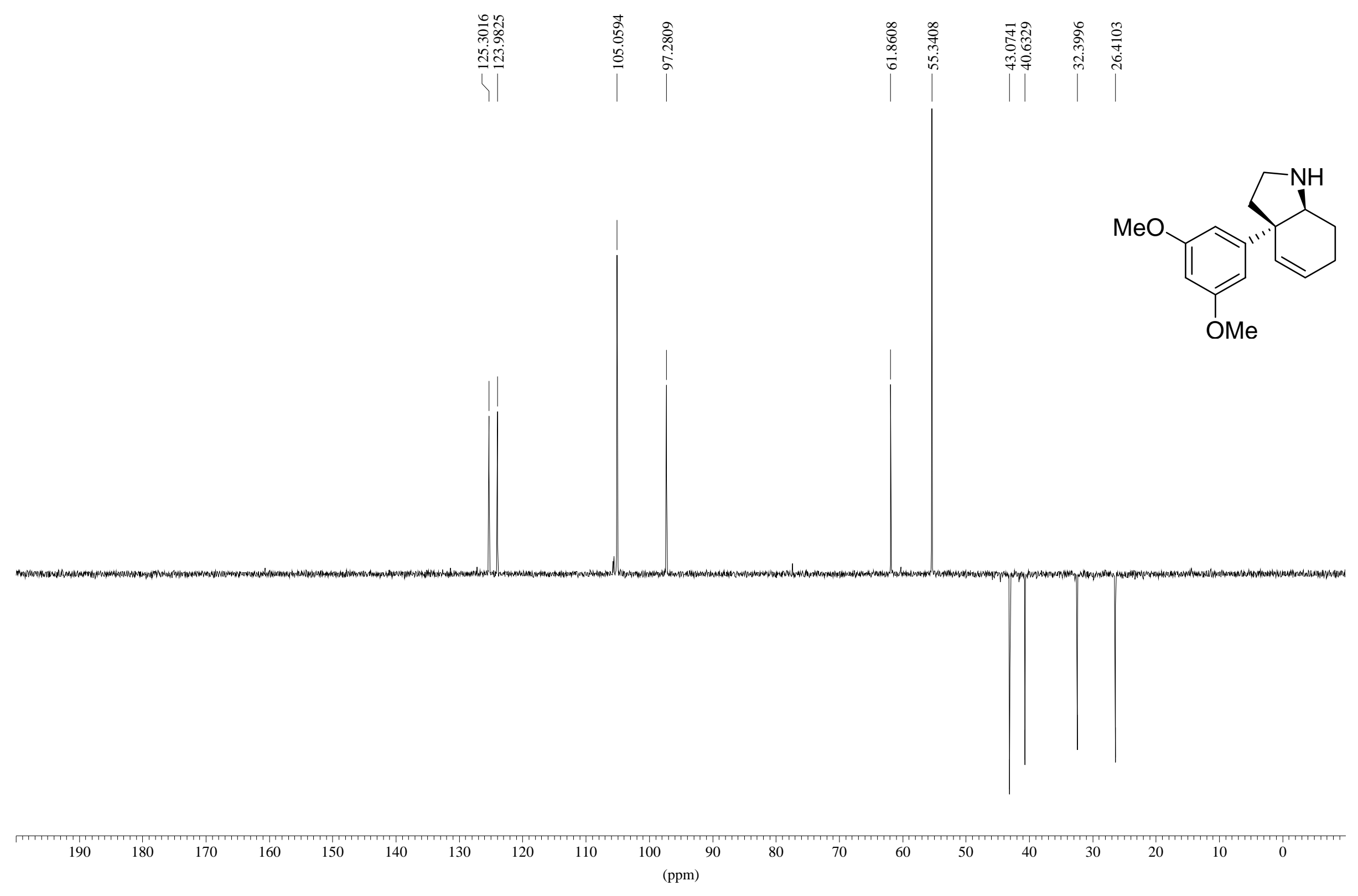




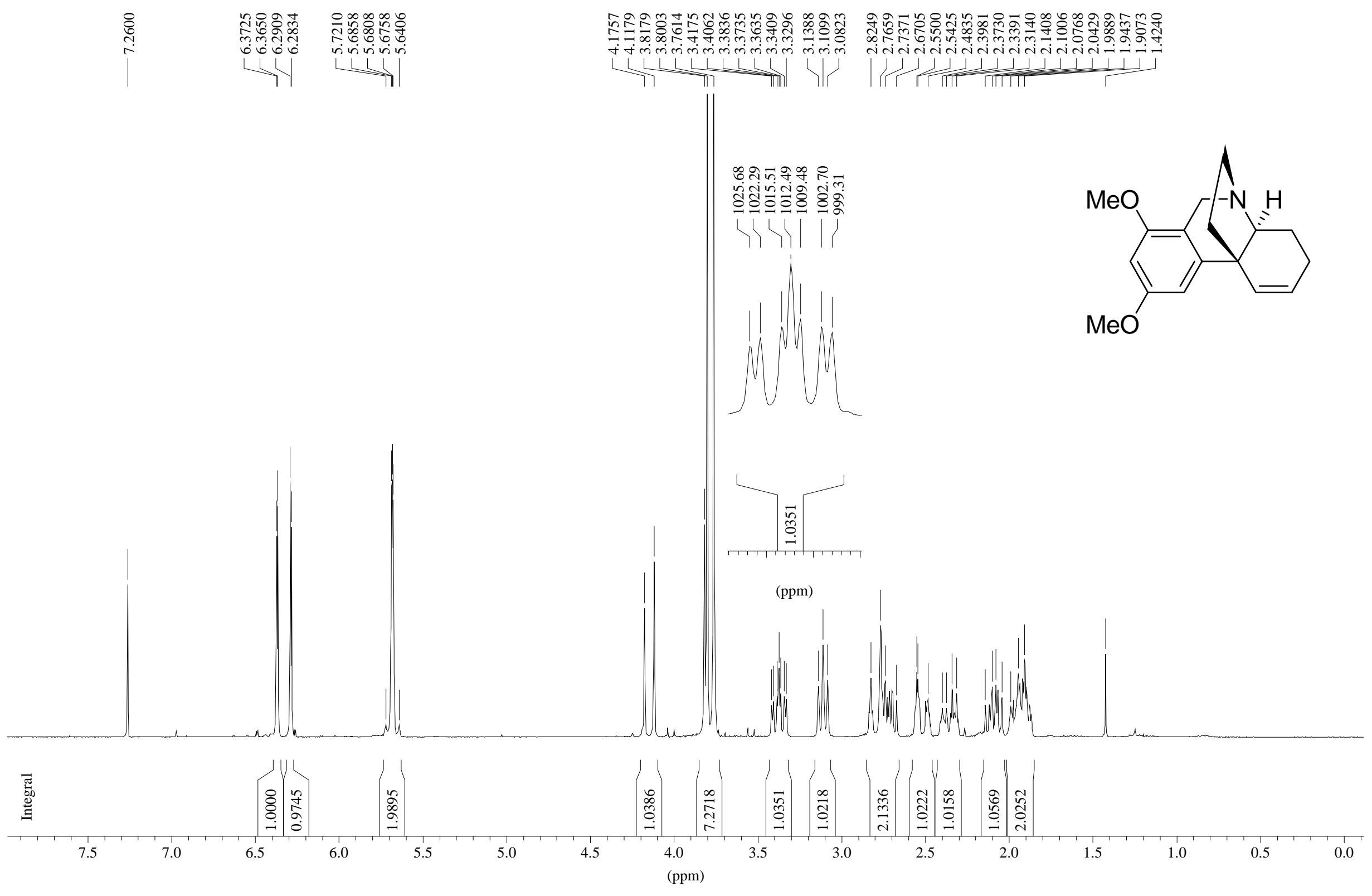




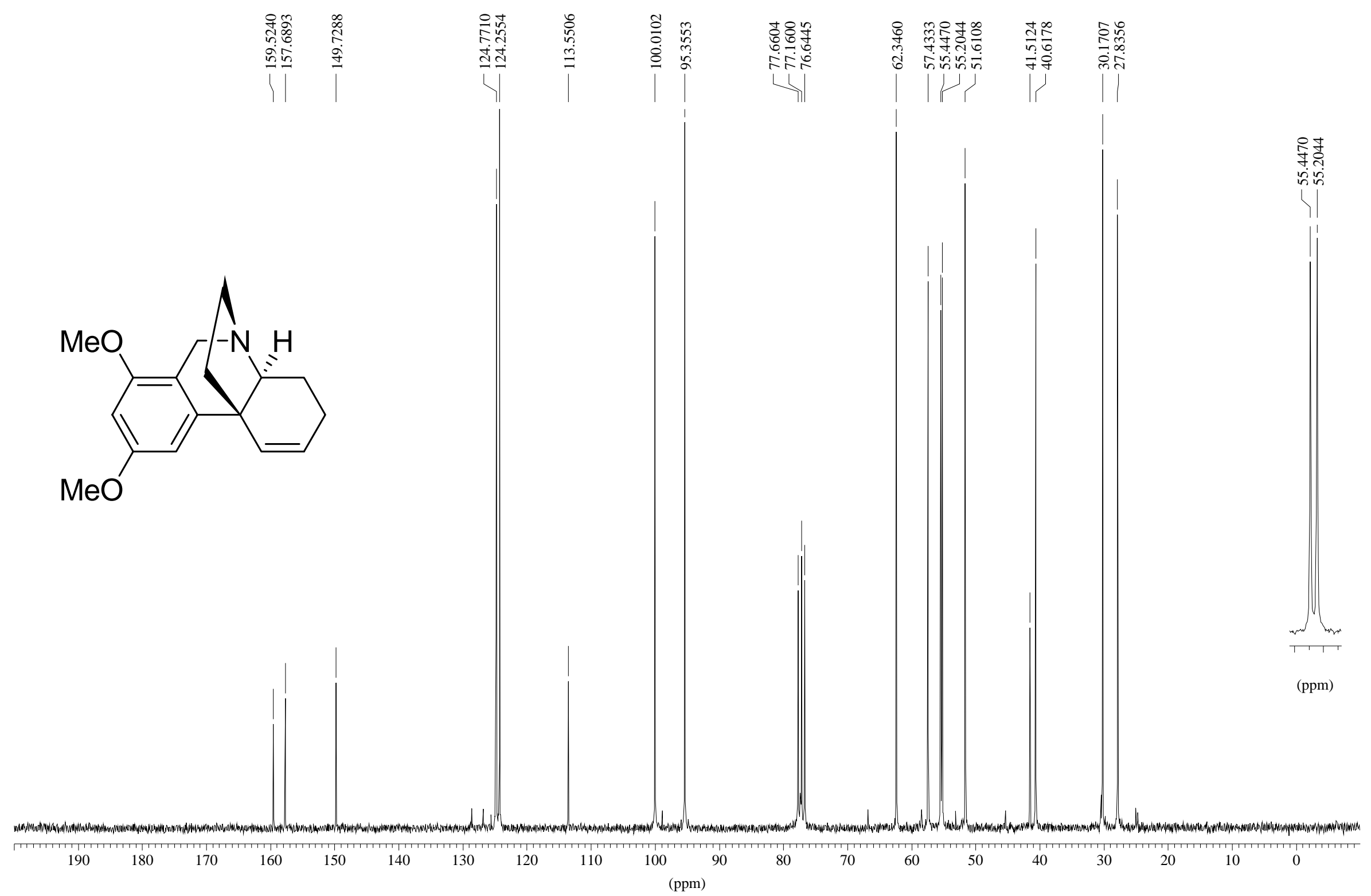




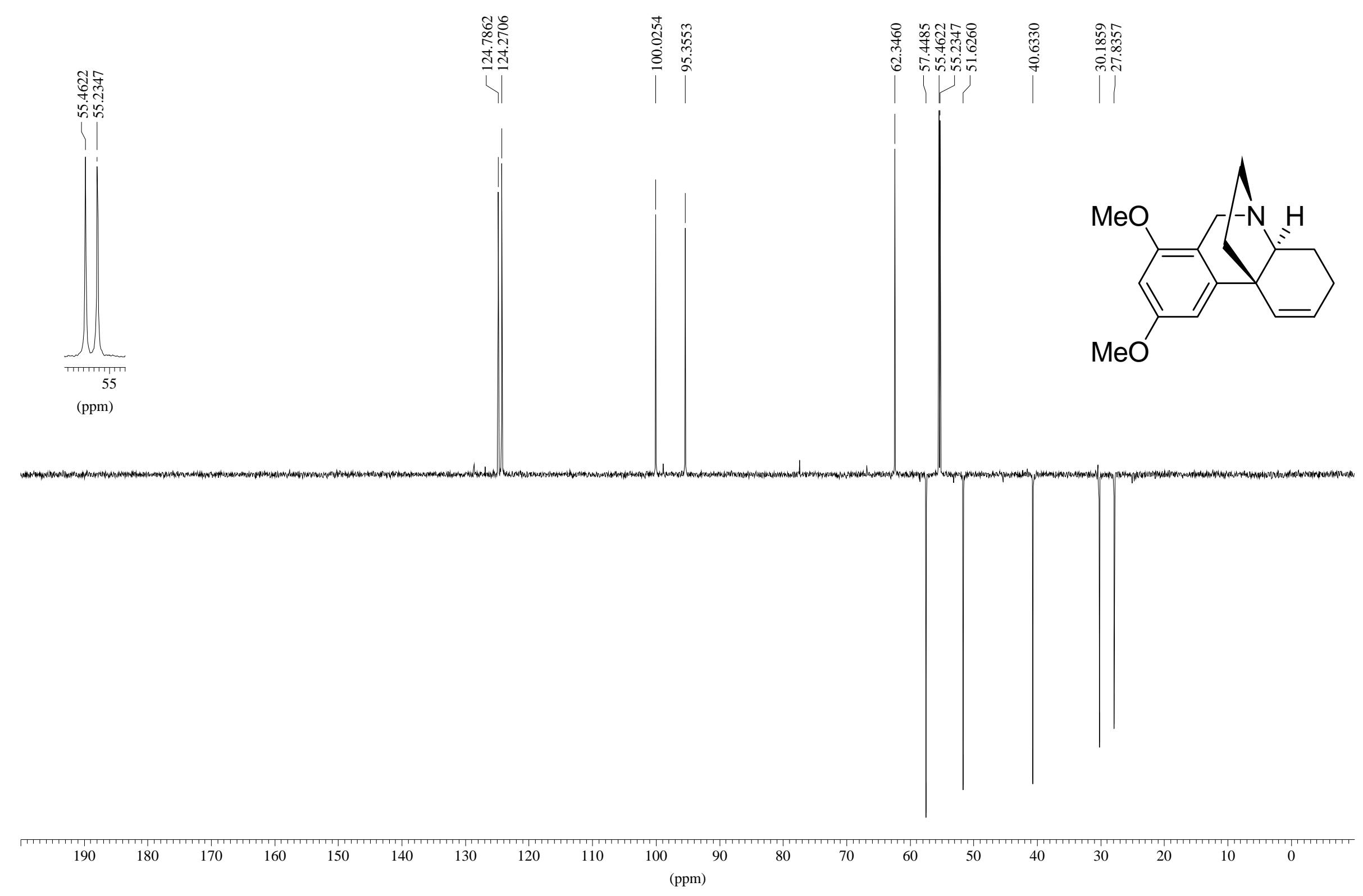

